hep-th/0204035

\title{
Lectures on Supergravity
}

\author{
Friedemann Brandt \\ Max-Planck-Institute for Mathematics in the Sciences, \\ Inselstraße 22-26, D-04103 Leipzig, Germany \\ Published in Fortschr. Phys. 50 (2002) 1126-1172
}

\begin{abstract}
The text is an essentially self-contained introduction to four-dimensional $\mathrm{N}=1$ supergravity, including its couplings to super Yang-Mills and chiral matter multiplets, for readers with basic knowledge of standard gauge theories and general relativity. Emphasis is put on showing how supergravity fits in the general framework of gauge theories and how it can be derived from a tensor calculus for gauge theories of a standard form.
\end{abstract}

\section{Contents}

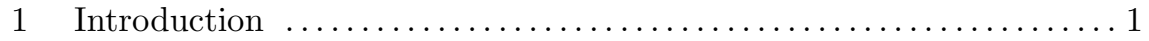

2 Gauge symmetries in the jet space approach $\ldots \ldots \ldots \ldots \ldots \ldots \ldots \ldots$

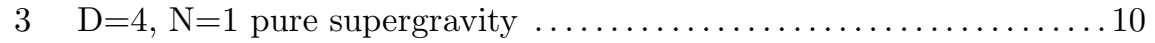

4 Tensor calculus for standard gauge theories $\ldots \ldots \ldots \ldots \ldots \ldots \ldots \ldots$ 16

5 Off-shell formulations of $\mathrm{D}=4, \mathrm{~N}=1$ supergravity with matter .....23

A Lorentz algebra, spinors, Grassmann parity .............. 37

B Explicit verification of local supersymmetry $\ldots \ldots \ldots \ldots \ldots \ldots \ldots 2$

\section{Introduction}

Supergravity (SUGRA) is for several reasons an interesting concept in modern high energy physics. It raises supersymmetry (SUSY) to a gauge symmetry and thus combines two principles of major interest, namely gauge invariance which underlies our present models of fundamental interactions, and SUSY, one of the most promising theoretical concepts for extending these models. In addition SUGRA includes and extends general relativity (GR) which makes it an interesting framework for describing gravitational interactions in high energy physics. In particular SUGRA theories arise in string theory, one of the presently most favoured approaches in the field of "quantum gravity".

SUGRA had an important impact on the development of general concepts in the field of gauge theories, such as the reformulation and refinement of the BRST-approach, because it exhibited properties which are not encountered in more familiar gauge theories, such as Yang-Mills (YM) theories or standard GR. Such properties are gauge transformations whose commutator algebra does not close off-shell or involves field dependent structure functions. Therefore SUGRA can serve as an instructive example to illustrate the general structure of gauge theories.

As SUGRA is a generalization of GR, it may be worthwhile to compile further differences from standard GR. The most fundamental difference is that SUGRA theories 
have more gauge symmetries than standard GR. In particular they have of course local SUSY and contain corresponding gauge fields, so-called gravitinos (Rarita-Schwinger fields) which are spinor fields. Owing to the presence of spinor fields, SUGRA theories are formulated in the vielbein formulation (Cartan formulation) of GR rather than in the metric formulation, and therefore they are always invariant under local Lorentz transformations. Many SUGRA theories, especially in higher dimensions, contain in addition $p$-form gauge fields which generalize the electromagnetic gauge potential and are invariant under corresponding gauge transformations of these fields.

Fields which occur typically in SUGRA theories are thus the vielbein which we denote by $e_{\mu}^{a}$ ( $\mu$ is a "world index" of the same as type as the indices of the metric in GR, $a$ is a Lorentz vector index), the gravitino(s) $\psi_{\mu}$ (whose spinor index has been suppressed), $p$-form gauge fields $A_{\mu_{1} \ldots \mu_{p}}$ which are totally antisymmetric in their world indices (the electromagnetic gauge field and YM gauge fields are 1-form gauge fields in this terminology), and standard matter fields such as spacetime scalar fields $\phi$ or ordinary spinor fields $\lambda$ (again the spinor index has been suppressed). A standard SUGRA theory contains always the vielbein and at least one gravitino. Whether and which other fields are present depends on the particular SUGRA theory.

An important restriction on the possible field content is that the number of bosonic degrees of freedom and the number of fermionic degrees of freedom must coincide onshell (for SUGRA theories of standard type). This is required by SUSY because SUSY relates bosonic fields and fermionic fields in a particular way. The number of degrees of freedom (DOF) relevant here is the number of linearly independent plane wave solutions of the free field equations with given Fourier-momentum, up to linearized gauge transformations. The free field equations are the linearized equations of motion (EOM) in a flat gravitational background. For the standard fields, with standard free field equations, these DOF are compiled in (1.1) and (1.2) where $D$ is the spacetime dimension and $f$ is the real dimension of the smallest nontrivial irreducible spinor representation (for Minkowski signature of the metric). Details of the derivation of the numbers in (1.1) and (1.2) can be found, for example, in [1] and [2], some elementary facts about spinors in various dimensions are provided in appendix $\mathrm{A}$.

\begin{tabular}{c|c|c} 
field & DOF off-shell & DOF on-shell $(D \geq 3)$ \\
\hline vielbein $e_{\mu}^{a}$ & $D(D-1) / 2$ & $D(D-3) / 2$ \\
gravitino $\psi_{\mu}$ & $f(D-1)$ & $f(D-3) / 2$ \\
$p$-form gauge field $A_{\mu_{1} \ldots \mu_{p}}$ & $\left(\begin{array}{c}D-1 \\
p\end{array}\right)$ & $\left(\begin{array}{c}D-2 \\
p\end{array}\right)$ \\
real scalar field $\phi$ & 1 & 1 \\
spinor field $\lambda$ & $f$ & $f / 2$
\end{tabular}

$f= \begin{cases}2^{D / 2-1} \text { for } D \bmod 8=2 & \text { Majorana-Weyl spinors } \\ 2^{\lfloor/ 2\rfloor} \text { for } D \bmod 8=0,1,3,4,6 & \text { Weyl or Majorana spinors* } \\ 2^{(D+1) / 2} \text { for } D \bmod 8=5,7 & \text { Dirac spinors }\end{cases}$

${ }^{*}$ Weyl spinors only if $D=2 k$, no Majorana spinors if $D \bmod 8=6$.

(1.1) contains also the number of DOF off-shell given by the number of independent 
components of the respective field up to gauge transformations (taking reducibility relations into account, if any). These numbers are relevant for so-called off-shell formulations of SUGRA theories, i.e., formulations where the commutator algebra of the gauge transformations closes off-shell. Namely in such formulations the number of bosonic DOF and the number of fermionic DOF must coincide both on-shell and off-shell (again, for SUGRA theories of standard type).

An additional restriction on possible SUGRA theories of standard type is the upper bound on the number of real SUSYs. This upper bound is 32 when one requires that dimensional reduction to $D=4$ must not yield fields with spin $\geq 5 / 2$ (this requirement reflects that theories with spin $\geq 5 / 2$ are believed to be inconsistent or physically unacceptable). The number of SUSYs is often given in terms of the number $N$ of sets of SUSYs where each set has $f$ elements with $f$ as in (1.2) [i.e., the corresponding gauge parameters sit in an irreducible spinor representation]. Hence, if in this terminology one says that a theory has $N$ SUSYs, it has thus actually $N f$ real SUSYs. The bound of at most 32 SUSYs limits standard SUGRA theories, which can be characterized in this way by a value of $N$, to spacetime dimensions $D \leq 11$ because for $D \geq 12$ one has $f \geq 641$ Therefore, SUGRA theories of standard type exist only up to eleven dimensions.

In (1.3) the values of $f$ and the on-shell DOF for some fields are spelled out explicitly for $4 \leq D \leq 11$. In addition the maximal value $N_{\max }$ of $N$ is given. One sees from these numbers, for example, that in $D=4$ it might be possible to construct an $N=1$ SUGRA theory whose only fields are the vielbein $e_{\mu}^{a}$ (called "vierbein" in $D=4$ ) and one gravitino $\psi_{\mu}$ ( $N$ is also the number of gravitinos because these sit in spinor representations with dimension $f$ ). This theory does indeed exist and will be presented in some detail later. Other possibilities would be, for example, a $D=4, N=2$ SUGRA theory with vierbein $e_{\mu}^{a}$, two gravitinos $\psi_{\mu}, \psi_{\mu}^{\prime}$, and one "photon" $A_{\mu}$, or a $D=11, N=1$ SUGRA theory with "elfbein" $e_{\mu}^{a}$, one gravitino $\psi_{\mu}$ and one 3 -form gauge field $A_{\mu \nu \rho}$. Both SUGRA theories do also exist.

\begin{tabular}{c|c|c|c|c|c|c|c|c}
$D$ & 4 & 5 & 6 & 7 & 8 & 9 & 10 & 11 \\
\hline \hline$f$ & 4 & 8 & 8 & 16 & 16 & 16 & 16 & 32 \\
\hline$N_{\max }$ & 8 & 4 & 4 & 2 & 2 & 2 & 2 & 1 \\
\hline DOF of $\psi_{\mu}$ on-shell & 2 & 8 & 12 & 32 & 40 & 48 & 56 & 128 \\
\hline DOF of $e_{\mu}^{a}$ on-shell & 2 & 5 & 9 & 14 & 20 & 27 & 35 & 44 \\
\hline DOF of $A_{\mu}$ on-shell & 2 & 3 & 4 & 5 & 6 & 7 & 8 & 9 \\
\hline DOF of $A_{\mu \nu}$ on-shell & 1 & 3 & 6 & 10 & 15 & 21 & 28 & 36 \\
\hline DOF of $A_{\mu \nu \rho}$ on-shell & 0 & 1 & 4 & 10 & 20 & 35 & 56 & 84
\end{tabular}

The remainder of this text is devoted to $D=4, N=1$ SUGRA. It aims at giving an essentially self-contained introduction to the structure of this theory at a non-expert level, for readers with basic knowledge of GR and standard gauge theories (in particular, some knowledge of YM theory might be helpful). The text is limited to basic material. In particular it does not cover more technical stuff, such as the use of superspace techniques,

\footnotetext{
${ }^{1}$ In "non-standard theories" the number of SUSYs need not be an integer multiple of $f$.
} 
or a discussion of phenomenological aspects, which may be found in textbooks or reviews on SUGRA, such as [3, 4, 5, 6, 7, 8, 1, 9. Rather I have tried to emphasize that and how SUGRA fits in with general principles of gauge theories. These principles are briefly reviewed in section 2. Section 3 presents in some detail the simplest $D=4, N=1$ SUGRA theory mentioned above, whose only fields are the vierbein and the gravitino. Section 4 introduces the concept of a tensor calculus for a class of standard gauge theories. This calculus is used in section 5 as a framework to present the "old minimal" and the "new minimal" off-shell formulations of $D=4, N=1$ SUGRA including the coupling to matter multiplets (super YM multiplets, chiral multiplets). Conventions, especially concerning spinors, and the explicit verification that the SUGRA actions given in the text are indeed supersymmetric are relegated to the appendix.

\section{Gauge symmetries in the jet space approach}

This section assembles the general definition and basic properties of Lagrangean gauge theories, using the jet space approach.

\section{$2.1 \quad$ Jet spaces}

The concept of jet-spaces originates from the theory of partial differential equations (see, for example, [10, 11, 12, 13, 14]). It provides a mathematically rigorous, simple and general framework for the discussion of many aspects of symmetries. For our purposes it suffices to know that jet spaces are spaces whose coordinates are the ordinary coordinates $x^{\mu}$ of a base space $M$ (in our case: spacetime), and additional variables $\partial_{\mu_{1} \ldots \mu_{k}} \phi^{i}$ representing fields $\phi^{i}(k=0)$ and their first and higher order derivatives $(k=1,2, \ldots) 2$. The fields and their derivatives are thus regarded as algebraic objects. The conception of fields as functions of the coordinates $x^{\mu}$, or as mappings from $M$ to some space $F$ arise only as sections $s$ of the corresponding jet bundle over $M$ where the jet variables $\partial_{\mu_{1} \ldots \mu_{k}} \phi^{i}$ turn into functions $M \rightarrow F$ according to

$$
\left.\partial_{\mu_{1} \ldots \mu_{k}} \phi^{i}\right|_{s}=\frac{\partial^{k} \phi^{i}(x)}{\partial x^{\mu_{1}} \ldots \partial x^{\mu_{k}}} .
$$

As the partial derivatives commute on smooth functions, we identify jet coordinates $\partial_{\mu_{1} \ldots \mu_{k}} \phi^{i}$ which differ only by permutations of the derivative indices:

$$
\forall r, s: \quad \partial_{\mu_{1} \ldots \mu_{r} \ldots \mu_{s} \ldots \mu_{k}} \phi^{i}=\partial_{\mu_{1} \ldots \mu_{s} \ldots \mu_{r} \ldots \mu_{k}} \phi^{i} .
$$

One may either work with a set of independent jet coordinates, such as $\left\{x^{\mu}, \partial_{\mu_{1} \ldots \mu_{k}} \phi^{i}\right.$ : $\left.\mu_{i} \leq \mu_{i+1}, k=0,1, \ldots\right\}$, or with the redundant set of all jet variables. We prefer the second option because it allows one to avoid inconvenient combinatorical factors. The partial derivatives are then represented in the infinite jet space by algebraic operations $\partial_{\mu}$ defined by

$$
\partial_{\mu}=\frac{\partial}{\partial x^{\mu}}+\sum_{k \geq 0} \partial_{\mu \mu_{1} \ldots \mu_{k}} \phi^{i} \frac{\partial^{S}}{\partial \partial_{\mu_{1} \ldots \mu_{k}} \phi^{i}},
$$

\footnotetext{
${ }^{2}$ Henceforth we shall work in the infinite jet space, containing all derivatives.
} 
where the derivatives $\partial^{S} / \partial \partial_{\mu_{1} \ldots \mu_{k}} \phi^{i}$ act on the jet variables according to

$$
\frac{\partial^{S} \partial_{\nu_{1} \ldots \nu_{k}} \phi^{j}}{\partial \partial_{\mu_{1} \ldots \mu_{k}} \phi^{i}}=\delta_{i}^{j} \delta_{\left(\nu_{1}\right.}^{\mu_{1}} \ldots \delta_{\left.\nu_{k}\right)}^{\mu_{k}}, \quad k \neq r: \frac{\partial^{S} \partial_{\nu_{1} \ldots \nu_{r}} \phi^{j}}{\partial \partial_{\mu_{1} \ldots \mu_{k}} \phi^{i}}=0 .
$$

Here the round parantheses denote symmetrization with weight one, such as $\delta_{(\mu}^{\rho} \delta_{\nu)}^{\sigma}=$ $1 / 2\left(\delta_{\mu}^{\rho} \delta_{\nu}^{\sigma}+\delta_{\nu}^{\rho} \delta_{\mu}^{\sigma}\right)$. With these definitions the derivatives $\partial_{\mu}$ have indeed the same algebraic properties as the partial derivatives of smooth functions - as they should, in order that (2.1) makes sense. In particular they satisfy

$$
\partial_{\mu} \partial_{\mu_{1} \ldots \mu_{k}} \phi^{i}=\partial_{\mu \mu_{1} \ldots \mu_{k}} \phi^{i}
$$

and they commute,

$$
\left[\partial_{\mu}, \partial_{\nu}\right]=0
$$

A basic and important fact is that a function $f$ on the jet space is a total divergence if and only if it has vanishing Euler-Lagrange derivatives with respect to all fields on which it depends,

$$
f(x,[\phi])=\partial_{\mu} K^{\mu}(x,[\phi]) \quad \Leftrightarrow \quad \frac{\hat{\partial} f(x,[\phi])}{\hat{\partial} \phi^{i}}=0 \quad \forall \phi^{i},
$$

where $\hat{\partial} f / \hat{\partial} \phi^{i}$ denotes the Euler-Lagrange derivative of $f$ with respect to $\phi^{i}$,

$$
\frac{\hat{\partial} f(x,[\phi])}{\hat{\partial} \phi^{i}}=\sum_{k \geq 0}(-)^{k} \partial_{\mu_{1}} \ldots \partial_{\mu_{k}} \frac{\partial^{S} f(x,[\phi])}{\partial \partial_{\mu_{1} \ldots \mu_{k}} \phi^{i}} .
$$

Here $[\phi]$ indicates local dependence on the fields (which usually means dependence on derivatives up to some arbitrary but finite order).

\subsection{Gauge symmetries of a Lagrangian}

An (infinitesimal) gauge transformation is a transformation of the fields involving linearly "gauge parameters" or their derivatives. The gauge parameters are arbitrary fields and therefore these parameters and their derivatives are also treated as jet variables. Hence, when dealing with gauge symmetries, we work in an enlarged jet space involving also these extra variables in addition to the coordinates $x^{\mu}$ and the "fields" $\phi^{i}$ and their derivatives. The basic difference between the gauge parameters and the fields is that the former do not occur in the Lagrangian and the field equations (Euler-Lagrange equations of motion) derived from it. Hence the Lagrangian is a function on the "original" jet space with coordinates $x^{\mu}$ and $\partial_{\mu_{1} \ldots \mu_{k}} \phi^{i}$. Each jet variable (including the gauge parameters and their derivatives) has a Grassmann parity which is 0 for "bosonic" (commuting) fields or 1 for "fermionic" (anticommuting) fields, cf. appendix A for our conventions in the SUGRA context. By assumption, the Lagrangian is a Grassmann even function on the jet space and the gauge transformations are Grassmann even operations. 
Gauge transformations $\delta_{\xi} \phi^{i}$ of the fields are given by operators $R_{M}^{i}$ which act on gauge parameters $\xi^{M}$ and may depend on the fields and their derivatives:

$$
\delta_{\xi} \phi^{i}=R_{M}^{i} \xi^{M}, \quad R_{M}^{i}=\sum_{k=0}^{m} r_{M}^{i \mu_{1} \ldots \mu_{k}}(x,[\phi]) \partial_{\mu_{1}} \ldots \partial_{\mu_{k}} .
$$

These transformations are extended to derivatives of the fields and to local functions on jet spaces according to

$$
\delta_{\xi}=\sum_{k \geq 0}\left(\partial_{\mu_{1}} \ldots \partial_{\mu_{k}} \delta_{\xi} \phi^{i}\right) \frac{\partial^{S}}{\partial \partial_{\mu_{1} \ldots \mu_{k}} \phi^{i}} .
$$

In particular this gives

$$
\begin{gathered}
{\left[\delta_{\xi}, \partial_{\mu}\right]=0,} \\
\delta_{\xi} x^{\mu}=0, \\
\delta_{\xi}(a b)=\left(\delta_{\xi} a\right) b+a\left(\delta_{\xi} b\right) .
\end{gathered}
$$

(2.11) means simply that the gauge transformations of the derivatives of the fields are so-called 'prolongations' of the gauge transformations of the undifferentiated fields $\left(\delta_{\xi} \partial_{\mu} \phi^{i}=\partial_{\mu}\left(\delta_{\xi} \phi^{i}\right)\right.$ etc). (2.12) means that explicit coordinates $x^{\mu}$ are never transformed, i.e., when evaluated on a section of the jet bundle, $\left(\delta_{\xi} \phi^{i}\right)(x)$ represents the transformation of $\phi^{i}(x)$ as a function of its arguments but not of the arguments themselves (it represents thus the Lie derivative of $\phi^{i}$ ). (2.13) is the Leibniz rule and means that the gauge transformations are derivations on the jet space. We can now define gauge symmetries of a Lagrangian:

Definition. A gauge transformation $\delta_{\xi}$ is called a gauge symmetry of a Lagrangian $L(x,[\phi])$ if it leaves the Lagrangian invariant up to a total divergence:

$$
\delta_{\xi} L(x,[\phi])=\partial_{\mu} K^{\mu}(x,[\phi, \xi]) .
$$

\subsection{Noether identities and gauge symmetry of the EOM}

Owing to (2.7), the gauge invariance condition (2.14) imposes

$$
\frac{\hat{\partial} \delta_{\xi} L(x,[\phi])}{\hat{\partial} \xi^{M}}=0 \quad \forall \xi^{M}
$$

and

$$
\frac{\hat{\partial} \delta_{\xi} L(x,[\phi])}{\hat{\partial} \phi^{i}}=0 \quad \forall \phi^{i} .
$$

(2.15) are the Noether identities corresponding to the gauge symmetry. Explicitly they read

$$
(-)^{\left|\phi^{i}\right|} R_{M}^{i+} \frac{\hat{\partial} L(x,[\phi])}{\hat{\partial} \phi^{i}}=0
$$


where $\left|\phi^{i}\right|$ is the Grassmann parity of $\phi^{i}$ and $R_{M}^{i+}$ is the operator adjoint to the operator $R_{M}^{i}$ which defines the gauge symmetry according to (2.9). This adjoint operator is given, on all functions $f$ on the jet space, by

$$
R_{M}^{i+} f=\sum_{k \geq 0}(-)^{k} \partial_{\mu_{1}} \ldots \partial_{\mu_{k}}\left[f r_{M}^{i \mu_{1} \ldots \mu_{k}}(x,[\phi])\right] .
$$

(2.16) yields the gauge transformations of the EOM. Explicitly one obtains (see, for example, formula (6.43) of [15]):

$$
\delta_{\xi} \frac{\hat{\partial} L(x,[\phi])}{\hat{\partial} \phi^{i}}=-\sum_{k \geq 0}(-)^{k} \partial_{\mu_{1}} \ldots \partial_{\mu_{k}}\left[\frac{\partial^{S} \delta_{\xi} \phi^{j}}{\partial \partial_{\mu_{1} \ldots \mu_{k}} \phi^{i}} \frac{\hat{\partial} L(x,[\phi])}{\hat{\partial} \phi^{j}}\right] .
$$

Remark. Actually (2.17) is equivalent to (2.14) (Noether's second theorem [16]). The reason is that every term in $\delta_{\xi} L$ is linear in the $\xi$ 's (or their derivatives) which implies

$$
\delta_{\xi} L(x,[\phi])=\xi^{M} \frac{\hat{\partial} \delta_{\xi} L(x,[\phi])}{\hat{\partial} \xi^{M}}+\partial_{\mu} K^{\mu}(x,[\phi, \xi]) .
$$

Hence (2.17) implies indeed (2.14), and thus it also implies (2.16).

\subsection{Trivial gauge symmetries}

Consider the transformations

$$
\delta^{\text {triv }} \phi^{i}=\sum_{k, m \geq 0}(-)^{k} \partial_{\mu_{1}} \ldots \partial_{\mu_{k}}\left[M^{j\left(\nu_{1} \ldots \nu_{m}\right) i\left(\mu_{1} \ldots \mu_{k}\right)}(x,[\phi, \xi]) \partial_{\nu_{1}} \ldots \partial_{\nu_{m}} \frac{\hat{\partial} L}{\hat{\partial} \phi^{j}}\right]
$$

where

$$
M^{j\left(\nu_{1} \ldots \nu_{m}\right) i\left(\mu_{1} \ldots \mu_{k}\right)}(x,[\phi, \xi])=-(-)^{\left|\phi^{i}\right|\left|\phi^{j}\right|} M^{i\left(\mu_{1} \ldots \mu_{k}\right) j\left(\nu_{1} \ldots \nu_{m}\right)}(x,[\phi, \xi]) .
$$

The transformation (2.20), extended to the whole jet space as in (2.10), is a gauge symmetry of $L$ according to our definition because of

$$
\delta^{\text {triv }} L \sim M^{j\left(\nu_{1} \ldots \nu_{m}\right) i\left(\mu_{1} \ldots \mu_{k}\right)}(x,[\phi, \xi])\left[\partial_{\mu_{1}} \ldots \partial_{\mu_{k}} \frac{\hat{\partial} L}{\hat{\partial} \phi^{i}}\right]\left[\partial_{\nu_{1}} \ldots \partial_{\nu_{m}} \frac{\hat{\partial} L}{\hat{\partial} \phi^{j}}\right]=0
$$

where $\sim$ denotes equality up to a total divergence and the last equality $(=0)$ holds because of the graded antisymmetry of the $M$ 's as in (2.21). For obvious reasons, such transformations are called trivial gauge symmetries. They exist for every Lagrangian and vanish on-shell, i.e., they vanish on all solutions of the EOM. Conversely one can prove under fairly general assumptions (regularity conditions) that a gauge symmetry which vanishes on-shell takes necessarily the form (2.20) [17, 15]. 


\subsection{Generating set of gauge symmetries}

When trying to characterize the gauge symmetries of a model satisfactorily one faces two complications. On the one hand, one has to deal with the trivial gauge symmetries which one wants to "mod out". On the other hand one has to take the following fact into account: whenever $\delta_{\xi} \phi^{i}=R_{M}^{i} \xi^{M}$ is a gauge symmetry, then $\hat{\delta}_{\hat{\xi}} \phi^{i}=\hat{R}_{A}^{i} \hat{\xi}^{A}$ with $\hat{R}_{A}^{i}=R_{M}^{i} K_{A}^{M}$ is also a gauge symmetry, for any (possibly field dependent) local operators $K_{A}^{M}$ : indeed, when $\delta_{\xi}$ is a gauge symmetry, it satisfies (2.14) for all $\xi$ 's and thus in particular for $\xi^{M}=K_{A}^{M} \hat{\xi}^{A}$, whatever operators $K_{A}^{M}$ we choose and for arbitrary $\hat{\xi}^{A}$. Notice that even the range of the index $A$ may differ from the range of the index $M$. But clearly $\hat{\delta}_{\hat{\xi}}$ is not a new gauge symmetry as it arises from $\delta_{\xi}$ just by substituting $f^{M}(x,[\phi, \hat{\xi}])=K_{A}^{M} \hat{\xi}^{A}$ for $\xi^{M}$. This motivates the following definition: we say a set of operators $\left\{R_{M}^{i}\right\}$ provides a generating set of the gauge symmetries of a Lagrangian if any gauge symmetry of the Lagrangian can be generated through them according to

$$
\delta_{\xi} L=\partial_{\mu} K^{\mu} \Rightarrow \delta_{\xi} \phi^{i}=R_{M}^{i} f^{M}(x,[\phi, \xi])+\delta^{\text {triv }} \phi^{i},
$$

for some local functions $f^{M}(x,[\phi, \xi])$.

The concept of a generating set of gauge symmetries is of fundamental importance for the theory of gauge symmetries. It is somewhat analogous to the concept of a basis of a vector space although the analogy must be used with great care because a generating set evidently is not a basis of gauge symmetries in the vector space sense. Within the analogy, (2.22) corresponds to the completeness of a basis of a vector space. The independence of the elements of a basis also has a counterpart: it is the irreducibility of a generating set. The latter requires that the operators $R_{M}^{i}$ have no nontrivial 'zero mode', i.e.,

$$
R_{M}^{i} f^{M}(x,[\phi, \xi])=\delta^{\text {triv }} \phi^{i} \Rightarrow f^{M}(x,[\phi, \xi]) \approx 0 \quad \text { (irreducibility) }
$$

where $\approx$ is equality on-shell,

$$
f \approx g \quad: \Leftrightarrow \quad f-g=M^{i} \frac{\hat{\partial} L(x,[\phi])}{\hat{\partial} \phi^{i}}
$$

for some local operators $M^{i}$. However, unlike the situation in the case of (finite dimensional) vector spaces, it is not always possible to choose an irreducible set because locality may obstruct this. So, one sometimes has to deal with reducible generating sets of gauge transformations.

The choice of a generating set of gauge transformations is by no means unique; switching from one generating set to another one corresponds in the above analogy to changing the basis of a vecor space, albeit the freedom in the choice of a generating set evidently exceeds by far the freedom in the choice of a basis of a vector space. The relation between two generating sets $\left\{R_{M}^{i}\right\}$ and $\left\{\hat{R}_{A}^{i}\right\}$ is of the type discussed above,

$$
\hat{R}_{A}^{i} \approx R_{M}^{i} K_{A}^{M}, \quad R_{M}^{i} \approx \hat{R}_{A}^{i} \hat{K}_{M}^{A},
$$

for some local, generally field dependent operators $K_{A}^{M}$ and $\hat{K}_{M}^{A}$. Again, the ranges of the indices $M$ and $A$ may differ; in particular one may switch from an irreducible to a 
reducible set. Notice that switching between different generating sets is accompanied by redefinitions of the corresponding sets of gauge parameters because (2.25) yields

$$
\begin{array}{ll}
\hat{\delta}_{\hat{\xi}}=\delta_{K \hat{\xi}}+\delta^{\text {triv }}, & (K \hat{\xi})^{M}=K_{A}^{M} \hat{\xi}^{A}, \\
\delta_{\xi}=\hat{\delta}_{\hat{K} \xi}+\delta^{\text {triv }}, & (\hat{K} \xi)^{A}=\hat{K}_{M}^{A} \xi^{M} .
\end{array}
$$

Example. Let us consider 3-dimensional abelian Chern-Simons theory with Lagrangian

$$
L=\epsilon^{\mu \nu \rho} A_{\mu} \partial_{\nu} A_{\rho}
$$

The set of fields $\phi^{i}$ is in this case given by the components of the gauge field,

$$
\left\{\phi^{i}\right\} \equiv\left\{A_{\mu}\right\} .
$$

It can be proved that a generating set of gauge symmetries of the abelian Chern-Simons Lagrangian is given by the abelian gauge transformations

$$
\delta_{\xi} A_{\mu}=\partial_{\mu} \xi
$$

The corresponding set of operators $R_{N}^{i}$ is thus given just by the derivatives $\partial_{\mu}$ :

$$
\left\{R_{N}^{i}\right\} \equiv\left\{\partial_{\mu}\right\}
$$

Now, if this provides really a generating set, it must be possible to express every gauge symmetry of the abelian Chern-Simons Lagrangian in terms of these operators up to trivial gauge symmetries, as in (2.22). Let us verify that this holds for the spacetime diffeomorphisms [the latter are indeed gauge symmetries because the Chern-Simons Lagrangian is a scalar density with weight one under spacetime diffeomorphisms]:

$$
\begin{aligned}
\delta_{\text {diffeo }} A_{\mu} & =\xi^{\nu} \partial_{\nu} A_{\mu}+\partial_{\mu} \xi^{\nu} A_{\nu} \\
& =\xi^{\nu}\left(\partial_{\nu} A_{\mu}-\partial_{\mu} A_{\nu}\right)+\xi^{\nu} \partial_{\mu} A_{\nu}+\partial_{\mu} \xi^{\nu} A_{\nu} \\
& =\frac{1}{2} \xi^{\nu} \epsilon_{\nu \mu \rho} \frac{\hat{\partial} L}{\hat{\partial} A_{\rho}}+\partial_{\mu}\left(\xi^{\nu} A_{\nu}\right) .
\end{aligned}
$$

Note that the first term in the last line is a trivial symmetry as in (2.20) (with $M^{j i} \equiv$ $\frac{1}{2} \xi^{\nu} \epsilon_{\nu \mu \rho}$ ), while the second term is of the form $R_{M}^{i} f^{M}(\xi, \phi)$ (with $f^{M} \equiv \xi^{\nu} A_{\nu}$ ).

\subsection{Algebra of gauge symmetries}

The concept of a generating set of gauge symmetries allows one to derive the general form of the commutator algebra of gauge symmetries. The commutator of two gauge symmetries $\delta_{\xi_{1}}$ and $\delta_{\xi_{2}}$ is again a gauge symmetry because it leaves the Lagrangian invariant (simply because $\delta_{\xi} L=\partial_{\mu} K^{\mu}(x,[\phi, \xi])$ and $\left[\delta_{\xi}, \partial_{\mu}\right]=0$ imply $\left.\delta_{\xi_{1}} \delta_{\xi_{2}} L=\delta_{\xi_{1}} \partial_{\mu} K^{\mu}\left(x,\left[\phi, \xi_{2}\right]\right)=\partial_{\mu} \delta_{\xi_{1}} K^{\mu}\left(x,\left[\phi, \xi_{2}\right]\right)\right)$ and is a derivation (because the commutator of two derivations is again a derivation). Owing to (2.22) it can thus be 
expressed through the operators $R_{M}^{i}$ of the generating set and a trivial gauge symmetry. In particular one has

$$
\delta_{\xi_{1}} \phi^{i}=R_{M}^{i} \xi_{1}^{M}, \delta_{\xi_{2}} \phi^{i}=R_{M}^{i} \xi_{2}^{M} \Rightarrow\left[\delta_{\xi_{1}}, \delta_{\xi_{2}}\right] \phi^{i}=R_{M}^{i} f^{M}\left(x,\left[\phi, \xi_{1}, \xi_{2}\right]\right)+\delta^{\text {triv }} \phi^{i}
$$

Notice that $R_{M}^{i} f^{M}$ is a gauge transformation $\delta_{f}$ as $\delta_{\xi_{1}} \phi^{i}$ and $\delta_{\xi_{2}} \phi^{i}$ but with "composite" (possibly field dependent) parameter $f^{M}\left(x,\left[\phi, \xi_{1}, \xi_{2}\right]\right)$. Owing to (2.19), the commutator of a trivial gauge symmetry and any other gauge symmetry (trivial or non-trivial) vanishes on-shell,

$$
\left[\delta^{\text {triv }}, \delta_{\xi}\right] \phi^{i} \approx 0 \quad \forall \phi^{i} .
$$

As already remarked at the end of section 2.4 this implies under fairly general assumptions that this commutator is again a trivial gauge symmetry,

$$
\left[\delta^{\text {triv }}, \delta_{\xi}\right]=\tilde{\delta}^{\text {triv }}
$$

Hence the only possibly nontrivial part of the commutator algebra of gauge symmetries is made up of the terms $R_{M}^{i} f^{M}\left(x,\left[\phi, \xi_{1}, \xi_{2}\right]\right)$ in the commutators of two nontrivial gauge symmetries as in (2.27). If these commutators involve a nonvanishing $\delta^{\text {triv }}$ on the right hand side, the commutator algebra is called an "open gauge algebra". Notice that it may depend on the choice of the generating set whether or not the algebra is open.

\section{$3 \mathrm{D}=4, \mathrm{~N}=1$ pure SUGRA}

This section presents the Lagrangian and gauge transformations of the simplest fourdimensional SUGRA theory [18, 19] $(N=1$ SUGRA without matter multiplets $)$ in the basic formulation with open gauge algebra, using the Weyl-spinor notation as in appendix $\mathrm{A}$.

\subsection{Lagrangian}

Vielbein formulation. Owing to the presence of spinor fields, SUGRA theories are constructed in the vielbein formulation (Cartan formulation) of general relativity. In $D$ dimensions the vielbein is a real $D \times D$-matrix field denoted by $e_{\mu}^{a}$ and related to the spacetime metric $g_{\mu \nu}$ according to

$$
g_{\mu \nu}=e_{\mu}^{a} e_{\nu}^{b} \eta_{a b}
$$

where $\eta_{a b}$ is the Minkowski metric. In order that the metric be invertible, the vielbein must be invertible. We denote its inverse by $E_{a}^{\mu}$,

$$
e_{\mu}^{a} E_{b}^{\mu}=\delta_{b}^{a}, \quad e_{\mu}^{a} E_{a}^{\nu}=\delta_{\mu}^{\nu} .
$$

In contrast to the standard (metric) formulation of general relativity, the metric is thus not treated as an elementary field but constructed from the vielbein according to (3.1). Conversely, given a metric (with the same signature as the Minkowski metric), one can 
always construct a vielbein satisfying (3.1): as the metric is symmetric, it can at each point be diagonalized by some orthogonal matrix $O$ and one may choose the vielbein as $D O$ where $D=\operatorname{diag}\left(\left|r_{1}\right|^{1 / 2}, \ldots,\left|r_{D}\right|^{1 / 2}\right)$ is a diagonal matrix and the $r$ 's are the eigenvalues of the metric. Of course, both $O$ and the $r$ 's in general depend on the point, i.e., they are fields, and so is the vielbein. Actually this choice of the vielbein is not unique because (3.1) determines the vielbein only modulo arbitrary local Lorentz transformations as these leave the Minkowski metric invariant. Hence GR has in the vielbein formulation more gauge symmetries than in the metric formulation because it is also invariant under local Lorentz transformations in addition to the diffeomorphism invariance.

SUGRA Lagrangian in first order formulation. In four dimensions the vielbein is called vierbein. The gravitino is denoted by $\psi_{\mu}^{\alpha}$ where $\alpha$ are Weyl spinor indices, see appendix A. Hence, for each value of $\mu, \psi_{\mu}^{\alpha}$ is a complex 2-component Weyl spinor field. Its complex conjugate is denoted by $\bar{\psi}_{\mu}^{\dot{\alpha}}$. Our index notation is thus: Greek indices from the beginning of the alphabet denote Weyl spinor indices, Greek indices from the middle of the alphabet denote world indices and lower case Latin indices from the beginning of the alphabet denote Lorentz vector indices. The spinor indices and the Lorentz vector indices indicate the transformation properties under local Lorentz transformations, the world indices the transformation properties under spacetime diffeomorphisms.

In addition to the vielbein and the gravitino one may introduce the so-called spin connection $\omega_{\mu}^{a b}=-\omega_{\mu}^{b a}$ as an independent field. It serves as the gauge field for the local Lorentz transformations. However, it is only an auxiliary field, i.e., it can be eliminated by solving algebraically its EOM. The formulation with the spin connection as an auxiliary field is called first order formulation, the one which uses from the very beginning only the vielbein and the gravitino is called second order formulation. We shall first introduce the first order formulation and then focus on the second order formulation.

In the first order formulation, the Lagrangian is a function of the vielbein, gravitino, spin connection and their derivatives, given by

$$
L=\frac{1}{2} e E_{b}^{\mu} E_{a}^{\nu} R_{\mu \nu}^{a b}+2\left(\nabla_{\mu} \psi_{\nu} \sigma_{\rho} \bar{\psi}_{\sigma}+\psi_{\sigma} \sigma_{\rho} \nabla_{\mu} \bar{\psi}_{\nu}\right) \epsilon^{\mu \nu \rho \sigma}
$$

where:

$$
\begin{aligned}
e & =\operatorname{det}\left(e_{\mu}^{a}\right), \\
R_{\mu \nu}^{a b} & \left.=\partial_{\mu} \omega_{\nu}^{a b}-\partial_{\nu} \omega_{\mu}^{a b}+\omega_{\mu}^{a c} \omega_{\nu c}^{b}-\omega_{\nu}^{a c} \omega_{\mu c}^{b} \quad \text { (field strength of } \omega_{\mu}^{a b}\right), \\
\nabla_{\mu} \psi_{\nu}^{\alpha} & =\partial_{\mu} \psi_{\nu}^{\alpha}-\frac{1}{2} \omega_{\mu}^{a b}\left(\psi_{\nu} \sigma_{a b}\right)^{\alpha} \quad\left(\text { Lorentz-covariant derivative of } \psi_{\mu}\right), \\
\nabla_{\mu} \bar{\psi}_{\nu}^{\dot{\alpha}} & \left.=\partial_{\mu} \bar{\psi}_{\nu}^{\dot{\alpha}}+\frac{1}{2} \omega_{\mu}^{a b}\left(\bar{\sigma}_{a b} \bar{\psi}_{\nu}\right)^{\dot{\alpha}} \quad \text { (Lorentz-covariant derivative of } \bar{\psi}_{\mu}\right), \\
\sigma_{\rho \alpha \dot{\alpha}} & =e_{\rho}^{a} \sigma_{a \alpha \dot{\alpha}} \quad(\text { field dependent!), } \\
\epsilon^{\mu \nu \rho \sigma} & =e \underbrace{E_{a}^{\mu} E_{b}^{\nu} E_{c}^{\rho} E_{d}^{\sigma} \epsilon^{a b c d}}_{\propto \operatorname{det}\left(E_{a}^{\mu}\right)=1 / e} \in\{0,1,-1\} \quad \text { (field independent!). }
\end{aligned}
$$




\section{Remarks:}

- The Lorentz-covariant derivative $\nabla_{\mu}$ is built in the standard manner (cf. electrodynamics, YM theory, GR, ...) by means of the gauge field $\omega_{\mu}^{a b}$. It is defined not only on spinor fields, but also on any other Lorentz-covariant fields by

$$
\nabla_{\mu}=\partial_{\mu}-\frac{1}{2} \omega_{\mu}^{a b} l_{a b} \quad\left(\text { conventional factor } 1 / 2 \text { because of } l_{a b}=-l_{b a}\right) .
$$

- $E_{b}^{\mu} E_{a}^{\nu} R_{\mu \nu}^{a b}$ is a spacetime curvature scalar built from $R_{\mu \nu}{ }^{a b}$,

$$
R=E_{b}^{\mu} E_{a}^{\nu} R_{\mu \nu}^{a b} \quad \text { ("curvature scalar"). }
$$

- Because of the antisymmetry of $\epsilon^{\mu \nu \rho \sigma}$, the derivatives of $\psi_{\mu}$ and $\bar{\psi}_{\mu}$ occur only through the combinations

$$
\begin{array}{ll}
\nabla_{\mu} \psi_{\nu}-\nabla_{\nu} \psi_{\mu} & \text { ("field strength of } \left.\psi_{\mu} "\right), \\
\nabla_{\mu} \bar{\psi}_{\nu}-\nabla_{\nu} \bar{\psi}_{\mu} & \text { ("field strength of } \left.\bar{\psi}_{\mu} "\right) \text {. }
\end{array}
$$

- In terms of Majorana-spinors $\Psi_{\mu}$ (see appendix $\underline{\mathrm{A}}$ ), one has

$$
\begin{aligned}
\left(\nabla_{\mu} \psi_{\nu} \sigma_{\rho} \bar{\psi}_{\sigma}+\psi_{\sigma} \sigma_{\rho} \nabla_{\mu} \bar{\psi}_{\nu}\right) \epsilon^{\mu \nu \rho \sigma} & =-\bar{\Psi}_{\mu} \hat{\gamma} \gamma_{\nu} \nabla_{\rho} \Psi_{\sigma} \epsilon^{\mu \nu \rho \sigma} \\
& =\mathrm{i} e \bar{\Psi}_{\mu} \gamma^{[\mu} \gamma^{\nu} \gamma^{\rho]} \nabla_{\nu} \Psi_{\rho}, \quad \Psi_{\mu}=\left(\begin{array}{c}
\psi_{\mu \alpha} \\
\bar{\psi}_{\mu}^{\dot{\alpha}}
\end{array}\right) .
\end{aligned}
$$

- Notice that $e= \pm \sqrt{-\operatorname{det}\left(g_{\mu \nu}\right)}$ because of (3.1).

- The definition of $\sigma_{\mu}$ illustrates the general rule how one converts Lorentz-indices into world-indices and vice versa by means of the vierbein and its inverse:

$$
X_{\mu}=e_{\mu}^{a} X_{a}, \quad X_{a}=E_{a}^{\mu} X_{\mu}, \quad X_{\mu \nu}=e_{\mu}^{a} e_{\nu}^{b} X_{a b} \quad \text { etc. }
$$

Determination of $\omega_{\mu}^{a b}$ from its EOM and second order formulation. Varying $\omega_{\mu}^{a b}$ in the Lagrangian (3.3) yields

$$
\begin{aligned}
& L([e, \psi, \omega+\delta \omega])-L([e, \psi, \omega])=\frac{e}{2} E_{b}^{\mu} E_{a}^{\nu}\left(\nabla_{\mu} \delta \omega_{\nu}^{a b}-\nabla_{\nu} \delta \omega_{\mu}^{a b}\right) \\
& -\delta \omega_{\mu}^{a b} \epsilon^{\mu \nu \rho \sigma} \psi_{\nu} \underbrace{\left(\sigma_{a b} \sigma_{\rho}+\sigma_{\rho} \bar{\sigma}_{a b}\right)}_{=\mathrm{i} \epsilon_{a b \rho c} \sigma^{c}} \bar{\psi}_{\sigma} \\
& \Rightarrow \frac{\hat{\partial} L([e, \psi, \omega])}{\hat{\partial} \omega_{\mu}^{a b}}=\frac{1}{2} \nabla_{\nu}\left(e E_{b}^{\mu} E_{a}^{\nu}-e E_{a}^{\mu} E_{b}^{\nu}\right)+6 e i E_{[a}^{\mu} E_{b}^{\nu} E_{c]}^{\sigma} \psi_{\nu} \sigma^{c} \bar{\psi}_{\sigma} \\
& =\frac{1}{2} \partial_{\nu}\left(e E_{b}^{\mu} E_{a}^{\nu}-e E_{a}^{\mu} E_{b}^{\nu}\right)+e \omega_{[a b]}^{\mu}-e \omega_{c}^{c}{ }_{[a} E_{b]}^{\mu}+6 e \mathrm{i} E_{[a}^{\mu} E_{b}^{\nu} E_{c]}^{\sigma} \psi_{\nu} \sigma^{c} \bar{\psi}_{\sigma}
\end{aligned}
$$

where $[. .$.$] denotes complete antisymmetrization with "weight one", and the above rules$ for conversion of world and Lorentz indices were used, e.g.:

$$
\omega_{[a b]}^{\mu}=\frac{1}{2}\left(\omega_{a b}^{\mu}-\omega_{b a}^{\mu}\right), \quad \omega_{a b}^{\mu}=E_{a}^{\nu} \omega_{\nu}^{c d} \eta_{b c} E_{d}^{\mu} .
$$


The EOM for the $\omega_{\mu}^{a b}$ are $\hat{\partial} L / \hat{\partial} \omega_{\mu}^{a b}=0$. They can be solved algebraically for the $\omega_{\mu}^{a b}$ (the $\omega_{\mu}^{a b}$ appear only linearly and undifferentiated in $\hat{\partial} L / \hat{\partial} \omega_{\mu}^{a b}$ ). To do so, one may first determine $\omega_{a b}{ }^{a}$ by contracting the equation $\hat{\partial} L / \hat{\partial} \omega_{\mu}{ }^{a b}=0$ with $e_{\mu}^{a}$, then insert the result into $\hat{\partial} L / \hat{\partial} \omega_{\mu}^{a b}=0$ and solve the latter for $\omega_{[a b]}^{\mu}$ (hint: use the identity $\left.\partial_{\mu} e=e E_{a}^{\nu} \partial_{\mu} e_{\nu}^{a}\right)$. The result is, written in convenient form:

$$
\omega_{[\mu \nu]}^{a}=\partial_{[\mu} e_{\nu]}^{a}-2 \mathrm{i} \psi_{[\mu} \sigma^{a} \bar{\psi}_{\nu]}
$$

This yields $\omega_{\mu}^{a b}$ because $\omega_{\mu \nu \rho}=-\omega_{\mu \rho \nu}$ implies

$$
\omega_{\mu \nu \rho}=\omega_{[\mu \nu] \rho}-\omega_{[\nu \rho] \mu}+\omega_{[\rho \mu] \nu} \Rightarrow \omega_{\mu}^{a b}=\omega_{[\nu \rho]}^{c}\left(2 \delta_{\mu}^{\nu} E^{\rho[a} \delta_{c}^{b]}-e_{\mu c} E^{\nu a} E^{\rho b}\right) .
$$

Using (3.4), we obtain:

$$
\omega_{\mu}^{a b}=E^{\nu a} \partial_{[\mu} e_{\nu]}^{b}-E^{\nu b} \partial_{[\mu} e_{\nu]}^{a}-e_{\mu c} E^{\nu a} E^{\rho b} \partial_{[\nu} e_{\rho]}^{c}+2 \mathrm{i}\left(\psi_{\mu} \sigma^{[a} \bar{\psi}^{b]}+\psi^{[a} \sigma^{b]} \bar{\psi}_{\mu}+\psi^{[a} \sigma_{\mu} \bar{\psi}^{b]}\right) .
$$

The Lagrangian in the second order formulation is given by (3.3) with $\omega_{\mu}^{a b}$ as in (3.5).

\section{$3.2 \quad \mathrm{EOM}$}

From now on we shall always work in the second order formulation, i.e., with $\omega_{\mu}^{a b}$ as in (3.5). The Euler-Lagrange derivatives of the second order Lagrangian (3.3) with respect to the vierbein and gravitino are (one may apply the "1.5 order formalism" here, see appendix B.1):

$$
\begin{aligned}
& \frac{\hat{\partial} L}{\hat{\partial} e_{\mu}^{a}}=e\left(\frac{1}{2} E_{a}^{\mu} R-R_{\rho \nu}{ }^{b c} E_{a}^{\rho} E_{b}^{\nu} E_{c}^{\mu}\right)+2 \epsilon^{\mu \nu \rho \sigma}\left(\nabla_{\nu} \psi_{\rho} \sigma_{a} \bar{\psi}_{\sigma}+\psi_{\sigma} \sigma_{a} \nabla_{\nu} \bar{\psi}_{\rho}\right), \\
& \frac{\hat{\partial} L}{\hat{\partial} \psi_{\mu}^{\alpha}}=-4 \epsilon^{\mu \nu \rho \sigma}\left(\sigma_{\nu} \nabla_{\rho} \bar{\psi}_{\sigma}\right)_{\alpha}, \quad \frac{\hat{\partial} L}{\hat{\partial} \bar{\psi}_{\mu}^{\dot{\alpha}}}=4 \epsilon^{\mu \nu \rho \sigma}\left(\nabla_{\rho} \psi_{\sigma} \sigma_{\nu}\right)_{\dot{\alpha}} .
\end{aligned}
$$

The EOM are thus obtained by setting the Euler-Lagrange derivatives in (3.6) and (3.7) to zero. In particular (3.6) yields Einstein's field equations with a stress-energy tensor containing the gravitino and its derivatives. Notice that $R_{\mu \nu}{ }^{a b}$ contains gravitino dependent terms via the gravitino dependence of $\omega_{\mu}^{a b}$. Hence, in order to cast Einstein's field equations in the familiar form, one not only has to divide by $e$ and convert the Lorentz index $a$ into a world index by means of the vierbein, but in addition one has to separate the gravitino dependent terms contained in $R_{\mu \nu}^{a b}$ from those terms which depend only on the vierbein (the latter give rise to the standard Einstein tensor on the "left hand side" of Einstein's field equations).

\subsection{Gauge symmetries}

The nontrivial gauge symmetries of the SUGRA action (3.3) may be grouped into three types: 
1. Invariance under spacetime diffeomorphisms with four real gauge parameters $\xi^{\mu}$ :

$$
\begin{aligned}
\delta_{\text {diffeo }} e_{\mu}^{a} & =\xi^{\nu} \partial_{\nu} e_{\mu}^{a}+\partial_{\mu} \xi^{\nu} e_{\nu}^{a}, \\
\delta_{\text {diffeo }} \psi_{\mu} & =\xi^{\nu} \partial_{\nu} \psi_{\mu}+\partial_{\mu} \xi^{\nu} \psi_{\nu}, \\
\delta_{\text {diffeo }} \bar{\psi}_{\mu} & =\xi^{\nu} \partial_{\nu} \bar{\psi}_{\mu}+\partial_{\mu} \xi^{\nu} \bar{\psi}_{\nu} .
\end{aligned}
$$

The invariance under these transformations can be deduced from the fact that the Lagrangian is by construction a scalar density with weight one under spacetime diffeomorphisms, as is familiar from standard GR (the induced transformation of the spin connection (3.5) is $\left.\delta_{\text {diffeo }} \omega_{\mu}^{a b}=\xi^{\nu} \partial_{\nu} \omega_{\mu}^{a b}+\partial_{\mu} \xi^{\nu} \omega_{\nu}^{a b}\right)$.

2. Invariance under local Lorentz transformations with six real gauge parameters $\xi^{a b}=-\xi^{b a}$ :

$$
\begin{aligned}
\delta_{\text {Lorentz }} e_{\mu}^{a} & =\xi_{b}{ }^{a} e_{\mu}^{b}, \\
\delta_{\text {Lorentz }} \psi_{\mu}^{\alpha} & =\frac{1}{2} \xi^{a b}\left(\psi_{\mu} \sigma_{a b}\right)^{\alpha}, \\
\delta_{\text {Lorentz }} \bar{\psi}_{\mu}^{\dot{\alpha}} & =-\frac{1}{2} \xi^{a b}\left(\bar{\sigma}_{a b} \bar{\psi}_{\mu}\right)^{\dot{\alpha}} .
\end{aligned}
$$

The Lagrangian is invariant under local Lorentz transformations because it is composed of Lorentz-covariant objects whose Lorentz-vector and spinor indices are "correctly contracted" (the induced transformation of the spin connection is $\delta_{\text {Lorentz }} \omega_{\mu}^{a b}=\nabla_{\mu} \xi^{a b}=\partial_{\mu} \xi^{a b}-\omega_{\mu c}^{a} \xi^{c b}-\omega_{\mu c}^{b} \xi^{a c}$, i.e., $\omega_{\mu}^{a b}$ transforms indeed as a gauge field for Lorentz transformations; $R_{\mu \nu}{ }^{a b}$ is Lorentz-covariant because it is the field strength of $\omega_{\mu}^{a b}$, and $\nabla_{\mu}$ is the Lorentz-covariant derivative).

3. Local SUSY with gauge parameters $\xi^{\alpha}$ that are complex Weyl spinors (and thus make up four real gauge parameters):

$$
\begin{aligned}
\delta_{\text {susy }} e_{\mu}^{a} & =2 \mathrm{i} \xi \sigma^{a} \bar{\psi}_{\mu}-2 \mathrm{i} \psi \psi_{\mu} \sigma^{a} \bar{\xi} \\
\delta_{\text {susy }} \psi_{\mu}^{\alpha} & =\nabla_{\mu} \xi^{\alpha}=\partial_{\mu} \xi^{\alpha}-\frac{1}{2} \omega_{\mu}^{a b}\left(\xi \sigma_{a b}\right)^{\alpha}, \\
\delta_{\text {susy }} \bar{\psi}_{\mu}^{\dot{\alpha}} & =\nabla_{\mu} \bar{\xi}^{\dot{\alpha}}=\partial_{\mu} \bar{\xi}^{\dot{\alpha}}+\frac{1}{2} \omega_{\mu}^{a b}\left(\bar{\sigma}_{a b} \bar{\xi}\right)^{\dot{\alpha}} .
\end{aligned}
$$

The invariance under these transformations is explicitly demonstrated in appendix B.1 using the "1.5 order formalism".

\subsection{Algebra of gauge transformations}

Let us first compute the commutator of two SUSY transformations on the vierbein. We shall use the notation $\delta_{\text {susy }}(\xi)$ meaning a SUSY transformation with parameters $\xi^{\alpha}$, and analogous notation for diffeomorphism and local Lorentz transformations.

$$
\begin{aligned}
{\left[\delta_{\text {susy }}\left(\xi_{1}\right), \delta_{\text {susy }}\left(\xi_{2}\right)\right] e_{\mu}^{a} } & =\delta_{\text {susy }}\left(\xi_{1}\right)\left(2 \mathrm{i} \xi_{2} \sigma^{a} \bar{\psi}_{\mu}-2 \mathrm{i} \psi_{\mu} \sigma^{a} \bar{\xi}_{2}\right)-(1 \leftrightarrow 2) \\
& =2 \mathrm{i} \xi_{2} \sigma^{a} \nabla_{\mu} \bar{\xi}_{1}-2 \mathrm{i} \nabla_{\mu} \xi_{1} \sigma^{a} \bar{\xi}_{2}-2 \mathrm{i} \xi_{1} \sigma^{a} \nabla_{\mu} \bar{\xi}_{2}+2 \mathrm{i} \nabla_{\mu} \xi_{2} \sigma^{a} \bar{\xi}_{1} \\
& =\nabla_{\mu}\left(2 \mathrm{i} \xi_{2} \sigma^{a} \bar{\xi}_{1}-2 \mathrm{i} \xi_{1} \sigma^{a} \bar{\xi}_{2}\right) .
\end{aligned}
$$


Notice that this expression does not contain derivatives of the gravitino and at most first order derivatives of the vierbein. Hence, in the second order formulation it cannot contain a trivial gauge transformation discussed in section 2.4 because the Euler-Lagrange derivatives (3.6) and (3.7) contain second order derivatives of the vierbein and first order derivatives of the gravitino, respectively. Therefore the commutator (3.17) should be a combination of the gauge transformations (3.8), (3.11) and (3.14) with composite parameters depending on the fields and the gauge parameters $\xi_{1}^{\alpha}, \xi_{2}^{\alpha}$ (and their derivatives). To verify that this is indeed the case, we examine a general gauge transformation of the vierbein (diffeomorphism + local Lorentz + SUSY transformation):

$$
\begin{aligned}
& \delta_{\text {gauge }} e_{\mu}^{a}=\xi^{\nu} \partial_{\nu} e_{\mu}^{a}+\partial_{\mu} \xi^{\nu} e_{\nu}^{a}+\xi_{b}{ }^{a} e_{\mu}^{b}+2 \mathrm{i} \xi \sigma^{a} \bar{\psi}_{\mu}-2 \mathrm{i} \psi_{\mu} \sigma^{a} \bar{\xi}
\end{aligned}
$$

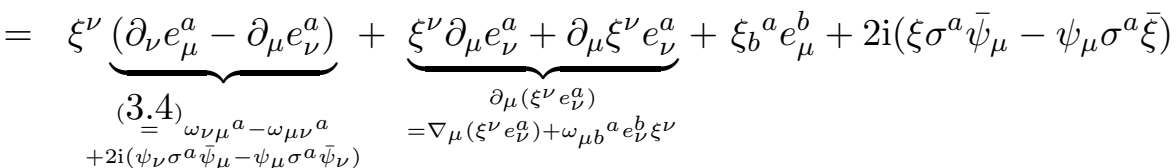

$$
\begin{aligned}
& =\nabla_{\mu}\left(\xi^{\nu} e_{\nu}^{a}\right)+\left(\xi_{b}^{a}+\xi^{\nu} \omega_{\nu b}^{a}\right) e_{\mu}^{b}+2 \mathrm{i}\left(\xi+\xi^{\nu} \psi_{\nu}\right) \sigma^{a} \bar{\psi}_{\mu}-2 \mathrm{i} \psi_{\mu} \sigma^{a}\left(\bar{\xi}+\xi^{\nu} \bar{\psi}_{\nu}\right) \text {. }
\end{aligned}
$$

Hence we have

$$
\delta_{\text {gauge }} e_{\mu}^{a}=\nabla_{\mu} \hat{\xi}^{a}+\hat{\xi}_{b}^{a} e_{\mu}^{b}+2 \mathrm{i} \hat{\xi} \sigma^{a} \bar{\psi}_{\mu}-2 \mathrm{i} \psi_{\mu} \sigma^{a} \overline{\hat{\xi}}
$$

where

$$
\hat{\xi}^{a}=\xi^{\nu} e_{\nu}^{a}, \quad \hat{\xi}^{a b}=\xi^{a b}+\xi^{\nu} \omega_{\nu}^{a b}, \quad \hat{\xi}^{\alpha}=\xi^{\alpha}+\xi^{\nu} \psi_{\nu}^{\alpha}, \quad \hat{\hat{\xi}}^{\dot{\alpha}}=\bar{\xi}^{\dot{\alpha}}+\xi^{\nu} \bar{\psi}_{\nu}^{\dot{\alpha}}
$$

Consider now gauge transformations with $\hat{\xi}^{a b}=0$ and $\hat{\xi}^{\alpha}=0$. These are combinations of diffeomorphism transformations of $e_{\mu}^{a}$ with parameters $\xi^{\nu}$, Lorentz transformations of $e_{\mu}^{a}$ with composite parameters $\xi^{a b}=-\xi^{\nu} \omega_{\nu}^{a b}$ (as this is equivalent to $\hat{\xi}^{a b}=0$ ), and SUSY transformations of $e_{\mu}^{a}$ with composite parameters $\xi^{\alpha}=-\xi^{\nu} \psi_{\nu}^{\alpha}\left(\Leftrightarrow \hat{\xi}^{\alpha}=0\right)$. Since the right hand side of (3.18) reduces for $\hat{\xi}^{a b}=\hat{\xi}^{\alpha}=0$ to $\nabla_{\mu} \hat{\xi}^{a}$, one has thus:

$$
\delta_{\text {diffeo }}\left(\xi^{\nu}\right) e_{\mu}^{a}+\delta_{\text {Lorentz }}\left(-\xi^{\nu} \omega_{\nu}^{a b}\right) e_{\mu}^{a}+\delta_{\text {susy }}\left(-\xi^{\nu} \psi_{\nu}^{\alpha}\right) e_{\mu}^{a}=\nabla_{\mu} \hat{\xi}^{a} .
$$

Using this in (3.17) we obtain that, on the vielbein, $\left[\delta_{\text {susy }}\left(\xi_{1}\right), \delta_{\text {susy }}\left(\xi_{2}\right)\right]$ is the sum of a diffeomorphism transformation with parameters $\xi_{1,2}^{\nu}=2 \mathrm{i}\left(\xi_{2} \sigma^{\nu} \bar{\xi}_{1}-\xi_{1} \sigma^{\nu} \bar{\xi}_{2}\right)$, a local Lorentz transformation with parameters $-\xi_{1,2}^{\nu} \omega_{\nu}{ }^{a b}$ and a SUSY transformation with parameters $-\xi_{1,2}^{\nu} \psi_{\nu}^{\alpha}$. On the gravitino this holds only on-shell as can be explicitly verified but the computation is cumbersome because one must compute $\delta_{\text {susy }} \omega_{\mu}{ }^{a b}$ with $\omega_{\mu}{ }^{a b}$ given by (3.5), and use the EOM of the gravitino. We shall not perform this computation here because its result can be obtained more elegantly from the supercovariant tensor calculus to be discussed later. One obtains thus

$$
\begin{aligned}
& {\left[\delta_{\text {susy }}\left(\xi_{1}\right), \delta_{\text {susy }}\left(\xi_{2}\right)\right]=} \delta_{\text {diffeo }}\left(\xi_{1,2}^{\nu}\right)+\delta_{\text {Lorentz }}\left(-\xi_{1,2}^{\nu} \omega_{\nu}^{a b}\right)+\delta_{\text {susy }}\left(-\xi_{1,2}^{\nu} \psi_{\nu}^{\alpha}\right)+\delta^{\text {triv }} \\
& \text { with } \xi_{1,2}^{\nu}=2 \mathrm{i}\left(\xi_{2} \sigma^{\nu} \bar{\xi}_{1}-\xi_{1} \sigma^{\nu} \bar{\xi}_{2}\right),
\end{aligned}
$$


where $\delta^{\text {triv }}$ is a trivial gauge transformation as in section 2.4 involving the Euler-Lagrange derivatives (3.7). The remaining part of the algebra is quite standard and can be easily derived:

$$
\begin{aligned}
& {\left[\delta_{\text {diffeo }}\left(\xi_{1}\right), \delta_{\text {diffeo }}\left(\xi_{2}\right)\right]=\delta_{\text {diffeo }}\left(\xi_{1,2}\right) \quad \text { with } \quad \xi_{1,2}^{\mu}=\xi_{2}^{\nu} \partial_{\nu} \xi_{1}^{\mu}-\xi_{1}^{\nu} \partial_{\nu} \xi_{2}^{\mu},} \\
& {\left[\delta_{\text {Lorentz }}\left(\xi_{1}\right), \delta_{\text {Lorentz }}\left(\xi_{2}\right)\right]=\delta_{\text {Lorentz }}\left(\xi_{1,2}\right) \quad \text { with } \quad \xi_{1,2}^{a b}=\xi_{1}^{a c} \xi_{2 c}{ }^{b}-\xi_{2}^{a c} \xi_{1 c}{ }^{b} \text {, }} \\
& {\left[\delta_{\text {diffeo }}\left(\xi_{1}\right), \delta_{\text {Lorentz }}\left(\xi_{2}\right)\right]=\delta_{\text {Lorentz }}\left(\xi_{1,2}\right) \quad \text { with } \quad \xi_{1,2}^{a b}=-\xi_{1}^{\mu} \partial_{\mu} \xi_{2}^{a b},} \\
& {\left[\delta_{\text {diffeo }}\left(\xi_{1}\right), \delta_{\text {susy }}\left(\xi_{2}\right)\right]=\delta_{\text {susy }}\left(\xi_{1,2}\right) \quad \text { with } \quad \xi_{1,2}^{\alpha}=-\xi_{1}^{\mu} \partial_{\mu} \xi_{2}^{\alpha},} \\
& {\left[\delta_{\text {Lorentz }}\left(\xi_{1}\right), \delta_{\text {susy }}\left(\xi_{2}\right)\right]=\delta_{\text {susy }}\left(\xi_{1,2}\right) \quad \text { with } \quad \xi_{1,2}^{\alpha}=-\frac{1}{2} \xi_{1}^{a b}\left(\xi_{2} \sigma_{a b}\right)^{\alpha} \text {. }}
\end{aligned}
$$

Owing to the trivial gauge transformations in (3.21) the algebra is open. This is one difference as compared to simpler gauge theories such as YM theory or standard GR. Another difference is that the composite parameters of the gauge transformations which occur on the right hand side of (3.21) are field dependent, whereas in YM theory or standard GR one has $\left[\delta_{\xi_{1}}, \delta_{\xi_{2}}\right]=\delta_{\xi_{1,2}}$ with $\xi_{1,2}$ depending only on $\xi_{1}, \xi_{2}$ and their derivatives, as in (3.22) - (3.26) .

Remark: Note that the $\hat{\xi}$ in (3.19) are related to the $\xi$ by gauge parameter redefinitions of the type discussed already in section 2.5, namely $\hat{\xi}^{N}=K_{M}^{N} \xi^{M}$ with field

dependent $K_{M}^{N}$. We are free to use the $\hat{\xi}$ as gauge parameters instead of the $\xi$. As explained in section 2.5, this is equivalent to changing the generating set of gauge transformations. This alternative form of the gauge transformations arises naturally within an approach to SUGRA based on a supercovariant tensor calculus to be discussed in the following sections. The gauge transformations of the vierbein in terms of these parameters are given by (3.18), the corresponding transformations of the gravitino read:

$$
\begin{gathered}
\delta_{\text {gauge }} \psi_{\mu}^{\alpha}=\xi^{\nu} \partial_{\nu} \psi_{\mu}^{\alpha}+\partial_{\mu} \xi^{\nu} \psi_{\nu}^{\alpha}+\frac{1}{2}\left(\hat{\xi}^{a b}-\xi^{\nu} \omega_{\nu}^{a b}\right)\left(\psi_{\mu} \sigma_{a b}\right)^{\alpha}+\nabla_{\mu}\left(\hat{\xi}^{\alpha}-\xi^{\nu} \psi_{\nu}^{\alpha}\right) \\
\Leftrightarrow \delta_{\text {gauge }} \psi_{\mu}^{\alpha}=\hat{\xi}^{a} E_{a}^{\nu}\left(\nabla_{\nu} \psi_{\mu}-\nabla_{\mu} \psi_{\nu}\right)^{\alpha}+\frac{1}{2} \hat{\xi}^{a b}\left(\psi_{\mu} \sigma_{a b}\right)^{\alpha}+\nabla_{\mu} \hat{\xi}^{\alpha}
\end{gathered}
$$

\section{Tensor calculus for standard gauge theories}

So far we discussed pure $D=4, N=1$ SUGRA with field content made up only of the vierbein and gravitino fields. In that basic formulation the gauge transformations form an open algebra in the terminology of section 2.5. There is an alternative formulation [20, 21], often called "off-shell formulation" because in that formulation the commutator algebra of the gauge transformations closes off-shell. This is made possible by the inclusion of additional fields which do not carry physical degrees of freedom and can be eliminated algebraically using their equations of motion (analogously to the spin connection $\omega_{\mu}^{a b}$ in the first order fomulation, see section 3.1). Therefore they are called auxiliary fields. Elimination of the auxiliary fields reproduces the "on-shell formulation" of pure $D=4, N=1$ SUGRA discussed in section 3 . An off-shell formulation does not only exist for pure $D=4, N=1$ SUGRA but also for its coupling to standard "matter multiplets" which is of great help for the construction of matter couplings to $D=4$, $N=1$ SUGRA. 
These off-shell formulations can be derived within a scheme that is not restricted to $D=4, N=1$ SUGRA but extends to a more general class of gauge theories. I refer to this class of gauge theories as standard gauge theories because it is characterized by properties familiar from YM theories or GR. The scheme itself may be called "tensor calculus for standard gauge theories" and is presented in this section 3 . In section 5 we shall specify how it can be used to derive the off-shell formulation of $D=4, N=1$ SUGRA.

\subsection{Basic input}

The tensor calculus centers round the notion of gauge covariance, in particular gauge covariant quantities and operations, such as tensor fields and covariant derivatives. Its structure resembles properties familiar from YM theories and GR. However we shall introduce it in a somewhat unfamiliar manner which starts off from formulae for the gauge transformations and the "partial derivatives" $\left(\partial_{\mu}\right)$ of tensor fields. The formula for the gauge transformations characterizes tensor fields through a certain transformation law and is thus analogous to the definition of tensor fields in GR through transformation properties under general coordinate transformation, for instance. The formula for the derivatives of tensor fields is an unusual but quite useful way to introduce gauge covariant derivatives.

We denote the gauge parameters by $\hat{\xi}^{M}$. The hat on $\xi$ indicates that these parameters might correspond to an unusal formulation of the gauge transformations. For instance, in pure SUGRA this formulation corresponds to the parameters in equation (3.19) rather than to those used in section 3.3 . At the end of section 4.2 we shall cast the gauge transformations in more standard form with "unhatted" parameters. Tensor fields are now characterized as follows: a tensor field $T$ is a local function of the fields whose gauge transformations do not contain derivatives of gauge parameters $\hat{\xi}^{M}$ and thus take the form $\delta_{\hat{\xi}} T=\hat{\xi}^{M} X_{M}$, for some local functions $X_{M}$. Moreover we require that these functions are themselves tensor fields and that they can be written in terms of operators $\Delta_{M}$ (graded derivations, see below) according to $\Delta_{M} T=X_{M}$. Basically, the latter just means that we can define $\Delta_{M}$ on $T$ through $\Delta_{M} T:=X_{M}$. Hence, tensor fields transform in this setting according to

$$
\delta_{\hat{\xi}} T=\hat{\xi}^{M} \Delta_{M} T \text {. }
$$

This is the formula for the gauge transformations of tensor fields announced above. The formula for the derivatives of tensor fields takes a similar form. In terms of the exterior derivative on the jet space, $d=d x^{\mu} \partial_{\mu}$, it reads

$$
d T=A^{M} \Delta_{M} T
$$

This expresses the exterior derivative of a tensor field as a linear combination of the operations $\Delta_{M}$ with coefficients that are 1-forms $A^{M}$ (because $d$ has form-degree 1 ). In

\footnotetext{
${ }^{3}$ Actually the scheme can be extended to rather general gauge theories and thus to a general tensor calculus [22, 23] but the explanation of this extension is beyond the scope of this work.
} 
general these 1-forms will not be tensor fields because $d T=d x^{\mu} \partial_{\mu} T$ is a combination of the derivatives of $T$ which are usually not tensor fields (cf. GR or YM theories). Rather we shall see that the $A^{M}$ should be interpreted as "connections" built of gauge fields $A_{\mu}^{M}$ according to

$$
A^{M}=d x^{\mu} A_{\mu}^{M}
$$

(4.2) will now be used to introduce gauge covariant derivatives. To that end we assume that a subset of the gauge fields $A_{\mu}^{M}$ forms a field dependent invertible matrix (in the SUGRA case this will be the vierbein). We denote that subset by $\left\{V_{\mu}^{a}\right\}$, and the remaining gauge fields by $A_{\mu}^{\hat{M}}$ where we have split the index set $\{M\}$ into subsets $\{a\}$ and $\{\hat{M}\}$ :

$$
\{M\}=\{a, \hat{M}\}, \quad\left\{A_{\mu}^{M}\right\}=\left\{V_{\mu}^{a}, A_{\mu}^{\hat{M}}\right\}, \quad a \in\{0, \ldots, D-1\} .
$$

Equation (4.2) can now be interpreted as a definition of the operators $\Delta_{a}$ :

$$
\Delta_{a} T=\left(V^{-1}\right)_{a}^{\mu}\left(\partial_{\mu}-A_{\mu}^{\hat{M}} \Delta_{\hat{M}}\right) T .
$$

Notice that $\Delta_{a}$ has a form analogous to covariant derivatives in YM theory or GR. Therefore we interpret it as a gauge covariant derivative. It is indeed gauge covariant if $\Delta_{M} T$ is a tensor field for any $M$ and every tensor field $T$, as we have assumed. Let us elaborate in some more detail on this assumption. It demands that the $\Delta$ 's are graded derivations in the space of tensor fields, i.e., they map tensor fields to tensor fields and satisfy the Leibniz rule

$$
\Delta_{M}\left(T_{1} T_{2}\right)=\left(\Delta_{M} T_{1}\right) T_{2}+(-)^{|M|\left|T_{1}\right|} T_{1}\left(\Delta_{M} T_{2}\right),
$$

where $|M|$ denotes the Grassmann parity of the gauge parameter $\hat{\xi}^{M}$. (4.6) must hold because the gauge transformations are to be Grassmann even derivations, cf. (2.13), and shows that $\Delta_{M}$ has the same Grassmann parity as the corresponding gauge parameter; moreover $\Delta_{a}$ should have even Grassmann parity (the same as $\partial_{\mu}$ ),

$$
\left|\Delta_{M}\right|=\left|\hat{\xi}^{M}\right|=|M|, \quad\left|\Delta_{a}\right|=\left|\hat{\xi}^{a}\right|=|a|=0 .
$$

Owing to (4.2) (and because $d$ is Grassmann odd, as it contains the differentials $d x^{\mu}$ ), this also fixes the Grassmann parities of the gauge fields:

$$
\left|A^{M}\right|=|M|+1 \quad(\bmod 2), \quad\left|A_{\mu}^{M}\right|=|M| .
$$

Remark: (4.1) and (4.2) establish a formal similarity of the gauge transformations and the derivatives of tensor fields which might be surprising at first glance. However, at a second glance it makes quite some sense: from a purely algebraic point of view (in particular in the jet space approach) the gauge transformations and the derivatives are actually quite similar and differ basically only in their commutation relations (the derivatives are required to commute among themselves and with the gauge transformations, whereas the latter in general do not necessarily commute among themselves, see equations (4.9) through (4.11) below). Furthermore, it may be worthwhile to compare with the fiber bundle formulation of YM theories: there the gauge transformations and the partial derivatives are also similar operations in the sense that the former correspond to displacements in the fiber, the latter to displacements in the base manifold. 


\subsection{Consistency requirements}

We proceed by working out the consistency conditions which must be satisfied in order that (4.1) and (4.2) can provide an off-shell formulation of a gauge theory. These consistency conditions arise from the algebra of gauge transformations and partial derivatives which is to read

$$
\begin{aligned}
& {\left[\delta_{\hat{\xi}_{1}}, \delta_{\hat{\xi}_{2}}\right]=\delta_{f}, \quad f^{M}=f^{M}\left(x,\left[\hat{\xi}_{1}, \hat{\xi}_{2}, \phi\right]\right),} \\
& {\left[d, \delta_{\hat{\xi}}\right]=0} \\
& d^{2}=0 .
\end{aligned}
$$

In (4.9), $\delta_{f}$ is to be a gauge transformation of the same form as $\delta_{\hat{\xi}_{1}}$ and $\delta_{\hat{\xi}_{2}}$, but with "composite parameters" $f^{M}$, in order that the commutator algebra of the gauge transformations closes off-shell. (4.10) is equivalent to $\left[\partial_{\mu}, \delta_{\hat{\xi}}\right]=0$ and thus expresses (2.11). (4.11) is equivalent to $\left[\partial_{\mu}, \partial_{\nu}\right]=0$ and is included because (4.2) is to be consistent with these basic commutation relations of the derivatives.

We start with the commutator of two gauge transformations on tensor fields. Using (4.1) and that the $\Delta_{M} T$ are tensor fields, we obtain

$$
\begin{aligned}
{\left[\delta_{\hat{\xi}_{1}}, \delta_{\hat{\xi}_{2}}\right] T } & =\delta_{\hat{\xi}_{1}}\left(\hat{\xi}_{2}^{N} \Delta_{N} T\right)-(1 \leftrightarrow 2)=\hat{\xi}_{2}^{N} \hat{\xi}_{1}^{M} \Delta_{M} \Delta_{N} T-\hat{\xi}_{1}^{N} \hat{\xi}_{2}^{M} \Delta_{M} \Delta_{N} T \\
& =\hat{\xi}_{2}^{N} \hat{\xi}_{1}^{M}\left[\Delta_{M}, \Delta_{N}\right] T
\end{aligned}
$$

where $[$,$] is the graded commutator$

$$
[X, Y]=X Y-(-)^{|X||Y|} Y X .
$$

On a tensor field, the right hand side of (4.9) must again be a gauge transformation of the form (4.1) when we impose off-shell closure of the gauge algebra, i.e., it must be a combination of the $\Delta_{M} T$ with certain coefficient functions $f^{M}$. Since the gauge transformations of a tensor field do not involve derivatives of the gauge parameters, these coefficient functions do not involve derivatives of the $\hat{\xi}$ 's, cf. (4.12). Hence we require

$$
\left[\delta_{\hat{\xi}_{1}}, \delta_{\hat{\xi}_{2}}\right] T=\hat{\xi}_{1}^{M} \hat{\xi}_{2}^{N} \mathcal{F}_{N M}^{P} \Delta_{P} T,
$$

for some tensor fields $\mathcal{F}_{M N}{ }^{P}$ [that these must be tensor fields is also seen by comparing with (4.12), since $\Delta_{M} T$ is to be a tensor field whenever $T$ is]. As $\left[\delta_{\hat{\xi}_{1}}, \delta_{\hat{\xi}_{2}}\right]$ is skewsymmetric under exchange of $\hat{\xi}_{1}$ and $\hat{\xi}_{2}$, these tensor fields are subject to the symmetry property

$$
\mathcal{F}_{M N}{ }^{P}=-(-)^{|M||N|} \mathcal{F}_{N M}{ }^{P} .
$$

Since (4.12) and (4.14) must coincide for all gauge parameters and all tensor fields, we require that the $\Delta$ 's satisfy the graded commutator algebrat

$$
\left[\Delta_{M}, \Delta_{N}\right]=-\mathcal{F}_{M N}{ }^{P} \Delta_{P}
$$

\footnotetext{
${ }^{4}$ Note that (4.16) is a sufficient condition for the compatibility of (4.12) and (4.14). In special cases it might not be a necessary condition. Analogously, equations (4.21) and (4.23) are only sufficient for consistency, but in general not necessary.
} 
[The minus sign is due to $\hat{\xi}_{1}^{M} \hat{\xi}_{2}^{N} \mathcal{F}_{N M}{ }^{P}=-\hat{\xi}_{2}^{N} \hat{\xi}_{1}^{M} \mathcal{F}_{M N}{ }^{P}$.] This algebra implies consistency conditions for the tensor fields $\mathcal{F}_{M N}{ }^{P}$ and their $\Delta$-transformations. These follow from the following identity for graded commutators:

$$
\sum_{M N P}\left[\Delta_{M},\left[\Delta_{N}, \Delta_{P}\right]\right]=0
$$

where the graded cyclic sum was used defined by

$$
\sum_{M N P} X_{M N P}=(-)^{|M||P|} X_{M N P}+(-)^{|N||M|} X_{N P M}+(-)^{|P||N|} X_{P M N} .
$$

(4.16) and (4.17) yield

$$
\sum_{M N P}\left(\Delta_{M} \mathcal{F}_{N P}^{Q}+\mathcal{F}_{M N}^{R} \mathcal{F}_{R P}^{Q}\right)=0
$$

As we shall see, these equations are the crucial consistency requirements.

Next we consider the commutators of the exterior derivative and gauge transformations on tensor fields. Using (4.1), (4.2), (4.16) and that the $\Delta_{M} T$ are tensor fields, we obtain

$$
\begin{aligned}
{\left[d, \delta_{\hat{\xi}}\right] T } & =d\left(\hat{\xi}^{M} \Delta_{M} T\right)-\delta_{\hat{\xi}}\left(A^{M} \Delta_{M} T\right) \\
& =\left(d \hat{\xi}^{M}\right) \Delta_{M} T+(-)^{|M|} \hat{\xi}^{M} d\left(\Delta_{M} T\right)-\left(\delta_{\hat{\xi}} A^{M}\right) \Delta_{M} T-A^{M} \delta_{\hat{\xi}}\left(\Delta_{M} T\right) \\
& =\left(d \hat{\xi}^{M}\right) \Delta_{M} T+(-)^{|M|} \hat{\xi}^{M} A^{N} \Delta_{N} \Delta_{M} T-\left(\delta_{\hat{\xi}} A^{M}\right) \Delta_{M} T-A^{M} \hat{\xi}^{N} \Delta_{N} \Delta_{M} T \\
& =\left(d \hat{\xi}^{M}-\delta_{\hat{\xi}} A^{M}\right) \Delta_{M} T-A^{M} \hat{\xi}^{N}\left[\Delta_{N}, \Delta_{M}\right] T \\
& =\left(d \hat{\xi}^{M}-\delta_{\hat{\xi}} A^{M}+A^{P} \hat{\xi}^{N} \mathcal{F}_{N P}{ }^{M} \Delta_{M} T .\right.
\end{aligned}
$$

According to (4.10), these commutators must vanish for all $T$. Therefore we require that the sum of the terms in parantheses in the last line of (4.20) vanishes for each $M$. This fixes the gauge transformations of the connections:

$$
\delta_{\hat{\xi}} A^{M}=d \hat{\xi}^{M}+A^{P} \hat{\xi}^{N} \mathcal{F}_{N P}{ }^{M}
$$

i.e., for the gauge fields:

$$
\delta_{\hat{\xi}} A_{\mu}^{M}=\partial_{\mu} \hat{\xi}^{M}+A_{\mu}^{P} \hat{\xi}^{N} \mathcal{F}_{N P}{ }^{M}
$$

Last but not least we compute $d^{2}$ on tensor fields using (4.2). We obtain

$$
\begin{aligned}
d^{2} T & =d\left(A^{M} \Delta_{M} T\right) \\
& =\left(d A^{M}\right) \Delta_{M} T+(-)^{|M|+1} A^{M} d\left(\Delta_{M} T\right) \\
& =\left(d A^{M}\right) \Delta_{M} T+(-)^{|M|+1} A^{M} A^{N} \Delta_{N} \Delta_{M} T \\
& =\left(d A^{M}\right) \Delta_{M} T+\frac{1}{2}(-)^{|M|+1}\left(A^{M} A^{N}+(-)^{(|M|+1)(|N|+1)} A^{N} A^{M}\right) \Delta_{N} \Delta_{M} T
\end{aligned}
$$




$$
\begin{aligned}
& =\left(d A^{M}\right) \Delta_{M} T+\frac{1}{2}(-)^{|M|+1} A^{M} A^{N} \Delta_{N} \Delta_{M} T+\frac{1}{2}(-)^{(|M|+1)|N|} A^{N} A^{M} \Delta_{N} \Delta_{M} T \\
& =\left(d A^{M}\right) \Delta_{M} T+\frac{1}{2}(-)^{|M|+1} A^{M} A^{N} \Delta_{N} \Delta_{M} T+\frac{1}{2}(-)^{(|N|+1)|M|} A^{M} A^{N} \Delta_{M} \Delta_{N} T \\
& =\left(d A^{M}\right) \Delta_{M} T+\frac{1}{2}(-)^{|M|+1} A^{M} A^{N}\left(\Delta_{N} \Delta_{M}+(-)^{(|N|+1)|M|+|M|+1} \Delta_{M} \Delta_{N}\right) T \\
& =\left(d A^{M}\right) \Delta_{M} T+\frac{1}{2}(-)^{|M|+1} A^{M} A^{N}\left(\Delta_{N} \Delta_{M}-(-)^{|N||M|} \Delta_{M} \Delta_{N}\right) T \\
& =\left(d A^{M}\right) \Delta_{M} T+\frac{1}{2}(-)^{|M|+1} A^{M} A^{N}\left[\Delta_{N}, \Delta_{M}\right] T \\
& =\left(d A^{M}-\frac{1}{2}(-)^{|P|+1} A^{P} A^{N} \mathcal{F}_{N P}{ }^{M}\right) \Delta_{M} T
\end{aligned}
$$

where we used (4.2), (4.8), (4.16) and, again, that the $\Delta_{M} T$ are tensor fields [note that (4.8) implies $\left.A^{M} A^{N}=(-)^{(|M|+1)(|N|+1)} A^{N} A^{M}\right]$. As $d^{2} T$ must vanish for all $T$ we require

$$
d A^{M}+\frac{1}{2}(-)^{|P|} A^{P} A^{N} \mathcal{F}_{N P}{ }^{M}=0
$$

This equation looks at first glance like a differential equation for $A^{M} 5$ However, actually it determines the curvatures of the covariant derivatives [this is similar - and related to the fact that (4.2) is no differential equation for tensor fields but the definition of the covariant derivatives]. To see this we spell it out in components. Using

$$
d A^{M}=d x^{\mu} \partial_{\mu} A^{M}=d x^{\mu} d x^{\nu} \partial_{\mu} A_{\nu}^{M}=\frac{1}{2} d x^{\mu} d x^{\nu}\left(\partial_{\mu} A_{\nu}^{M}-\partial_{\nu} A_{\mu}^{M}\right)
$$

and

$$
A^{P} A^{N}=d x^{\mu} A_{\mu}^{P} d x^{\nu} A_{\nu}^{N}=(-)^{|P|} d x^{\mu} d x^{\nu} A_{\mu}^{P} A_{\nu}^{N}
$$

we obtain from (4.23)

$$
\partial_{\mu} A_{\nu}^{M}-\partial_{\nu} A_{\mu}^{M}+A_{\mu}^{P} A_{\nu}^{N} \mathcal{F}_{N P}^{M}=0
$$

[One has $A_{\mu}^{P} A_{\nu}^{N} \mathcal{F}_{N P}{ }^{M}=-A_{\nu}^{P} A_{\mu}^{N} \mathcal{F}_{N P}{ }^{M}$ owing to (4.8) and (4.15).] Now, $A_{\mu}^{P} A_{\nu}^{N} \mathcal{F}_{N P}{ }^{M}$ contains $V_{\mu}^{a} V_{\nu}^{b} \mathcal{F}_{b a}{ }^{M}$, cf. (4.4). We can thus write (4.24) as

$$
V_{\mu}^{a} V_{\nu}^{b} \mathcal{F}_{a b}{ }^{M}=\partial_{\mu} A_{\nu}^{M}-\partial_{\nu} A_{\mu}^{M}+A_{\mu}^{\hat{P}} A_{\nu}^{\hat{N}} \mathcal{F}_{\hat{N} \hat{P}}{ }^{M}+V_{\mu}^{a} A_{\nu}^{\hat{N}} \mathcal{F}_{\hat{N} a}{ }^{M}-V_{\nu}^{a} A_{\mu}^{\hat{N}} \mathcal{F}_{\hat{N} a}{ }^{M}
$$

where we used $\mathcal{F}_{a N}{ }^{M}=-\mathcal{F}_{N a}{ }^{M}$ which follows from (4.15) owing to $|a|=0$, see (4.7). As $V$ is assumed to be invertible, (4.25) can be solved for $\mathcal{F}_{c d}{ }^{M}$ by contracting it with $\left(V^{-1}\right)_{c}^{\mu}$ and $\left(V^{-1}\right)_{d}^{\nu}$. Hence (4.23) can be viewed as an equation for the $\mathcal{F}_{a b}{ }^{M}$ which can indeed be interpreted as curvatures or torsions for the covariant derivatives, as (4.16) reads for $M=a$ and $N=b$ :

$$
\left[\Delta_{a}, \Delta_{b}\right] T=-\mathcal{F}_{a b}{ }^{M} \Delta_{M} T \text {. }
$$

This ends the discussion of (4.9) through (4.11) on tensor fields. What about the gauge fields? It turns out that (4.9) through (4.11) do automatically hold also on the

\footnotetext{
${ }^{5}$ Notice also that it looks formally like a Maurer-Cartan equation, or a "zero-curvature condition". Actually it is indeed a zero-curvature condition, but just for the derivatives as it expresses $\left[\partial_{\mu}, \partial_{\nu}\right] T=0$.
} 
gauge fields as a consequence of (4.19), with the same $f^{M}$ as in (4.14) (note that the latter is required because the commutator algebra of the gauge transformations must of course coincide on tensor field and gauge fields in an off-shell formulation). Indeed one obtains, using the formulae derived so far:

$$
\begin{aligned}
{\left[\delta_{\hat{\xi}_{1}}, \delta_{\hat{\xi}_{2}}\right] A^{M}=} & \delta_{\hat{\xi}_{1}}\left(d \hat{\xi}_{2}^{M}+A^{P} \hat{\xi}_{2}^{N} \mathcal{F}_{N P}{ }^{M}\right)-(1 \leftrightarrow 2) \\
= & \left(\delta_{\hat{\xi}_{1}} A^{P}\right) \hat{\xi}_{2}^{N} \mathcal{F}_{N P}{ }^{M}+A^{P} \hat{\xi}_{2}^{N}\left(\delta_{\hat{\xi}_{1}} \mathcal{F}_{N P}{ }^{M}\right)-(1 \leftrightarrow 2) \\
= & \left(d \hat{\xi}_{1}^{P}+A^{Q} \hat{\xi}_{1}^{R} \mathcal{F}_{R Q}{ }^{P}\right) \hat{\xi}_{2}^{N} \mathcal{F}_{N P}{ }^{M}+A^{P} \hat{\xi}_{2}^{N} \hat{\xi}_{1}^{Q} \Delta_{Q} \mathcal{F}_{N P}{ }^{M}-(1 \leftrightarrow 2) \\
= & d\left(\hat{\xi}_{1}^{P} \hat{\xi}_{2}^{N} \mathcal{F}_{N P} P^{M}\right)+A^{P} \hat{\xi}_{1}^{Q} \hat{\xi}_{2}^{R} \mathcal{F}_{R Q}{ }^{P} \mathcal{F}_{N P}{ }^{M} \\
& +(-)^{|Q||P|} A^{P} \hat{\xi}_{2}^{N} \hat{\xi}_{1}^{Q} \sum_{M N P}\left(\Delta_{Q} \mathcal{F}_{N P}{ }^{M}+\mathcal{F}_{Q N}{ }^{R} \mathcal{F}_{R P}{ }^{M}\right), \\
{\left[d, \delta_{\hat{\xi}}\right] A^{M}=} & d\left(d \hat{\xi}^{M}+A^{P} \hat{\xi}^{N} \mathcal{F}_{N P}{ }^{M}\right)-\delta_{\hat{\xi}}\left(-\frac{1}{2}(-)^{|P|} A^{P} A^{N} \mathcal{F}_{N P}{ }^{M}\right)=\ldots \\
= & \frac{1}{2}(-)^{|P|(1+|Q|)} A^{P} A^{N} \hat{\xi}^{Q} \sum_{M N P}\left(\Delta_{Q} \mathcal{F}_{N P}{ }^{M}+\mathcal{F}_{Q N} \mathcal{F}_{R P}{ }^{M}\right), \\
d^{2} A^{M}= & d\left(-\frac{1}{2}(-)^{|P|} A^{P} A^{N} \mathcal{F}_{N P}{ }^{M}\right)=\ldots \\
= & -\frac{1}{6}(-)^{|N|+|Q||P|} A^{P} A^{N} A^{Q} \sum_{M N P}\left(\Delta_{Q} \mathcal{F}_{N P}{ }^{M}+\mathcal{F}_{Q N} \mathcal{F}_{R P}{ }^{M}\right) .(4.28
\end{aligned}
$$

Hence (4.9) through (4.11) are indeed satisfied on $A^{M}$ when (4.19) holds. This emphasizes the central importance of (4.19). Furthermore, we can now specify (4.9):

$$
\left[\delta_{\hat{\xi}_{1}}, \delta_{\hat{\xi}_{2}}\right]=\delta_{f}, \quad f^{P}=\hat{\xi}_{1}^{M} \hat{\xi}_{2}^{N} \mathcal{F}_{N M}^{P} \text {. }
$$

Let us finally rewrite the gauge transformations in terms of parameters $\xi^{\mu}, \xi^{\hat{M}}$ related to the $\hat{\xi}^{M}$ analogously to (3.19):

$$
\hat{\xi}^{a}=\xi^{\mu} V_{\mu}^{a}, \quad \hat{\xi}^{\hat{M}}=\xi^{\hat{M}}+\xi^{\mu} A_{\mu}^{\hat{M}} .
$$

For the gauge transformations of tensor fields we have

$$
\delta_{\hat{\xi}} T=\hat{\xi}^{M} \Delta_{M} T=\xi^{\mu} V_{\mu}^{a} \Delta_{a} T+\hat{\xi}^{\hat{M}} \Delta_{\hat{M}} T=\xi^{\mu}\left(\partial_{\mu}-A_{\mu}^{\hat{M}} \Delta_{\hat{M}}\right) T+\hat{\xi}^{\hat{M}} \Delta_{\hat{M}} T
$$

where we used $V_{\mu}^{a} \Delta_{a} T=\left(\partial_{\mu}-A_{\mu}^{\hat{M}} \Delta_{\hat{M}}\right) T$ which is nothing but a rewriting of (4.2). Hence the gauge transformations of tensor fields read in terms of the $\xi$ 's:

$$
\delta_{\xi} T=\xi^{\mu} \partial_{\mu} T+\xi^{\hat{M}} \Delta_{\hat{M}} T
$$

For the gauge transformations of the gauge fields $A_{\mu}^{\hat{M}}$ we obtain from (4.22):

$$
\begin{aligned}
\delta_{\hat{\xi}} A_{\mu}^{\hat{M}}= & \partial_{\mu} \hat{\xi}^{\hat{M}}+A_{\mu}^{P \hat{\xi}^{N}} \mathcal{F}_{N P} \hat{M} \\
= & \partial_{\mu}\left(\xi^{\hat{M}}+\xi^{\nu} A_{\nu}^{\hat{M}}\right)+A_{\mu}^{P}\left(\xi^{\hat{N}}+\xi^{\nu} A_{\nu}^{\hat{N}}\right) \mathcal{F}_{\hat{N} P}{ }^{\hat{M}}+A_{\mu}^{P} \xi^{\nu} V_{\nu}^{a} \mathcal{F}_{a P} \hat{M} \\
= & \partial_{\mu} \xi^{\hat{M}}+\partial_{\mu} \xi^{\nu} A_{\nu}^{\hat{M}}+\xi^{\nu}\left(\partial_{\mu} A_{\nu}^{\hat{M}}-\partial_{\nu} A_{\mu}^{\hat{M}}\right)+\xi^{\nu} \partial_{\nu} A_{\mu}^{\hat{M}} \\
& +A_{\mu}^{P}\left(\xi^{\hat{N}}+\xi^{\nu} A_{\nu}^{\hat{N}}\right) \mathcal{F}_{\hat{N} P} \hat{M}+A_{\mu}^{P} \xi^{\nu} V_{\nu}^{a} \mathcal{F}_{a P} \hat{M}
\end{aligned}
$$


Using now equation (4.24), i.e., $\partial_{\mu} A_{\nu}^{\hat{M}}-\partial_{\nu} A_{\mu}^{\hat{M}}=-A_{\mu}^{P} A_{\nu}^{N} \mathcal{F}_{N P} \hat{M}$, we obtain

$$
\delta_{\xi} A_{\mu}^{\hat{M}}=\xi^{\nu} \partial_{\nu} A_{\mu}^{\hat{M}}+\partial_{\mu} \xi^{\nu} A_{\nu}^{\hat{M}}+\partial_{\mu} \xi^{\hat{M}}+A_{\mu}^{P} \xi^{\hat{N}} \mathcal{F}_{\hat{N} P} \hat{M}
$$

An analogous computation for $V_{\mu}^{a}$ yields

$$
\delta_{\xi} V_{\mu}^{a}=\xi^{\nu} \partial_{\nu} V_{\mu}^{a}+\partial_{\mu} \xi^{\nu} V_{\nu}^{a}+A_{\mu}^{P} \xi^{\hat{N}} \mathcal{F}_{\hat{N} P}{ }^{a} .
$$

Notice that the right hand sides of equations (4.31), (4.32) and (4.33) involve $\xi^{\mu}$ only via the "Lie derivative terms" $\xi^{\mu} \partial_{\mu} T$ and $\xi^{\nu} \partial_{\nu} A_{\mu}^{M}+\partial_{\mu} \xi^{\nu} A_{\nu}^{M}$, respectively.

Remark: Formally the formulae above look quite familiar. For instance, (4.21) looks formally like the gauge transformations of a gauge field in YM theory if the $\mathcal{F}_{N P}{ }^{M}$ were the structure constants of a Lie algebra. However, in general (and in particular in SUGRA) the $\mathcal{F}_{N P}{ }^{M}$ are not constant but rather they are tensor fields, and therefore the algebra (4.16) is not a (graded) Lie algebra but a more general structure. In fact, Lie algebras are just the simplest examples of this structure, because in these examples the $\mathcal{F}_{N P}{ }^{M}$ are constants and (4.19) turns into the Jacobi identity for the structure constants of a Lie algebra. Hence (4.19) generalizes the Jacobi identity for Lie algebras to the more general algebras (4.16).

\section{Off-shell formulations of $\mathrm{D}=4, \mathrm{~N}=1$ SUGRA with matter}

\subsection{Supercovariant tensor calculus}

We shall now outline how an off-shell formulation of $\mathrm{D}=4, \mathrm{~N}=1$ SUGRA and its coupling to matter is obtained within the scheme described in section 4. The gauge symmetries to be implemented are in this case the spacetime diffeomorphisms, local Lorentz symmetry, SUSY and YM gauge symmetry. The corresponding "hatted" gauge parameters $\hat{\xi}$ are

$$
\left\{\hat{\xi}^{M}\right\}=\left\{\hat{\xi}^{a}, \hat{\xi}^{\alpha}, \overline{\hat{\xi}}^{\dot{\alpha}}, \hat{\xi}^{a b}, \hat{\xi}^{i}\right\}
$$

where the $\hat{\xi}^{i}$ are the hatted Yang-Mills gauge parameters, i.e., the index $i$ refers to some basis of the Lie algebra of a YM gauge group (for pure SUGRA, $\left\{\hat{\xi}^{i}\right\}$ is simply the empty set). The other gauge parameters and indices have already been introduced in section 3. The gauge fields $A_{\mu}^{M}$ are the vierbein $e_{\mu}^{a}$, the gravitino $\psi_{\mu}^{\alpha}$ and its complex conjugate $\bar{\psi}_{\mu}^{\dot{\alpha}}$, the spin connection $\omega_{\mu}^{a b}$ and Yang-Mills gauge fields $A_{\mu}^{i}$,

$$
\left\{A_{\mu}^{M}\right\}=\left\{e_{\mu}^{a}, \psi_{\mu}^{\alpha}, \bar{\psi}_{\mu}^{\dot{\alpha}}, \omega_{\mu}^{a b}, A_{\mu}^{i}\right\} .
$$

The vierbein is in this case identified with the gauge fields $V_{\mu}^{a}$ in (4.4),

$$
V_{\mu}^{a} \equiv e_{\mu}^{a}
$$

The $\Delta$-operations are denoted by

$$
\left\{\Delta_{M}\right\}=\left\{\mathcal{D}_{a}, \mathcal{D}_{\alpha}, \overline{\mathcal{D}}_{\dot{\alpha}}, l_{a b}, \delta_{i}\right\}
$$


Concerning summations over the indices $M$, we employ the following convention:

$$
X^{M} Y_{M} \equiv X^{a} Y_{a}+X^{\underline{\alpha}} Y_{\underline{\alpha}}+\frac{1}{2} X^{a b} Y_{a b}+X^{i} Y_{i}, \quad X^{\underline{\alpha}} Y_{\underline{\alpha}}=X^{\alpha} Y_{\alpha}+X_{\dot{\alpha}} Y^{\dot{\alpha}} .
$$

For instance, (4.2) reads thus explicitly in this case:

$$
\partial_{\mu} T=\left(e_{\mu}^{a} \mathcal{D}_{a}+\psi_{\mu}^{\alpha} \mathcal{D}_{\alpha}+\bar{\psi}_{\mu \dot{\alpha}} \overline{\mathcal{D}}^{\dot{\alpha}}+\frac{1}{2} \omega_{\mu}^{a b} l_{a b}+A_{\mu}^{i} \delta_{i}\right) T
$$

The covariant derivatives (4.5) are thus given by

$$
\mathcal{D}_{a} T=E_{a}^{\mu}\left(\partial_{\mu}-\psi_{\mu}^{\alpha} \mathcal{D}_{\alpha}-\bar{\psi}_{\mu \dot{\alpha}} \overline{\mathcal{D}}^{\dot{\alpha}}-\frac{1}{2} \omega_{\mu}^{a b} l_{a b}-A_{\mu}^{i} \delta_{i}\right) T .
$$

Notice that these covariant derivatives involve not only the spin connection and YangMills gauge fields, but in addition also the gravitino. They are thus covariant also with respect to local SUSY transformations. To distinguish them from the more familiar covariant derivatives in standard GR, we shall refer to them as supercovariant derivatives and to the corresponding tensor fields as supercovariant tensor fields. Notice also that $\mathcal{D}_{a}$ does not contain a connection $\Gamma_{\mu \nu}{ }^{\rho}$ for world indices. The reason is that all supercovariant tensor fields must be scalar fields with regard to spacetime diffeomorphisms because otherwise their gauge transformations would contain derivatives of the diffeomorphism parameters, in contradiction to the definition of tensor fields according to (4.1). Hence, according to this definition, supercovariant tensor fields do not carry world indices, and therefore a term with $\Gamma_{\mu \nu}{ }^{\rho}$ is not needed in $\mathcal{D}_{a}$. For the same reason the supercovariant derivatives themselves must be scalar operators with regard to diffeomorphisms which explains why the carry a Lorentz index instead of a world index.

\subsection{Bianchi identities}

$D=4, N=1$ SUGRA arises now by a suitable specification of the tensor fields $\mathcal{F}_{M N}{ }^{P}$ occurring in (4.16). This has to be done such that the consistency conditions (4.19) are satisfied. To describe this specification, we introduce the index sets $\{A\}=\{a, \alpha, \dot{\alpha}\}$ and $\{I\}=\{[a b], i\}$ so that (5.4) becomes

$$
\left\{\Delta_{M}\right\}=\left\{\mathcal{D}_{A}, \delta_{I}\right\}, \quad\left\{\mathcal{D}_{A}\right\}=\left\{\mathcal{D}_{a}, \mathcal{D}_{\alpha}, \overline{\mathcal{D}}_{\dot{\alpha}}\right\}, \quad\left\{\delta_{I}\right\}=\left\{l_{a b}, \delta_{i}\right\}
$$

The graded commutator algebra (4.16) for an off-shell formulation of $D=4, N=1$ SUGRA reads

$$
\begin{aligned}
{\left[\mathcal{D}_{A}, \mathcal{D}_{B}\right] } & =-T_{A B}{ }^{C} \mathcal{D}_{C}-F_{A B}{ }^{I} \delta_{I} \\
{\left[\delta_{I}, \mathcal{D}_{A}\right] } & =-g_{I A}{ }^{B} \mathcal{D}_{B} \\
{\left[\delta_{I}, \delta_{J}\right] } & =f_{I J}{ }^{K} \delta_{K} .
\end{aligned}
$$

Note that this is not the most general form that the algebra of the $\mathcal{D}_{A}$ and $\delta_{I}$ could have because the right hand side of (5.9) contains no term with a $\delta_{I}$ while the right hand side of (5.10) contains no term with a $\mathcal{D}_{A}$. Furthermore we impose that the $f_{I J}{ }^{K}$ and $g_{I A}{ }^{B}$ are constants (whereas the $T_{A B}{ }^{C}$ and $F_{A B}{ }^{I}$ are in general field dependent),

$$
f_{I J}{ }^{K}=\text { constant }, \quad g_{I A}{ }^{B}=\text { constant. }
$$


The conditions (4.19) read then for the various index pictures ${ }_{M N P}{ }^{Q}$ :

$$
\begin{aligned}
& I J K^{L}: \quad f_{I J}{ }^{M} f_{M K}{ }^{L}+f_{J K}{ }^{M} f_{M I}{ }^{L}+f_{K I}{ }^{M} f_{M J}{ }^{L}=0, \\
& { }_{I J K}{ }^{A}: \quad 0=0, \\
& I J A^{K}: \quad 0=0, \\
& { }_{I J A}{ }^{B}: \quad g_{I A}{ }^{C} g_{J C}{ }^{B}-g_{J A}{ }^{C} g_{I C}{ }^{B}=f_{I J}{ }^{K} g_{K A}{ }^{B}, \\
& { }_{I A B}{ }^{C}: \quad \delta_{I} T_{A B}{ }^{C}=-g_{I A}{ }^{D} T_{D B}{ }^{C}-g_{I B}{ }^{D} T_{A D}{ }^{C}+T_{A B}{ }^{D} g_{I D}{ }^{C} \text {, } \\
& I_{A B}{ }^{J}: \quad \delta_{I} F_{A B}{ }^{J}=-g_{I A}{ }^{C} F_{C B}{ }^{J}-g_{I B}{ }^{C} F_{A C}{ }^{J}-f_{I K}{ }^{J} F_{A B}{ }^{K}, \\
& A B C^{I}: \quad \sum_{A B C}\left(\mathcal{D}_{A} F_{B C}{ }^{I}+T_{A B}^{D} F_{D C}{ }^{I}\right)=0 \text {, } \\
& { }_{A B C}{ }^{D}: \quad \sum_{A B C}\left(\mathcal{D}_{A} T_{B C}{ }^{D}+T_{A B}{ }^{E} T_{E C}{ }^{D}+F_{A B}{ }^{I} g_{I C}{ }^{D}\right)=0 .
\end{aligned}
$$

(5.11) is the Jacobi identity for structure constants of Lie algebra. It just reflects that, according to (5.10), the $\delta_{I}$ are to form a Lie algebra with structure constants $f_{I J}{ }^{K}$. This Lie algebra is denoted by $\mathfrak{g}$ and chosen to be the direct sum of the Lorentz group and the Lie algebra $\mathfrak{g}_{\mathrm{YM}}$ of a YM gauge group, $\mathfrak{g}=\mathfrak{s o}(1,3) \oplus \mathfrak{g}_{\mathrm{YM}}$.

(5.14) imposes that the constants $g_{I A}{ }^{B}$ are the entries of matrices $g_{I}$ representing $\mathfrak{g}$ on the $\mathcal{D}$ 's because in matrix notation it reads just $\left[g_{I}, g_{J}\right]=f_{I J}{ }^{K} g_{K}$. To fulfill it, we choose the only nonvanishing $g_{I}$ to be those for the Lorentz algebra and, possibly, for two abelian elements $\delta_{(R)}, \delta_{(W)} \in \mathfrak{g}_{\mathrm{YM}}$ which belong to so-called $R$-transformations (these are $U(1)$-transformations which do not commute with SUSY transformations) and Weyl-transformations [Weyl-transformations are included here for the sake of generality; we shall drop them later again]:

$$
\begin{array}{lll}
{\left[l_{a b}, \mathcal{D}_{c}\right]=\eta_{c b} \mathcal{D}_{a}-\eta_{c a} \mathcal{D}_{b},} & {\left[l_{a b}, \mathcal{D}_{\alpha}\right]=-\sigma_{a b \alpha}{ }^{\beta} \mathcal{D}_{\beta},} & {\left[l_{a b}, \overline{\mathcal{D}}_{\dot{\alpha}}\right]=\bar{\sigma}_{a b} \dot{\beta} \dot{\alpha} \overline{\mathcal{D}}_{\dot{\beta}}} \\
{\left[\delta_{(R)}, \mathcal{D}_{a}\right]=0,} & {\left[\delta_{(R)}, \mathcal{D}_{\alpha}\right]=-\mathrm{i} \mathcal{D}_{\alpha},} & {\left[\delta_{(R)}, \overline{\mathcal{D}}_{\dot{\alpha}}\right]=\mathrm{i} \overline{\mathcal{D}}_{\dot{\alpha}}} \\
{\left[\delta_{(W)}, \mathcal{D}_{a}\right]=-\mathcal{D}_{a},} & {\left[\delta_{(W)}, \mathcal{D}_{\alpha}\right]=-\frac{1}{2} \mathcal{D}_{\alpha},} & {\left[\delta_{(W)}, \overline{\mathcal{D}}_{\dot{\alpha}}\right]=-\frac{1}{2} \overline{\mathcal{D}}_{\dot{\alpha}}}
\end{array}
$$

(5.15) and (5.16) require that the "torsions" $T_{A B}{ }^{C}$ and "curvatures" $F_{A B}{ }^{I}$ transform under $\mathfrak{g}$ according to linear representations characterized by their index pictures. They are thus fulfilled when the $T_{A B}{ }^{C}$ and $F_{A B}{ }^{I}$ are ordinary tensor fields with regard to the Lorentz group and the YM gauge group.

(5.17) and (5.18) are conditions on the $T_{A B}{ }^{C}$ and $F_{A B}{ }^{I}$ and their $\mathcal{D}_{A^{-}}$ transformations. They provide in particular in part the SUSY-transformations of these tensor fields (recall that the gauge transformations of a tensor fields are $\delta_{\hat{\xi}} T=\hat{\xi}^{M} \Delta_{M} T$ whose "SUSY-part" is thus $\hat{\xi}^{\alpha} \mathcal{D}_{\alpha} T+\overline{\hat{\xi}}_{\dot{\alpha}} \overline{\mathcal{D}}^{\dot{\alpha}} T$ ). (5.17) and (5.18) are called the Bianchi identities of $D=4, N=1$ SUGRA because they generalize the Bianchi identities of GR and YM theory (the latter are obtained from (5.17) for $A B C=a b c$ by setting all fields with spinors indices to zero). A set of tensor fields $\left\{T_{A B}{ }^{C}, F_{A B}{ }^{I}\right\}$ which satisfies these equations is called a "solution of the Bianchi identities". It was shown in [24] that the Bianchi identities (5.17) follow from (5.18) [using (4.16) and (5.15)].

Different solutions of the Bianchi identities lead to different formulations of $D=$ 4, $N=1$ SUGRA. However, two such 'different' formulations can actually still be 
equivalent because they may only differ by redefinitions of the fields or gauge parameters. Indeed, consider redefinitions of the gauge parameters of the form $\hat{\xi}^{M}=\hat{\xi}^{N} X_{N}^{M}$ where $X_{N}^{M}$ is a local invertible matrix whose entries are tensor fields. Such redefinitions of the gauge parameters correspond to redefinitions $\Delta_{M}^{\prime}=\left(X^{-1}\right)_{M}^{N} \Delta_{N}$ of the $\Delta^{\prime}$ 's (as these yield the same gauge transformations: on tensor fields one has $\hat{\xi}^{M} \Delta_{M} T=\hat{\xi}^{M} \Delta_{M}^{\prime} T$ for all tensor fields). Hence, two solutions of the Bianchi identities differing only by such redefinitions (which preserve (5.9) and (5.10) ) must be considered equivalent, since such redefinitions of gauge parameters can always be made in gauge theories (cf. section 2.5). By such redefinitions one can always achieve [25] that

$$
T_{\alpha \dot{\alpha}}^{a}=2 \mathrm{i} \sigma_{\alpha \dot{\alpha}}^{a}, \quad T_{\alpha \dot{\alpha}}^{\beta}=T_{\alpha \dot{\alpha}}^{\dot{\beta}}=T_{\alpha \beta}^{\gamma}=T_{\dot{\alpha} \dot{\beta}}^{\dot{\gamma}}=T_{a b}^{c}=F_{\alpha \dot{\alpha}}{ }^{i}=0 .
$$

Hence (5.20) can be assumed without loss of generality. These choices are therefore called "conventional constraints". The constraint $T_{a b}{ }^{c}=0$ determines the spin connection because (4.25) yields for $M=c$ :

$$
e_{\mu}^{a} e_{\nu}^{b} T_{a b}^{c}=\partial_{\mu} e_{\nu}^{c}-\partial_{\nu} e_{\mu}^{c}+\psi \frac{\beta}{\mu} \psi \underline{\alpha}_{\nu} T_{\underline{\alpha}}{ }^{c}+\left(e_{\mu}^{a} \psi \psi_{\nu}^{\alpha}-e_{\nu}^{a} \psi \underline{\alpha}^{\alpha}\right) T_{\underline{\alpha} a}^{c}+\left(e_{\mu}^{a} A_{\nu}^{I}-e_{\nu}^{a} A_{\mu}^{I}\right) g_{I a}{ }^{c}(5
$$

where $\left\{\psi_{\mu}^{\frac{\alpha}{\mu}}\right\}=\left\{\psi_{\mu}^{\alpha}, \bar{\psi}_{\mu}^{\dot{\alpha}}\right\}$ and summation convention as in (5.5). Using (5.19), the term $e_{\mu}^{a} A_{\nu}^{I} g_{I a}^{c}$ which occurs in (5.21) reads explicitly

$$
e_{\mu}^{a} A_{\nu}^{I} g_{I a}^{c}=\omega_{\nu \mu}^{c}+e_{\mu}^{c} A_{\nu}^{(W)} .
$$

Hence, for $T_{a b}{ }^{c}=0$ we obtain from (5.21):

$$
\omega_{[\mu \nu]}^{c}=\partial_{[\mu} e_{\nu]}^{c}+\frac{1}{2} \psi \frac{\beta}{\mu} \psi \frac{\alpha}{\nu} T_{\underline{\alpha} \underline{\beta}}^{c}+e_{[\mu}^{a} \psi \frac{\alpha}{\nu]} T_{\underline{\alpha} a}^{c}+e_{[\mu}^{c} A_{\nu]}^{(W)} .
$$

Note that this is analogous to (3.4) and determines $\omega_{\mu}^{a b}$ analogously to (3.5), using $\omega_{\mu \nu \rho}=\omega_{[\mu \nu] \rho}-\omega_{[\nu \rho] \mu}+\omega_{[\rho \mu] \nu}$.

Constraints in addition to (5.20) yield different off-shell formulations of $D=4$, $N=1$ SUGRA. The additional constraints cannot be arbitrarily chosen because the Bianchi identities (5.17) and (5.18) must be satisfied. The simplest solutions to the Bianchi identities are spelled out in the next subsections.

\subsection{Old minimal SUGRA}

We shall now present the so-called "old minimal" SUGRA theory which is certainly the most popular off-shell formulation of $D=4, N=1$ SUGRA. We shall start from the corresponding solution of the Bianchi identities (5.17) and (5.18) in presence of super-YM multiplets without discussing how one derives this solution systematically (for details see, e.g., [26]). Then we shall introduce chiral matter multiplets, spell out the gauge transformations and finally the construction of invariant actions, including the higher order invariants. 


\subsubsection{Old minimal solution of the Bianchi identities}

We shall present the solution for the case that $R$-transformations are possibly gauged (the version without gauged $R$-transformation is obtained simply by setting all fields with an index $(R)$ to zero), but without gauged Weyl-transformations,

$$
\delta_{(W)} \notin\left\{\delta_{i}\right\} .
$$

The torsions and curvatures are, except for those that can be obtained from the others using the graded symmetry in $A B$, or the following relations

$$
T_{\alpha a}{ }^{\beta}=-\left(T_{\dot{\alpha} a}^{\dot{\beta}}\right)^{*}, \quad T_{\alpha a}^{\dot{\beta}}=-\left(T_{\dot{\alpha} a}^{\beta}\right)^{*}, \quad F_{\alpha a}^{I}=\left(F_{\dot{\alpha} a}^{I}\right)^{*}, \quad F_{\alpha \beta}^{a b}=-\left(F_{\dot{\alpha} \dot{\beta}}^{a b}\right)^{*},
$$

or (4.25) (with $\left.T_{a b}^{c}=0\right)$ :

\begin{tabular}{c|c|c|c} 
& $A B=\dot{\alpha} b$ & $A B=\dot{\alpha} \dot{\beta}$ & $A B=\alpha \dot{\beta}$ \\
\hline$T_{A B}{ }^{c}$ & 0 & 0 & $2 \mathrm{i} \sigma_{\alpha \dot{\beta}}^{c}$ \\
$T_{A B}{ }^{\gamma}$ & $\frac{\mathrm{i}}{8} M \epsilon^{\gamma \alpha} \sigma_{b \alpha \dot{\alpha}}$ & 0 & 0 \\
$T_{A B}{ }^{\dot{\gamma}}$ & $-\mathrm{i}\left(\delta_{\dot{\alpha}}^{\dot{\gamma}} B_{b}+B^{c} \bar{\sigma}_{c b} \dot{\gamma} \dot{\alpha}\right)$ & 0 & 0 \\
$F_{A B}{ }^{i}$ & $\mathrm{i} \lambda^{i \alpha} \sigma_{b \alpha \dot{\alpha}}$ & 0 & 0 \\
$F_{A B}{ }^{c d}$ & $\mathrm{i} T^{c d \alpha} \sigma_{b \alpha \dot{\alpha}}-2 \mathrm{i} \sigma_{\alpha \dot{\alpha}}^{[c} T^{d]}{ }_{b}^{\alpha}$ & $-M \bar{\sigma}_{\dot{\alpha} \dot{\beta}}^{c d}$ & $2 \mathrm{i} \epsilon^{a b c d} \sigma_{a \alpha \dot{\beta}} B_{b}$
\end{tabular}

Here $M$ is a complex scalar field and $B_{a}$ is a real vector field. These fields are the auxiliary fields of the old minimal SUGRA multiplet [of course, that these fields are indeed auxiliary ones can not really be seen at this point but only from the action to be constructed later; however, one may anticipate it by counting the DOF off-shell and by inspecting the dimensions of these fields]. The $\lambda_{\alpha}^{i}$ are the fermions ("gauginos") of the super-YM multiplets, i.e., the "superpartners" of the YM gauge fields. Explicitly this yields:

$$
\begin{aligned}
& {\left[\mathcal{D}_{a}, \mathcal{D}_{b}\right]=-\frac{1}{2} F_{a b}{ }^{c d} l_{c d}-F_{a b}{ }^{i} \delta_{i}-T_{a b}{ }^{\alpha} \mathcal{D}_{\alpha}-T_{a b \dot{\alpha}} \overline{\mathcal{D}}^{\dot{\alpha}}} \\
& {\left[\mathcal{D}_{\alpha}, \mathcal{D}_{a}\right]=-\frac{1}{2} F_{\alpha a}{ }^{c d} l_{c d}+\mathrm{i} \sigma_{a \alpha \dot{\alpha}} \bar{\lambda}^{\dot{\alpha} i} \delta_{i}+\mathrm{i}\left(B_{a} \delta_{\alpha}^{\beta}-B^{b} \sigma_{b a \alpha}{ }^{\beta}\right) \mathcal{D}_{\beta}-\frac{i}{8} \bar{M} \sigma_{a \alpha \dot{\alpha}} \overline{\mathcal{D}}^{\dot{\alpha}}} \\
& {\left[\overline{\mathcal{D}}_{\dot{\alpha}}, \mathcal{D}_{a}\right]=-\frac{1}{2} F_{\dot{\alpha} a}{ }^{c d} l_{c d}-\mathrm{i} \sigma_{a \alpha \dot{\alpha}} \lambda^{\alpha i} \delta_{i}-\mathrm{i}\left(B_{a} \delta_{\dot{\alpha}}^{\dot{\beta}}+B^{b} \bar{\sigma}_{b a}{ }_{\dot{\alpha}}{ }_{\dot{\alpha}}\right) \overline{\mathcal{D}}_{\dot{\beta}}+\frac{\mathrm{i}}{8} M \sigma_{a \alpha \dot{\alpha}} \mathcal{D}^{\alpha}} \\
& {\left[\mathcal{D}_{\alpha}, \overline{\mathcal{D}}_{\dot{\alpha}}\right]=-2 \mathrm{i} \sigma_{\alpha \dot{\alpha}}^{a} \mathcal{D}_{a}-\mathrm{i} \epsilon^{a b c d} \sigma_{a \alpha \dot{\alpha}} B_{b} l_{c d}=-2 \mathrm{i} \mathcal{D}_{\alpha \dot{\alpha}}+2 B B_{\beta \dot{\alpha}} l_{\alpha}{ }^{\beta}-2 B_{\alpha \dot{\beta}} \bar{l}_{\dot{\alpha}} \dot{\beta}} \\
& {\left[\mathcal{D}_{\alpha}, \mathcal{D}_{\beta}\right]=\frac{1}{2} \bar{M} \sigma^{a b}{ }_{\alpha \beta} l_{a b}=\bar{M} l_{\alpha \beta}} \\
& {\left[\overline{\mathcal{D}}_{\dot{\alpha}}, \overline{\mathcal{D}}_{\dot{\beta}}\right]=\frac{1}{2} M \bar{\sigma}^{a b}{ }_{\dot{\alpha} \dot{\beta}} l_{a b}=-M \bar{l}_{\dot{\alpha} \dot{\beta}}}
\end{aligned}
$$

where $l_{\alpha \beta}$ and $l_{\dot{\alpha} \dot{\beta}}$ are the Lorentz $(\mathfrak{s l}(2, \mathbb{C}))$ generators acting on undotted and dotted spinor indices according to

$$
l_{\alpha \beta} X_{\gamma}=-\epsilon_{\gamma(\alpha} X_{\beta)}, \quad l_{\alpha \beta} \bar{X}_{\dot{\alpha}}=0, \quad \bar{l}_{\dot{\alpha} \dot{\beta}} \bar{X}_{\dot{\gamma}}=-\epsilon_{\dot{\gamma}(\dot{\alpha}} \bar{X}_{\dot{\beta})}, \quad \bar{l}_{\dot{\alpha} \dot{\beta}} X_{\alpha}=0 .
$$

They are related to the $l_{a b}$ by

$$
l_{a b}=\sigma_{a b}^{\alpha \beta} l_{\alpha \beta}-\bar{\sigma}_{a b}{ }^{\dot{\alpha} \dot{\beta}} \bar{l}_{\dot{\alpha} \dot{\beta}} .
$$


Furthermore the Bianchi identities yield

$$
\begin{aligned}
\mathcal{D}_{\alpha} M & =\frac{16}{3}\left(S_{\alpha}-\mathrm{i} \lambda_{\alpha}^{(R)}\right) \\
\mathcal{D}_{\alpha} \bar{M} & =0 \\
\mathcal{D}_{\alpha} B_{\beta \dot{\beta}} & =\frac{1}{3} \epsilon_{\beta \alpha}\left(\bar{S}_{\dot{\beta}}+4 \mathrm{i} \bar{\lambda}_{\dot{\beta}}^{(R)}\right)-\bar{U}_{\alpha \beta \dot{\beta}} \\
\mathcal{D}_{\alpha} \lambda_{\beta}^{i} & =\mathrm{i} \epsilon_{\alpha \beta} D^{i}+G_{\alpha \beta} \dot{i}^{i} \\
\mathcal{D}_{\alpha} \bar{\lambda}_{\dot{\alpha}}^{i} & =0 \\
\mathcal{D}_{\alpha} D^{i} & =\mathcal{D}_{\alpha \dot{\alpha}} \bar{\lambda}^{i \dot{\alpha}}+\frac{3 \mathrm{i}}{2} B_{\alpha \dot{\alpha}} \bar{\lambda}^{i \dot{\alpha}}
\end{aligned}
$$

where $D^{i}$ are real auxiliary fields of the super-YM multiplets and $S_{\alpha}, U_{\dot{\alpha} \dot{\beta} \gamma}$ and $G_{\alpha \beta}{ }^{i}$ are given by

$$
S_{\alpha}=T_{a b}{ }^{\beta} \sigma_{\alpha \beta}^{a b}, U_{\dot{\alpha} \dot{\beta} \gamma}=T_{a b \gamma} \bar{\sigma}_{\dot{\alpha} \dot{\beta}}^{a b}, W_{\alpha \beta \gamma}=T_{a b(\alpha} \sigma_{\beta \gamma)}^{a b}, G_{\alpha \beta}^{i}=-F_{a b}{ }^{i} \sigma_{\alpha \beta}^{a b} .
$$

Notice that the fields $D^{i}$ do not occur in any of the torsions or curvatures. They arise only 'indirectly' from the Bianchi identities because the latter determine $\mathcal{D}_{\alpha} \lambda_{\beta}^{i}$ only up to the piece which is antisymmetric in $\alpha$ and $\beta$ and purely imaginary. That piece is written as $\mathrm{i} \epsilon_{\alpha \beta} D^{i}$ which introduces thus additional fields $D^{i}$. That these fields are really needed, i.e., that they cannot be set to zero off-shell is then seen by imposing the algebra (5.24) on the $\lambda^{i}$ and $\bar{\lambda}^{i}$ with the result given in (5.32) (the right hand side of (5.32) does not vanish off-shell and therefore the $D^{i}$ cannot be set to zero off-shell either).

The tensor fields (5.33) arise when one decomposes $T_{a b}{ }^{\alpha}$ and $F_{a b}{ }^{i}$ into Lorentzirreducible parts by expressing them in terms of spinor indices (using $F_{\alpha \dot{\alpha} \beta \dot{\beta}}^{i}=$ $\sigma_{\alpha \dot{\alpha}}^{a} \sigma_{\beta \dot{\beta}}^{b} F_{a b}{ }^{i}$ etc) and then decomposing the resulting expressions into pieces which are totally symmetric in all undotted and all undotted spinor indices, respectively (splitting off $\epsilon$ 's):

$$
\begin{array}{ll} 
& T_{\alpha \dot{\alpha} \beta \dot{\beta} \gamma}=\epsilon_{\alpha \beta} U_{\dot{\alpha} \dot{\beta} \gamma}+\epsilon_{\dot{\beta} \dot{\alpha}}\left(W_{\alpha \beta \gamma}+\frac{2}{3} \epsilon_{\gamma(\alpha} S_{\beta)}\right) \\
\Leftrightarrow \quad & T_{a b \gamma}=\frac{1}{2} \bar{\sigma}_{a b} \dot{\alpha} \dot{\beta} U_{\dot{\alpha} \dot{\beta} \gamma}+\frac{1}{2} \sigma_{a b}{ }^{\alpha \beta} W_{\alpha \beta \gamma}-\frac{1}{3} \sigma_{a b \alpha \gamma} S^{\alpha}, \\
& F_{\alpha \dot{\alpha} \beta \dot{\beta}}{ }^{i}=\epsilon_{\alpha \beta} \bar{G}_{\dot{\alpha} \dot{\beta}}{ }^{i}+\epsilon_{\dot{\alpha} \dot{\beta}} G_{\alpha \beta}{ }^{i} \\
\Leftrightarrow \quad & F_{a b}{ }^{i}=\frac{1}{2} \bar{\sigma}_{a b} \dot{\alpha} \dot{\beta} \bar{G}_{\dot{\alpha} \dot{\beta}}{ }^{i}-\frac{1}{2} \sigma_{a b}{ }^{\alpha \beta} G_{\alpha \beta}{ }^{i} .
\end{array}
$$

For the sake of completeness, and for later use, let me also give the corresponding decomposition of the supercovariant version of the Riemann tensor $F_{a b}{ }^{c d}$ :

$$
\begin{aligned}
& F_{\alpha \dot{\alpha} \beta \dot{\beta} \gamma \dot{\gamma} \delta \dot{\delta}}=\epsilon_{\dot{\alpha} \dot{\beta}} \epsilon_{\dot{\gamma} \dot{\delta}}\left[X_{\alpha \beta \gamma \delta}-\frac{1}{6}\left(\epsilon_{\alpha \gamma} \epsilon_{\beta \delta}+\epsilon_{\beta \gamma} \epsilon_{\alpha \delta}\right) \mathcal{R}\right]-\epsilon_{\alpha \beta} \epsilon_{\dot{\gamma} \dot{\delta}} Y_{\gamma \delta \dot{\alpha} \dot{\beta}}+\text { c.c. } \\
& X_{\alpha \beta \gamma \delta}=\sigma_{(\alpha \beta}^{a b}{ }_{(\alpha \delta)} F_{a b c d}, \quad Y_{\alpha \beta \dot{\alpha} \dot{\beta}}=\bar{\sigma}_{\alpha b \dot{\beta}}^{a b} \sigma_{\alpha \beta} F_{a b c d}, \quad \mathcal{R}=F_{a b}{ }^{b a} .
\end{aligned}
$$

$X_{\alpha \beta \gamma \delta}, Y_{\alpha \beta \dot{\alpha} \dot{\beta}}$ and $\mathcal{R}$ are the supercovariant versions of the Weyl tensor, traceless Ricci tensor and Riemann curvature scalar, respectively (in spinor notation). I also note for later use another important result:

$$
\mathcal{D}^{\alpha} \mathcal{D}_{\alpha} M=\frac{8}{3} \mathcal{R}+\frac{32}{3} D^{(R)}+2 M \bar{M}-16 B^{a} B_{a}+16 \mathrm{i} \mathcal{D}_{a} B^{a} .
$$


Using the torsions in the table (5.23), one obtains from (5.22):

$$
\omega_{[\mu \nu]}^{a}=\partial_{[\mu} e_{\nu]}^{a}-2 \mathrm{i} \psi_{[\mu} \sigma^{a} \bar{\psi}_{\nu]} .
$$

This is precisely the same expression as (3.4). Hence the spin connection of the old minimal formulation is given again by (3.5).

\subsubsection{Chiral matter multiplets}

Next we discuss so-called chiral matter multiplets. These consist of tensorial matter fields $\varphi, \chi_{\alpha}, F$ where $\varphi$ and $F$ are complex scalar fields and $\chi_{\alpha}$ are Weyl spinor fields. These fields may carry additional indices which refer to the YM gauge group (more precisely, a representation thereof), which we shall suppress. So, one should think of $\varphi$ as a column vector on which representation matrices $T_{i}$ of the YM-Lie algebra $\mathfrak{g}_{\mathrm{YM}}$ act, and the same applies to $\chi_{\alpha}$ and $F$. These representation matrices $T_{i}$ agree on $\varphi, \chi_{\alpha}, F$ for all $i$ except for $i=(R)$ (this exception will become clear below),

$$
\begin{array}{ll}
i \neq(R): \quad & \delta_{i} \phi=-T_{i} \phi, \delta_{i} \chi_{\alpha}=-T_{i} \chi_{\alpha}, \delta_{i} F=-T_{i} F \\
i=(R): \quad & \delta_{(R)} \varphi=-T_{(R)} \varphi, \delta_{(R)} \chi_{\alpha}=-\left(T_{(R)}+\mathrm{i}\right) \chi_{\alpha}, \delta_{(R)} F=-\left(T_{(R)}+2 \mathrm{i}\right) F \\
& {\left[T_{i}, T_{j}\right]=f_{i j}{ }^{k} T_{k} .}
\end{array}
$$

The Lorentz group acts on $\varphi, \chi_{\alpha}, F$ in the standard way,

$$
l_{a b} \varphi=0, \quad l_{a b} \chi_{\alpha}=-\left(\sigma_{a b} \chi\right)_{\alpha}, \quad l_{a b} F=0 .
$$

Then (5.9), (5.10) are satisfied on the $\varphi, \chi_{\alpha}, F$. (5.24) is satisfied with the following transformations:

$$
\begin{array}{ll}
\mathcal{D}_{\alpha} \varphi=\chi_{\alpha}, & \overline{\mathcal{D}}_{\dot{\alpha}} \varphi=0, \\
\mathcal{D}_{\alpha} \chi_{\beta}=-\epsilon_{\alpha \beta} F, & \overline{\mathcal{D}}_{\dot{\alpha}} \chi_{\alpha}=-2 \mathrm{i} \sigma_{\alpha \dot{\alpha}}^{a} \mathcal{D}_{a} \varphi, \\
\mathcal{D}_{\alpha} F=-\frac{1}{2} \bar{M} \chi_{\alpha}, & \overline{\mathcal{D}}_{\dot{\alpha}} F=-2 \mathrm{i} \mathcal{D}_{\alpha \dot{\alpha}} \chi^{\alpha}-4 \bar{\lambda}_{\dot{\alpha}}^{i} \delta_{i} \varphi+B_{\alpha \dot{\alpha}} \chi^{\alpha} .
\end{array}
$$

This explains in particular the relations for $\delta_{(R)}$ in (5.39), as $\mathcal{D}_{\alpha}$ carries $R$-weight 1 , cf. (5.19).

The field content of chiral matter multiplets and the transformations (5.41) can be found as follows. We start just with the field $\varphi$, which is chosen to be the "lowest" component field of the multiplet to be constructed (i.e., it has lowest dimension). We impose $\overline{\mathcal{D}}_{\dot{\alpha}} \varphi=0$ which may be viewed as the simplest possible $\overline{\mathcal{D}}_{\dot{\alpha}}$-transformation one may choose 6 (that choice is possible because (5.24) requires $\left[\overline{\mathcal{D}}_{\dot{\alpha}}, \overline{\mathcal{D}}_{\dot{\beta}}\right] \varphi=\frac{1}{2} M \bar{\sigma}^{a b}{ }_{\dot{\alpha} \dot{\beta}} l_{a b} \varphi$ which vanishes owing to $\left.l_{a b} \varphi=0\right)$. $\mathcal{D}_{\alpha} \varphi$ is then defined to be a new field denoted by $\chi_{\alpha}$ which thus becomes the second member of the multiplet.

We have thus fixed the $\mathcal{D}_{\underline{\alpha}}$-transformations of $\varphi$ (and also of $\bar{\varphi}$ by complex conjugation) and introduced new fields $\chi_{\alpha}$. Next we have to define the transformations of these fields. Let us first consider $\mathcal{D}_{\alpha} \chi_{\beta}$. Using $\chi_{\beta}=\mathcal{D}_{\beta} \varphi$ we obtain

$$
\mathcal{D}_{\alpha} \chi_{\beta}=\mathcal{D}_{\alpha} \mathcal{D}_{\beta} \varphi=\frac{1}{2}\left(\mathcal{D}_{\alpha} \mathcal{D}_{\beta}+\mathcal{D}_{\beta} \mathcal{D}_{\alpha}\right) \varphi+\frac{1}{2}\left(\mathcal{D}_{\alpha} \mathcal{D}_{\beta}-\mathcal{D}_{\beta} \mathcal{D}_{\alpha}\right) \varphi
$$

\footnotetext{
${ }^{6}$ In accordance with standard SUSY terminology, $\overline{\mathcal{D}}_{\dot{\alpha}}$-invariant fields are called "chiral fields". Hence, $\varphi$ is a chiral field and that explains why the whole multiplet is termed "chiral multiplet".
} 
Up to the factor $1 / 2$, the first term on the right hand side is the graded commutator $\left[\mathcal{D}_{\alpha}, \mathcal{D}_{\beta}\right]$ (since $\mathcal{D}_{\alpha}$ and $\mathcal{D}_{\beta}$ are Grassmann odd their graded commutator is the anticommutator). (5.24) imposes that this term must vanish (owing to $l_{a b} \varphi=0$ ). The second term is antisymmetric in $\alpha$ and $\beta$ and thus proportional to $\epsilon_{\alpha \beta}$. We define it to be $-\epsilon_{\alpha \beta} F$ where $F$ is a new field (an additional member of the multiplet). This yields the transformations $\mathcal{D}_{\alpha} \chi_{\beta}=-\epsilon_{\alpha \beta} F$ in (5.41). To define $\overline{\mathcal{D}}_{\dot{\alpha}} \chi_{\alpha}$ we proceed similarly:

$$
\overline{\mathcal{D}}_{\dot{\alpha}} \chi_{\alpha}=\overline{\mathcal{D}}_{\dot{\alpha}} \mathcal{D}_{\alpha} \varphi=\left(\overline{\mathcal{D}}_{\dot{\alpha}} \mathcal{D}_{\alpha}+\mathcal{D}_{\alpha} \overline{\mathcal{D}}_{\dot{\alpha}}\right) \varphi-\mathcal{D}_{\alpha} \overline{\mathcal{D}}_{\dot{\alpha}} \varphi
$$

The first term on the right hand side is the graded commutator $\left[\mathcal{D}_{\alpha}, \overline{\mathcal{D}}_{\dot{\alpha}}\right] \varphi$. According to (5.24) it should be equal to $-2 \mathrm{i} \sigma_{\alpha \dot{\alpha}}^{a} \mathcal{D}_{a} \varphi$ (owing to $l_{a b} \varphi=0$ ). The second term must vanish because of $\overline{\mathcal{D}}_{\dot{\alpha}} \varphi=0$. This yields the transformations $\overline{\mathcal{D}}_{\dot{\alpha}} \chi_{\alpha}=-2 \mathrm{i} \sigma_{\alpha \dot{\alpha}}^{a} \mathcal{D}_{a} \varphi$ in (5.41). Note that this really defines $\overline{\mathcal{D}}_{\dot{\alpha}} \chi_{\alpha}$ completely because, using (5.6), we obtain:

$$
\begin{aligned}
\mathcal{D}_{a} \varphi & =E_{a}^{\mu}\left(\partial_{\mu}-\psi_{\mu}^{\alpha} \mathcal{D}_{\alpha}-\bar{\psi}_{\mu \dot{\alpha}} \overline{\mathcal{D}}^{\dot{\alpha}}-\frac{1}{2} \omega_{\mu}{ }^{a b} l_{a b}-A_{\mu}^{i} \delta_{i}\right) \varphi \\
& =E_{a}^{\mu}\left(\partial_{\mu} \varphi-\psi_{\mu}^{\alpha} \chi_{\alpha}-A_{\mu}^{i} \delta_{i} \varphi\right) .
\end{aligned}
$$

As we have introduced a new field $F$, we must now determine its transformations. $\mathcal{D}_{\alpha} \chi_{\beta}=-\epsilon_{\alpha \beta} F$ gives $2 F=\mathcal{D}_{\beta} \chi^{\beta}$. Using this, we obtain

$$
\mathcal{D}_{\alpha} F=\frac{1}{2} \mathcal{D}_{\alpha} \mathcal{D}_{\beta} \chi^{\beta}=\frac{1}{2}\left[\mathcal{D}_{\alpha}, \mathcal{D}_{\beta}\right] \chi^{\beta}-\frac{1}{2} \mathcal{D}_{\beta} \mathcal{D}_{\alpha} \chi^{\beta} .
$$

Using the algebra (5.24), we obtain for the first term on the right hand side of (5.43):

$$
\frac{1}{2}\left[\mathcal{D}_{\alpha}, \mathcal{D}_{\beta}\right] \chi^{\beta}=\frac{1}{2} \bar{M} l_{\alpha \beta} \chi^{\beta}=-\frac{3}{4} \bar{M} \chi_{\alpha} .
$$

Using once again $\mathcal{D}_{\alpha} \chi_{\beta}=-\epsilon_{\alpha \beta} F$, the second term on the right hand side of (5.43) is:

$$
-\frac{1}{2} \mathcal{D}_{\beta} \mathcal{D}_{\alpha} \chi^{\beta}=-\frac{1}{2} \mathcal{D}_{\beta}\left(\delta_{\alpha}^{\beta} F\right)=-\frac{1}{2} \mathcal{D}_{\alpha} F
$$

Bringing this term to the left hand side of (5.43) we obtain the transformation $\mathcal{D}_{\alpha} F=$ $-\frac{1}{2} \bar{M} \chi_{\alpha}$ in (5.41). Finally we compute $\overline{\mathcal{D}}_{\dot{\alpha}} F$ starting again from $2 F=\mathcal{D}_{\beta} \chi^{\beta}$ and then using the results for $\overline{\mathcal{D}}_{\dot{\alpha}} \chi^{\alpha}$ and $\mathcal{D}_{\alpha} \varphi$ :

$$
\begin{aligned}
\overline{\mathcal{D}}_{\dot{\alpha}} F & =\frac{1}{2} \overline{\mathcal{D}}_{\dot{\alpha}} \mathcal{D}_{\alpha} \chi^{\alpha} \\
& =\frac{1}{2}\left[\overline{\mathcal{D}}_{\dot{\alpha}}, \mathcal{D}_{\alpha}\right] \chi^{\alpha}-\frac{1}{2} \mathcal{D}_{\alpha} \overline{\mathcal{D}}_{\dot{\alpha}} \chi^{\alpha} \\
& =\frac{1}{2}\left[\overline{\mathcal{D}}_{\dot{\alpha}}, \mathcal{D}_{\alpha}\right] \chi^{\alpha}+\mathrm{i} \sigma^{a \alpha}{ }_{\dot{\alpha}} \mathcal{D}_{\alpha} \mathcal{D}_{a} \varphi \\
& =\frac{1}{2}\left[\overline{\mathcal{D}}_{\dot{\alpha}}, \mathcal{D}_{\alpha}\right] \chi^{\alpha}+\mathrm{i} \sigma^{a \alpha}{ }_{\dot{\alpha}}\left[\mathcal{D}_{\alpha}, \mathcal{D}_{a}\right] \varphi+\mathrm{i} \sigma^{a \alpha}{ }_{\dot{\alpha}} \mathcal{D}_{a} \mathcal{D}_{\alpha} \varphi \\
& =\frac{1}{2}\left[\overline{\mathcal{D}}_{\dot{\alpha}}, \mathcal{D}_{\alpha}\right] \chi^{\alpha}+\mathrm{i} \sigma^{a \alpha}{ }_{\dot{\alpha}}\left[\mathcal{D}_{\alpha}, \mathcal{D}_{a}\right] \varphi+\mathrm{i} \sigma_{\dot{\alpha}}^{a \alpha}{ }_{\dot{\alpha}} \mathcal{D}_{a} \chi_{\alpha}
\end{aligned}
$$

$\left[\overline{\mathcal{D}}_{\dot{\alpha}}, \mathcal{D}_{\alpha}\right] \chi^{\alpha}$ and $\sigma_{\alpha \alpha}^{a \alpha}\left[\mathcal{D}_{\alpha}, \mathcal{D}_{a}\right] \varphi$ can be worked out using the algebra (5.24): the former yields terms proportional to $\mathcal{D}_{\alpha \dot{\alpha}} \chi^{\alpha}$ and $B_{\alpha \dot{\alpha}} \chi^{\alpha}$, the latter terms proportional to $\bar{\lambda}_{\dot{\alpha}}^{i} \delta_{i} \varphi$ and $B_{\alpha \dot{\alpha}} \chi^{\alpha}$. Working out the precise coefficients one obtains the result for $\overline{\mathcal{D}}_{\dot{\alpha}} F$ given in (5.41). This time we did not introduce any new field and therefore this ends the derivation of the multiplet and the transformations (5.41). The fields $F$ are the auxiliary fields of the chiral matter multiplets. 


\subsubsection{Gauge transformations}

We can now spell out the gauge transformations of old minimal SUGRA coupled to super-YM multiplets and chiral matter multiplets with field content

$$
e_{\mu}^{a}, \psi_{\mu}^{\alpha}, M, B_{a} ; \quad A_{\mu}^{i}, \lambda_{\alpha}^{i}, D^{i} ; \quad \varphi, \chi_{\alpha}, F .
$$

$\left(e_{\mu}^{a}, \psi_{\mu}^{\alpha}, M, B_{a}\right)$ is called the old minimal SUGRA multiplet, $\left(A_{\mu}^{i}, \lambda_{\alpha}^{i}, D^{i}\right)$ the super-YM multiplet(s), $\left(\varphi, \chi_{\alpha}, F\right)$ the chiral matter multiplet(s). The gauge transformations of $M$, $B_{a}, D^{i}, \varphi, \chi_{\alpha}$ and $F$ are obtained from (4.1) using (5.27) through (5.32) and (5.41) and their complex conjugates, the gauge transformations of the gauge fields from (4.22) using the torsions and curvatures of old minimal SUGRA:

$$
\begin{aligned}
& \delta_{\hat{\xi}} e_{\mu}^{a}=\partial_{\mu} \hat{\xi}^{a}+\frac{1}{2}\left(e_{\mu}^{b} \hat{\xi}^{c d}-\omega_{\mu}^{c d} \hat{\xi}^{b}\right) g_{[c d] b}{ }^{a}+\psi{ }^{\alpha} \hat{\xi}^{\underline{\beta}} T_{\underline{\beta}} \underline{\alpha}^{a} \\
& =\partial_{\mu} \hat{\xi}^{a}-\omega_{\mu b}{ }^{a} \hat{\xi}^{b}+\hat{\xi}_{b}^{a} e_{\mu}^{b}+2 \mathrm{i} \hat{\xi} \sigma^{a} \bar{\psi}_{\mu}-2 \mathrm{i} \psi_{\mu} \sigma^{a} \overline{\hat{\xi}} \\
& \delta_{\hat{\xi}} \psi_{\mu}^{\alpha}=\partial_{\mu} \hat{\xi}^{\alpha}+\frac{1}{2}\left(\psi_{\mu}^{\beta} \hat{\xi}^{a b}-\omega_{\mu}^{a b} \hat{\xi}^{\beta}\right) g_{[a b] \beta}{ }^{\alpha}+\left(\psi_{\mu}^{\beta} \hat{\xi}^{(R)}-A_{\mu}^{(R)} \hat{\xi}^{\beta}\right) g_{(R) \beta}{ }^{\alpha} \\
& +\left(e_{\mu}^{a} \hat{\xi} \underline{\beta}-\psi \frac{\beta}{\mu} \hat{\xi}^{a}\right) T_{\underline{\beta} a}{ }^{\alpha}+e_{\mu}^{a} \hat{\xi}^{b} T_{b a}{ }^{\alpha} \\
& =\partial_{\mu} \hat{\xi}^{\alpha}-\frac{1}{2} \omega_{\mu}^{a b}\left(\hat{\xi} \sigma_{a b}\right)^{\alpha}-\mathrm{i} A_{\mu}^{(R)} \hat{\xi}^{\alpha}+\frac{1}{2} \hat{\xi}^{a b}\left(\psi_{\mu} \sigma_{a b}\right)^{\alpha}+\mathrm{i} \psi_{\mu}^{\alpha} \hat{\xi}^{(R)} \\
& +\left(e_{\mu}^{a} \hat{\xi}^{\beta}-\psi_{\mu}^{\beta} \hat{\xi}^{a}\right) T_{\beta a}{ }^{\alpha}-\left(e_{\mu}^{a} \overline{\hat{\xi}}^{\dot{\beta}}-\bar{\psi}_{\mu}^{\dot{\beta}} \hat{\xi}^{a}\right) T_{\dot{\beta} a}{ }^{\alpha}+e_{\mu}^{a} \hat{\xi}^{b} T_{b a}{ }^{\alpha} \\
& \delta_{\hat{\xi}} M=\hat{\xi}^{a} \mathcal{D}_{a} M+\frac{16}{3} \hat{\xi}^{\alpha}\left(S_{\alpha}-\mathrm{i} \lambda_{\alpha}^{(R)}\right)+2 \mathrm{i} \hat{\xi}^{(R)} M \\
& \delta_{\hat{\xi}} B_{\alpha \dot{\alpha}}=\hat{\xi}^{b} \mathcal{D}_{b} B_{a}-\sigma_{\alpha \dot{\alpha}}^{a} \hat{\xi}_{a}^{b} B_{b}+\left[-\frac{1}{3} \hat{\xi}_{\alpha}\left(\bar{S}_{\dot{\alpha}}+4 \mathrm{i} \bar{\lambda}_{\dot{\alpha}}^{(R)}\right)+\hat{\xi}^{\beta} \bar{U}_{\beta \alpha \dot{\alpha}}+\text { c.c. }\right] \\
& \delta_{\hat{\xi}} A_{\mu}^{i}=\partial_{\mu} \hat{\xi}^{i}-A_{\mu}^{j} \hat{\xi}^{k} f_{k j}{ }^{i}+\left(\psi \frac{\alpha}{\mu} \hat{\xi}^{a}-e_{\mu}^{a} \hat{\xi}^{\underline{\alpha}}\right) F_{a \underline{\alpha}}{ }^{i}+e_{\mu}^{a} \hat{\xi}^{b} F_{b a}{ }^{i} \\
& =\partial_{\mu} \hat{\xi}^{i}-A_{\mu}^{j} \hat{\xi}^{k} f_{k j}{ }^{i}-\mathrm{i} \hat{\xi} \sigma_{\mu} \bar{\lambda}^{i}+\mathrm{i} \lambda^{i} \sigma_{\mu} \overline{\hat{\xi}} \\
& +\mathrm{i} \hat{\xi}^{a}\left(\psi_{\mu} \sigma_{a} \bar{\lambda}^{i}-\lambda^{i} \sigma_{a} \bar{\psi}_{\mu}\right)+e_{\mu}^{a} \hat{\xi}^{b} F_{b a}{ }^{i} \\
& \delta_{\hat{\xi}} \lambda_{\alpha}^{i}=\hat{\xi}^{a} \mathcal{D}_{a} \lambda_{\alpha}^{i}-\frac{1}{2} \hat{\xi}^{a b}\left(\sigma_{a b} \lambda^{i}\right)_{\alpha}+\hat{\xi}^{j} \lambda^{k} f_{k j}{ }^{i}+\mathrm{i} \hat{\xi}^{(R)} \lambda_{\alpha}^{i}-\mathrm{i} \hat{\xi}_{\alpha} D^{i}+\xi^{\beta} G_{\beta \alpha}{ }^{i} \\
& \delta_{\hat{\xi}} D^{i}=\hat{\xi}^{a} \mathcal{D}_{a} D^{i}+\hat{\xi}^{j} D^{k} f_{k j}{ }^{i}+\left(\hat{\xi}^{\alpha} \mathcal{D}_{\alpha \dot{\alpha}} \bar{\lambda}^{i \dot{\alpha}}+\frac{3 \mathrm{i}}{2} \hat{\xi}^{\alpha} B_{\alpha \dot{\alpha}} \bar{\lambda}^{i \dot{\alpha}}+\text { c.c. }\right) \\
& \delta_{\hat{\xi}} \varphi=\hat{\xi}^{a} \mathcal{D}_{a} \varphi+\hat{\xi}^{i} \delta_{i} \varphi+\hat{\xi} \chi \\
& \delta_{\hat{\xi}} \chi_{\alpha}=\hat{\xi}^{a} \mathcal{D}_{a} \chi_{\alpha}-\frac{1}{2} \hat{\xi}^{a b}\left(\sigma_{a b} \chi\right)_{\alpha}+\hat{\xi}^{i} \delta_{i} \chi_{\alpha}+\hat{\xi}_{\alpha} F+2 \mathrm{i}\left(\sigma^{a} \overline{\hat{\xi}}\right)_{\alpha} \mathcal{D}_{a} \varphi \\
& \delta_{\hat{\xi}} F=\hat{\xi}^{a} \mathcal{D}_{a} F+\hat{\xi}^{i} \delta_{i} F-\frac{1}{2} \chi \hat{\xi} \bar{M}-2 \mathrm{i} \mathcal{D}_{a} \chi \sigma^{a} \overline{\hat{\xi}}-4 \bar{\lambda}^{i} \overline{\hat{\xi}} \delta_{i} \varphi+B_{a} \chi \sigma^{a} \overline{\hat{\xi}} .
\end{aligned}
$$

The gauge transformation (5.45) of the vierbein agrees entirely with the transformation given in equation (3.18). The gauge transformations (5.46) of the gravitino involve the torsions $T_{\beta a}{ }^{\alpha}, T_{\dot{\beta} a}{ }^{\alpha}$ given in table (5.23), and $T_{b a}{ }^{\alpha}$ obtained from (4.25). If one sets $M, B_{a}, A_{\mu}^{(R)}$ and $\hat{\xi}^{(R)}$ to zero, $T_{a b}{ }^{\alpha}$ reduces to $E_{a}^{\mu} E_{b}^{\nu}\left(\nabla_{\mu} \psi_{\nu}^{\alpha}-\nabla_{\nu} \psi_{\mu}^{\alpha}\right)$ and the whole expression (5.46) collapses to the transformation given in equation (3.27). This reflects that the auxiliary fields $M$ and $B_{a}$ vanish on-shell in the off-shell formulation of pure $D=4, N=1$ SUGRA when $R$-transformations are not gauged, as we shall see below.

Let us also indicate how the transformations (5.45) through (5.54) read in terms of the parameters $\xi$. According to (4.31) the transformations of $M, B_{a}, \lambda_{\alpha}^{i}, D^{i}, \varphi, \chi_{\alpha}, F$ are 
obtained from those given above simply by the replacements $\hat{\xi}^{a} \mathcal{D}_{a} \rightarrow \xi^{\mu} \partial_{\mu}, \hat{\xi^{\underline{\alpha}}} \rightarrow \xi^{\underline{\alpha}}$, $\hat{\xi}^{a b} \rightarrow \xi^{a b}, \hat{\xi}^{i} \rightarrow \xi^{i}$. For the transformations of the gauge fields one obtains from (4.32) and (4.33):

$$
\begin{aligned}
\delta_{\xi} e_{\mu}^{a}= & \xi^{\nu} \partial_{\nu} e_{\mu}^{a}+\partial_{\mu} \xi^{\nu} e_{\nu}^{a}+\xi_{b}^{a} e_{\mu}^{b}+2 \mathrm{i} \xi \sigma^{a} \bar{\psi}_{\mu}-2 \mathrm{i} \psi_{\mu} \sigma^{a} \bar{\xi} \\
\delta_{\xi} \psi_{\mu}^{\alpha}= & \xi^{\nu} \partial_{\nu} \psi_{\mu}^{\alpha}+\partial_{\mu} \xi^{\nu} \psi_{\nu}^{\alpha}+\partial_{\mu} \xi^{\alpha}-\frac{1}{2} \omega_{\mu}^{a b}\left(\xi \sigma_{a b}\right)^{\alpha}-\mathrm{i} A_{\mu}^{(R)} \xi^{\alpha} \\
& +\frac{1}{2} \xi^{a b}\left(\psi_{\mu} \sigma_{a b}\right)^{\alpha}+\mathrm{i} \psi_{\mu}^{\alpha} \xi^{(R)}+e_{\mu}^{a} \xi^{\beta} T_{\beta a}{ }^{\alpha}-e_{\mu}^{a} \bar{\xi}^{\dot{\beta}} T_{\dot{\beta} a}{ }^{\alpha} \\
= & \xi^{\nu} \partial_{\nu} \psi_{\mu}^{\alpha}+\partial_{\mu} \xi^{\nu} \psi_{\nu}^{\alpha}+\frac{1}{2} \xi^{a b}\left(\psi_{\mu} \sigma_{a b}\right)^{\alpha}+\mathrm{i} \psi_{\mu}^{\alpha} \xi^{(R)} \\
& +\nabla_{\mu} \xi^{\alpha}-\mathrm{i} \xi^{\alpha} B_{\mu}+\mathrm{i} B^{\nu}\left(\xi \sigma_{\nu \mu}\right)^{\alpha}+\frac{\mathrm{i}}{8} M\left(\bar{\xi} \bar{\sigma}_{\mu}\right)^{\alpha} \\
\delta_{\xi} A_{\mu}^{i}= & \xi^{\nu} \partial_{\nu} A_{\mu}^{i}+\partial_{\mu} \xi^{\nu} A_{\nu}^{i}+\partial_{\mu} \xi^{i}-A_{\mu}^{j} \xi^{k} f_{k j}{ }^{i}-\mathrm{i} \xi \sigma_{\mu} \bar{\lambda}^{i}+\mathrm{i} \lambda^{i} \sigma_{\mu} \bar{\xi}
\end{aligned}
$$

\subsubsection{Action}

It was proved in [25, 27] that the most general local function invariant up to a total divergence under the gauge transformations given in section 5.3 .3 is, up to a total divergence:

$$
\begin{aligned}
L_{\text {old }} & =e\left(\overline{\mathcal{D}}^{2}-4 \mathrm{i} \psi_{\mu} \sigma^{\mu} \overline{\mathcal{D}}-3 M+16 \psi_{\mu} \sigma^{\mu \nu} \psi_{\nu}\right) \mathcal{A}+\text { c.c. } \\
\mathcal{A} & =P(\bar{W}, \bar{\lambda}, \bar{\varphi})+\left(\mathcal{D}^{2}-\bar{M}\right) \Omega(T)
\end{aligned}
$$

where $\bar{W}_{\dot{\alpha} \dot{\beta} \dot{\gamma}}$ is the complex conjugate of $W_{\alpha \beta \gamma}$ in (5.33), $\overline{\mathcal{D}}^{2}$ and $\mathcal{D}^{2}$ are shorthand notations for $\overline{\mathcal{D}}_{\dot{\alpha}} \overline{\mathcal{D}}^{\dot{\alpha}}$ and $\mathcal{D}^{\alpha} \mathcal{D}_{\alpha}$ respectively,

$$
\mathcal{D}^{2}=\mathcal{D}^{\alpha} \mathcal{D}_{\alpha}, \quad \overline{\mathcal{D}}^{2}=\overline{\mathcal{D}}_{\dot{\alpha}} \overline{\mathcal{D}}^{\dot{\alpha}},
$$

$\Omega$ is invariant under all $\delta_{I}, P$ is invariant under all $\delta_{I}$ except under $R$-transformations and has $R$-weight 2 ,

$$
\delta_{I} \Omega=0 \quad \forall I, \quad \delta_{I} P=0 \quad \forall I \neq(R), \quad \delta_{(R)} P=-2 \mathrm{i} P .
$$

Of course the conditions imposed by $\delta_{(R)}$ are present only if we require $R$-invariance. The invariance of (5.58) under local SUSY transformations up to a total divergence is explicitly demonstrated in appendix B.2 (the invariance under the remaining gauge transformations is evident). I emphasize that $P$, as indicated by its arguments, depends only on the $\bar{W}_{\dot{\alpha} \dot{\beta} \dot{\gamma}}, \bar{\lambda}_{\dot{\alpha}}^{i}$ and $\bar{\varphi}$ but no (covariant) derivatives thereof. In contrast, $\Omega$ is an arbitrary function of the tensor fields only subject to (5.59). Let us now spell out various contributions to the Lagrangian obtained from (5.58).

Pure SUGRA action. The off-shell version of the pure SUGRA action arises when $\mathcal{A}$ is proportional to $\bar{M}$ (i.e., $P=0$ and $\Omega=$ constant). Then (5.27) and (5.37) (resp. their complex conjugates) yield straightforwardly:

$$
\begin{aligned}
& \mathcal{A}=\frac{3}{32} \bar{M} \quad \Rightarrow \\
& \begin{array}{r}
L_{\text {old }}=e\left[\frac{1}{2} \mathcal{R}-2 \mathrm{i} \psi_{\mu} \sigma^{\mu}\left(\bar{S}+\mathrm{i} \bar{\lambda}^{(R)}\right)+2 \mathrm{i}\left(S-\mathrm{i} \lambda^{(R)}\right) \sigma^{\mu} \bar{\psi}_{\mu}+2 D^{(R)}\right. \\
\left.\quad-3 B_{a} B^{a}-\frac{3}{16} M \bar{M}+\frac{3}{2}\left(\bar{M} \psi_{\mu} \sigma^{\mu \nu} \psi_{\nu}+M \bar{\psi}_{\mu} \bar{\sigma}^{\mu \nu} \bar{\psi}_{\nu}\right)\right]
\end{array}
\end{aligned}
$$


where $\lambda^{(R)}$ and $D^{(R)}$ contribute of course only if $R$-transformations are gauged - otherwise these fields simply have to be set to zero. In fact the Lagrangian (5.60) by itself is inconsistent in presence of these fields as one sees, for instance, from the EOM for $D^{(R)}$ which would read $2 e=0$. This is cured when the YM Lagrangian $L_{Y M}$ given below is added as it contains terms which are quadratic and of higher order in $A_{\mu}^{(R)}, \lambda^{(R)}$ and $D^{(R)}$. The locally $R$-symmetric SUGRA Lagrangian was first constructed in 28 . When $R$-transformations are not gauged, (5.60) reduces to the old minimal version of the pure SUGRA action (3.3) as given first in [20, 21]:

$$
L_{\text {pure }}=e\left(\frac{1}{2} R-3 B_{a} B^{a}-\frac{3}{16} M \bar{M}\right)+2 \epsilon^{\mu \nu \rho \sigma}\left(\nabla_{\mu} \psi_{\nu} \sigma_{\rho} \bar{\psi}_{\sigma}+\psi_{\sigma} \sigma_{\rho} \nabla_{\mu} \bar{\psi}_{\nu}\right)
$$

with $R=E_{b}^{\mu} E_{a}^{\nu} R_{\mu \nu}^{a b}$ as in (3.3). (15.61) arises from (5.60) by working out the supercovariant tensor fields $\mathcal{R}$ and $S_{\alpha}$ explicitly. For instance, the supercovariant curvature scalar $\mathcal{R}$ contains gravitino dependent contributions that combine with the term $2 \mathrm{i} S \sigma^{\mu} \bar{\psi}_{\mu}+$ c.c. to the familiar kinetic term for the gravitino in (5.61). Furthermore, the terms linear in $B, M$ and $\bar{M}$, i.e. those contained in $\mathcal{R}, S$ and $\bar{S}$ and the last two terms in (5.60), cancel out exactly. Notice that the EOM deriving from (5.61) set indeed both $M$ and $B_{a}$ to zero.

Locally supersymmetric YM action. The locally supersymmetric YM Lagrangian arises from the contribution $\frac{1}{16} \bar{\lambda}^{i} \bar{\lambda}_{i}$ to $P$ (nonabelian indices $i$ are lowered with the Cartan-Killing metric of the Yang-Mills gauge group and Abelian ones with the unit matrix). It reads

$$
\begin{array}{r}
e^{-1} L_{Y M}=-\frac{1}{4} F_{\mu \nu}{ }^{i} F^{\mu \nu}{ }_{i}-\frac{\mathrm{i}}{2}\left(\lambda^{i} \sigma^{\mu} \nabla_{\mu} \bar{\lambda}_{i}+\bar{\lambda}^{i} \bar{\sigma}^{\mu} \nabla_{\mu} \lambda_{i}\right)+\frac{1}{2} D^{i} D_{i}+\frac{3}{2} \lambda^{i} \sigma^{\mu} \bar{\lambda}_{i} B_{\mu} \\
-\frac{1}{2} e^{-1} F_{\mu \nu}{ }^{i} \epsilon^{\mu \nu \rho \sigma}\left(\psi_{\rho} \sigma_{\sigma} \bar{\lambda}_{i}+\lambda_{i} \sigma_{\sigma} \bar{\psi}_{\rho}\right)+\psi_{\mu} \sigma^{\mu \nu} \psi_{\nu} \bar{\lambda}^{i} \bar{\lambda}_{i}+\bar{\psi}_{\mu} \bar{\sigma}^{\mu \nu} \bar{\psi}_{\nu} \lambda^{i} \lambda_{i}
\end{array}
$$

where $\nabla_{\mu}$ is the usual covariant derivative (not the super-covariant one),

$$
\nabla_{\mu}=\partial_{\mu}-A_{\mu}^{i} \delta_{i}-\frac{1}{2} \omega_{\mu}^{a b} l_{a b}
$$

and $F_{\mu \nu}{ }^{i}$ is the supercovariant Yang-Mills field strength,

$$
F_{\mu \nu}^{i}=\partial_{\mu} A_{\nu}^{i}-\partial_{\nu} A_{\mu}^{i}+f_{j k}^{i} A_{\mu}^{j} A_{\nu}^{k}+2 i\left(\lambda^{i} \sigma_{[\mu} \bar{\psi}_{\nu]}+\psi_{[\mu} \sigma_{\nu]} \bar{\lambda}^{i}\right) .
$$

Contributions with chiral matter multiplets and Kähler structure. Kinetic terms for the chiral matter multiplets arise from a contribution to $\Omega$ of the form $K(\varphi, \bar{\varphi})$ with $K$ invariant under all $\delta_{i}$. To see this observe that

$$
\overline{\mathcal{D}}^{2} \mathcal{D}^{2} K(\varphi, \bar{\varphi})=\left(\overline{\mathcal{D}}^{2} \mathcal{D}^{2} \varphi^{s}\right) \frac{\partial K(\varphi, \bar{\varphi})}{\partial \varphi^{s}}+\ldots
$$

where we have introduced an index $s$ labelling the chiral multiplets (instead of interpreting $\varphi$ as a "column vector" in the representation space of $\mathfrak{g}_{\mathrm{YM}}$ as before) and have omitted a bunch of terms. Using (5.41) it is easy to verify that

$$
\overline{\mathcal{D}}^{2} \mathcal{D}^{2} \varphi^{s}=-16 \mathcal{D}_{a} \mathcal{D}^{a} \varphi^{s}+\ldots
$$


where again we omitted many other terms. This shows that a contribution $K(\varphi, \bar{\varphi})$ to $\Omega$ leads to a contribution to the Lagrangian of the form

$$
\begin{aligned}
L_{\text {matter }} & =-16 e \frac{\partial K(\varphi, \bar{\varphi})}{\partial \varphi^{s}} g^{\mu \nu} \partial_{\mu} \partial_{\nu} \varphi^{s}-16 e \frac{\partial K(\varphi, \bar{\varphi})}{\partial \bar{\varphi}^{\bar{s}}} g^{\mu \nu} \partial_{\mu} \partial_{\nu} \bar{\varphi}^{\bar{s}}+\ldots \\
& \sim 32 e \frac{\partial^{2} K(\varphi, \bar{\varphi})}{\partial \varphi^{s} \partial \bar{\varphi}^{\bar{s}}} g^{\mu \nu} \partial_{\mu} \varphi^{s} \partial_{\nu} \bar{\varphi}^{\bar{s}}+\ldots
\end{aligned}
$$

I shall not spell out $L_{\text {matter }}$ in more detail. It has quite a number of terms. I only note that it also involves a term proportional to

$$
e K(\varphi, \bar{\varphi}) \mathcal{R}
$$

which originates from $\overline{\mathcal{D}}^{2} \bar{M} K(\varphi, \bar{\varphi})+$ c.c. owing to (5.37). Hence one actually obtains a Brans-Dicke type action from (5.58) in presence of chiral matter multiplets. To bring this action to the standard (Einstein) form one has to do a redefinition ("Weyl rescaling") of the vierbein according to

$$
\hat{e}_{\mu}^{a} \propto \sqrt{K} e_{\mu}^{a} \Rightarrow e g^{\mu \nu} \propto K^{-1} \hat{e} \hat{g}^{\mu \nu} .
$$

[In order to get a standard form of the action, one usually also redefines similarly the fermion fields.] In terms of the redefined vierbein, (5.65) reads

$$
L_{\text {matter }} \propto \hat{e} G_{s \bar{s}}(\varphi, \bar{\varphi}) \hat{g}^{\mu \nu} \partial_{\mu} \varphi^{s} \partial_{\nu} \bar{\varphi}^{\bar{s}}+\ldots
$$

where we have introduced a Kähler metric in the space of the scalar fields $\varphi^{s}$ and $\bar{\varphi}^{\bar{s}}$ given by

$$
G_{s \bar{s}}(\varphi, \bar{\varphi})=\frac{\partial^{2} \ln K(\varphi, \bar{\varphi})}{\partial \varphi^{s} \partial \bar{\varphi}^{\bar{s}}} \quad \text { (Kähler metric) }
$$

It turns out that the other terms in $L_{\text {matter }}$ can also be expressed nicely in terms of quantities related to the Kähler structure (for instance, there are 4-fermion-terms containing the curvature of $G_{s \bar{s}}$ ). I refer to the textbooks for the details and only add the remark that geometrical structures related to scalar fields are typical of SUGRA theories, also for higher $N$ or $D$. Of course, they are not always Kähler structures as above but of a similar type.

Notice that $L_{\text {matter }}$ can be viewed as a generalization of the pure SUGRA action (5.61) because the latter arises from the special choice $K=$ constant. The YM part (5.62) of the Lagrangian can also be generalized in presence of chiral matter multiplets. Namely a contribution $(-1 / 2) f_{i j}(\bar{\varphi}) \bar{\lambda}^{i} \bar{\lambda}^{j}$ to $P$, with $f_{i j}(\bar{\varphi})$ a symmetric 2-tensor of the YM group, results in a contribution to the Lagrangian of the form

$$
L_{Y M}^{\prime}=e\left[f_{i j}(\bar{\varphi})+\text { c.c. }\right] F_{\mu \nu}^{i} F^{\mu \nu j}+\ldots
$$

This generalizes indeed (5.62) which is just the special case of a constant $f_{i j}$. 
Further invariants. Of course (5.58) can be also used to construct other invariants. In particular, a constant contribution $m$ to $P$ gives rise to

$$
e^{-1} L_{\text {cosmo }}=-3 m M+16 m \psi_{\mu} \sigma^{\mu \nu} \psi_{\nu}+\text { c.c. }
$$

which, when included, contributes to the cosmological constant. Note however that $L_{\text {cosmo }}$ is neither locally nor globally $R$-invariant and is thus forbidden when global or local $R$-invariance is imposed. Furthermore (5.58) can be used to construct higher order invariants containing terms with more than two derivatives. For instance, a contribution of the form $W^{2} \bar{W}^{2} X^{2 n} \bar{X}^{2 n}$ to $\Omega$ results in an invariant containing a contribution $e X^{2(n+1)} \bar{X}^{2(n+1)}$, i.e. a term of order $4(n+1)$ in the Weyl tensor. Such invariants are candidate counterterms in a perturbative quantum field theoretical approach to SUGRA.

\subsection{New minimal SUGRA}

Actually new minimal SUGRA [29] is not fully described by the framework of section 5.1 because it contains a 2 -form gauge potential and is thus a reducible gauge theory. Nevertheless it can be obtained within this framework - it only gives rise to additional formulas for the gauge transformations and Bianchi identities of the 2-form gauge potential and its field strength. The solution to the Bianchi identities is very similar to that of old minimal SUGRA; the differences are that the complex auxiliary field $M$ is zero and the consequences thereof. These consequences arise because $M=0$ requires that the transformations of $M$ must also be zero by consistency. (5.27) and the real part of the right hand side of (5.37) show that this imposes the identifications

$$
M \equiv 0, \quad \lambda_{\alpha}^{(R)} \equiv-\mathrm{i} S_{\alpha}, \quad D^{(R)} \equiv-\frac{1}{4} \mathcal{R}+\frac{3}{2} B_{a} B^{a} .
$$

The imaginary part of the right hand side of (5.37) imposes in addition

$$
\mathcal{D}_{a} B^{a}=0 .
$$

(5.71) shows that in new minimal SUGRA $R$-transformations must be included among the gauge transformations and that $\lambda_{\alpha}^{(R)}$ and $D^{(R)}$ disappear from the list of independent fields. (5.72) must hold as an identity in elementary fields (off-shell). Hence, $B_{a}$ cannot be an independent field either. Rather we must replace it by an expression that satisfies (5.72) identically in the fields and their derivatives. To get an idea how this might work, note that (5.72) is reminiscent of the equation $d \omega_{3}=0$ because of

$$
d \omega_{3}=0, \quad \omega_{3}=\frac{1}{6} d x^{\mu} d x^{\nu} d x^{\rho} f_{\mu \nu \rho} \quad \Leftrightarrow \quad \partial_{\mu} h^{\mu}=0, \quad h^{\mu}=\epsilon^{\mu \nu \rho \sigma} f_{\nu \rho \sigma} .
$$

We know that $d \omega_{3}=0$ is identically solved by

$$
\omega_{3}=d \omega_{2}, \quad \omega_{2}=\frac{1}{2} d x^{\mu} d x^{\nu} f_{\mu \nu} \quad \Leftrightarrow \quad f_{\mu \nu \rho}=3 \partial_{[\mu} f_{\nu \rho]},
$$

where $f_{\nu \rho}$ are arbitrary functions. Notice that $\omega_{2}$ is by no means unique because, owing to $d^{2}=0$, it can be shifted by $d \omega_{1}$ with an arbitrary 1 -form $\omega_{1}$. It turns out that (5.72) can be solved similarly even though it is much more complicated. In particular, it 
contains gravitino dependent terms through the $\omega_{\mu}^{a b}$ occuring in the covariant derivatives $\mathcal{D}_{a}$ and through the terms $E_{a}^{\mu} \psi_{\mu}^{\frac{\alpha}{\mu}} \mathcal{D}_{\underline{\alpha}} B^{a}$ present in $\mathcal{D}_{a} B^{a}$. Notice that the latter terms involve in particular derivatives of the gravitino because, according to (5.29), the transformations $\mathcal{D}_{\underline{\alpha}} B^{a}$ contain the torsions $T_{a b} \underline{\underline{\alpha}}$ which are obtained from (4.25). It is therefore by no means obvious whether or not (5.72) can be satisfied but an explicit computation shows that this is indeed the case. The solution is surprisingly simple:

$$
B^{a} \equiv e^{-1} e_{\mu}^{a} \epsilon^{\mu \nu \rho \sigma}\left(\frac{1}{2} \partial_{\nu} A_{\rho \sigma}+\mathrm{i} \psi_{\nu} \sigma_{\rho} \bar{\psi}_{\sigma}\right)
$$

where $A_{\mu \nu}$ are arbitrary antisymmetric real fields analogous to the $f_{\mu \nu}$ in (5.74). Obviously they are determined only up to redefinitions of the form

$$
A_{\mu \nu}^{\prime}=A_{\mu \nu}+\partial_{\mu} \omega_{\nu}-\partial_{\nu} \omega_{\mu}
$$

for arbitrary $\omega_{\mu}$ (this is completely analogous to the arbitrary shifts $\omega_{2} \rightarrow \omega_{2}+d \omega_{1}$ in the example above). This indicates that $A_{\mu \nu}$ is a 2 -form gauge potential. The gauge transformations are reducible because the gauge parameters $\omega_{\mu}$ can be shifted by $\partial_{\mu} \omega$ with arbitrary $\omega$ without altering (5.76).

Having "solved" (5.72) by (5.75), it is still not clear whether this solution is consistent in the sense that we can assign supersymmetry transformations to $A_{\mu \nu}$ consistently: namely the expression on the right hand side of (5.75) is to transform exactly as $B^{a}$ in old minimal SUGRA with the identifications (5.71) and (5.75). It is not obvious that this is possible because the SUSY transformations of $B_{a}$ in old minimal SUGRA are quite complicated. But, again, this turns out to be the case and the solution is very simple. Together with the diffeomorphism transformations and the gauge transformations (5.76) one obtains the following general gauge transformations of $A_{\mu \nu}$ :

$$
\begin{aligned}
\delta_{\xi, \omega} A_{\mu \nu}= & \partial_{\mu} \omega_{\nu}-\partial_{\nu} \omega_{\mu}+\xi^{\rho} \partial_{\rho} A_{\mu \nu}+\partial_{\mu} \xi^{\rho} A_{\rho \nu}+\partial_{\nu} \xi^{\rho} A_{\mu \rho} \\
& -\mathrm{i}\left(\xi \sigma_{\mu} \bar{\psi}_{\nu}-\xi \sigma_{\nu} \bar{\psi}_{\mu}+\psi_{\mu} \sigma_{\nu} \bar{\xi}-\psi_{\nu} \sigma_{\mu} \bar{\xi}\right) .
\end{aligned}
$$

It follows that the expression on the right hand side of (15.75) is a supercovariant tensor field because in old minimal SUGRA $B^{a}$ is a tensor field. The supercovariant field strength of $A_{\mu \nu}$ can thus be identified with the expression dual to (5.75):

$$
H_{a b c}=E_{a}^{\mu} E_{b}^{\nu} E_{c}^{\rho}\left(3 \partial_{[\mu} A_{\nu \rho]}+6 \mathrm{i} \psi_{[\mu} \sigma_{\nu} \bar{\psi}_{\rho]}\right) .
$$

In terms of $H_{a b c}$, (5.72) reads $\epsilon^{a b c d} \mathcal{D}_{a} H_{b c d}=0$, i.e.,

$$
\mathcal{D}_{[a} H_{b c d]}=0
$$

which can be interpreted as the Bianchi identity for $H_{a b c}$.

The gauge transformations of the other fields are obtained from those given in section 5.3.3 using the identifications (5.71) and (5.75). Together with (5.77) they make up the gauge transformations of new minimal SUGRA with field content

$$
e_{\mu}^{a}, \psi_{\mu}^{\alpha}, A_{\mu \nu}, A_{\mu}^{(R)} ; \quad A_{\mu}^{i}, \lambda_{\alpha}^{i}, D^{i}(i \neq(R)) ; \quad \varphi, \chi_{\alpha}, F .
$$


$\left(e_{\mu}^{a}, \psi_{\mu}^{\alpha}, A_{\mu \nu}, A_{\mu}^{(R)}\right)$ is the new minimal SUGRA multiplet. Notice that it consists solely of gauge fields. Both $A_{\mu \nu}$ and $A_{\mu}^{(R)}$ have three DOF off-shell, and thus the number of bosonic and fermionic DOF match off-shell. As the number of DOF of $e_{\mu}^{a}$ and $\psi_{\mu}$ match on-shell, neither $A_{\mu \nu}$ nor $A_{\mu}^{(R)}$ must have DOF on-shell, i.e., these fields must not propagate (in particular, their DOF on-shell are thus not obtained from (1.1)). This is indeed the case because the pure new minimal SUGRA action reads

$$
\begin{array}{r}
L_{\text {pure }, \text { new }}=\frac{1}{2} e \mathcal{R}+2 \mathrm{i} e\left(S \sigma^{\mu} \bar{\psi}_{\mu}-\psi_{\mu} \sigma^{\mu} \bar{S}\right)+\frac{1}{2} e H_{a b c} H^{a b c}-2 \epsilon^{\mu \nu \rho \sigma} A_{\mu}^{(R)} \partial_{\nu} A_{\rho \sigma} \\
=\frac{1}{2} e R+2 \epsilon^{\mu \nu \rho \sigma}\left(\nabla_{\mu} \psi_{\nu} \sigma_{\rho} \bar{\psi}_{\sigma}+\text { c.c. }\right)+\frac{1}{2} e H_{a b c} H^{a b c}-2 \epsilon^{\mu \nu \rho \sigma} A_{\mu}^{(R)} \partial_{\nu} A_{\rho \sigma}
\end{array}
$$

where $\nabla_{\mu}$ is covariant with respect to Lorentz and $R$-transformations,

$$
\nabla_{\mu} \psi_{\nu}^{\alpha}=\partial_{\mu} \psi_{\nu}^{\alpha}-\frac{1}{2} \omega_{\mu}^{a b}\left(\psi_{\nu} \sigma_{a b}\right)^{\alpha}-\mathrm{i} A_{\mu}^{(R)} \psi_{\nu}^{\alpha}
$$

The EOM for $A_{\mu}^{(R)}$ derived from $L_{\text {pure,new }}$ set $H_{a b c}$ to zero (notice that $A_{\mu}^{(R)}$ occurs in $\nabla_{\mu} \psi_{\nu}$ and $\nabla_{\mu} \bar{\psi}_{\nu}$ ). The EOM for $A_{\mu \nu}$ set the ordinary (non-supercovariant) field strength of $A_{\mu}^{(R)}$ proportional to $\partial_{\rho} H^{\mu \nu \rho}$ and thus, together with the EOM for $A_{\mu}^{(R)}$, this field strength vanishes on-shell. Hence $A_{\mu}^{(R)}$ and $A_{\mu \nu}$ carry indeed no physical DOF.

It was proved in [27] that the most general local function invariant up to a total divergence under the gauge transformations of new minimal SUGRA described above is, up to a total divergence:

$$
\begin{aligned}
L_{\text {new }}= & \mu_{(R)} L_{\text {pure }, \text { new }}+L_{F I}+L_{2} \\
L_{F I}= & \sum_{i_{a}} \mu_{i_{a}}\left(e D^{i_{a}}+e \lambda^{i_{a}} \sigma^{\mu} \bar{\psi}_{\mu}+e \psi_{\mu} \sigma^{\mu} \bar{\lambda}^{i_{a}}+\epsilon^{\mu \nu \rho \sigma} A_{\mu}^{i_{a}} \partial_{\nu} A_{\rho \sigma}\right) \\
L_{2}= & e\left(\overline{\mathcal{D}}^{2}-4 i \psi_{\mu} \sigma^{\mu} \overline{\mathcal{D}}+16 \psi_{\mu} \sigma^{\mu \nu} \psi_{\nu}\right) \mathcal{A}+\text { c.c. }, \\
& \mathcal{A}=P(\bar{W}, \bar{\lambda}, \bar{\varphi})+\mathcal{D}^{2} \Omega(T)
\end{aligned}
$$

where $i_{a}$ are the abelian $i$ different from $(R)$ and the $\mu$ 's are arbitrary constants. $L_{F I}$ is the Fayet-Iliopoulos contribution (redefining the abelian super-YM multiplets by introducing appropriate linear combinations of them, one can achieve that at most one $\mu_{i_{a}}$ is different from zero). Actually $L_{\text {pure,new }}$ is of the same type as the contributions to $L_{F I}$ : in fact it might be viewed as the "Fayet-Iliopoulos contribution" of the $R$-transformation because of (5.71). $\Omega$ and $P$ are again subject to (5.59). The discussion of $L_{2}$ proceeds as the discussion of (5.58) in old minimal SUGRA.

\section{A Lorentz algebra, spinors, Grassmann parity}

\section{A.1 Lorentz algebra}

$D$-dimensional Minkowski metric:

$$
\eta_{a b}=\operatorname{diag}(1,-1, \ldots,-1), \quad a, b \in\{0, \ldots, D-1\} .
$$


Lorentz algebra:

$$
\left[l_{a b}, l_{c d}\right]=\eta_{a d} l_{b c}-\eta_{a c} l_{b d}-(a \leftrightarrow b), \quad l_{a b}=-l_{b a} .
$$

Vector representation of the Lorentz algebra:

$$
l_{a b} V_{c}=\eta_{c b} V_{a}-\eta_{c a} V_{b}, \quad l_{a b} V^{c}=\delta_{b}^{c} V_{a}-\delta_{a}^{c} V_{b}
$$

\section{A.2 Spinor representation in even dimensions}

Dirac algebra $\left(\gamma_{a}\right.$ : complex $2^{D / 2} \times 2^{D / 2}$-matrices $)$ :

$$
\left\{\gamma_{a}, \gamma_{b}\right\}=2 \eta_{a b} \mathbf{1}
$$

The Dirac algebra implies that the matrices

$$
\Sigma_{a b}=\frac{1}{4}\left[\gamma_{a}, \gamma_{b}\right]
$$

form a matrix representation $R$ of the Lorentz algebra (spinor representation):

$$
\left[\Sigma_{a b}, \Sigma_{c d}\right]=\eta_{a d} \Sigma_{b c}-\eta_{a c} \Sigma_{b d}-(a \leftrightarrow b)
$$

Spinors $\Psi$ are complex "column vectors" on which the $\gamma$-matrices act. The Dirac algebra implies that the matrix

$$
\hat{\gamma}=(-\mathrm{i})^{1+D / 2} \gamma_{0} \gamma_{1} \ldots \gamma_{D-1}
$$

satisfies

$$
\hat{\gamma}^{2}=\mathbf{1}, \quad\left\{\hat{\gamma}, \gamma_{a}\right\}=0, \quad\left[\hat{\gamma}, \Sigma_{a b}\right]=0 .
$$

Owing to $\hat{\gamma} \not \subset \mathbf{1}$ and $\left[\hat{\gamma}, \Sigma_{a b}\right]=0, R$ is reducible (Schur's lemma). It decomposes into two inequivalent irreducible representations $R_{+}$and $R_{-}$of the Lorentz algebra, $R=R_{+} \oplus R_{-}$. The corresponding spinors $\Psi_{+}, \Psi_{-}$are called Weyl spinors,

$$
\Psi=\Psi_{+}+\Psi_{-}, \quad \hat{\gamma} \Psi_{ \pm}= \pm \Psi_{ \pm}
$$

Projectors $P_{+}, P_{-}$: owing to $\hat{\gamma}^{2}=\mathbf{1}$, one has

$$
P_{ \pm}=\frac{1}{2}(\mathbf{1} \pm \hat{\gamma}), \quad P_{ \pm}^{2}=P_{ \pm}, \quad P_{+} P_{-}=0=P_{-} P_{+}, \quad P_{+}+P_{-}=1, \quad \Psi_{ \pm}=P_{ \pm} \Psi .
$$

Dirac conjugation, Majorana conjugation, charge conjugation (the terminology used in the literature varies a bit):

$$
\begin{aligned}
& -\kappa \eta \gamma_{a}^{\dagger}=A \gamma_{a} A^{-1}, \quad \bar{\Psi}=\Psi^{\dagger} A \quad \text { (Dirac conjugation); } \\
& \kappa \gamma_{a}^{*}=B^{-1} \gamma_{a} B, \quad \Psi^{c}=B \Psi^{*} \quad \text { (Majorana conjugation); } \\
& -\eta \gamma_{a}^{T}=C^{-1} \gamma_{a} C, \quad \tilde{\Psi}^{c T}=\Psi^{T} C^{-1} \quad \text { (charge conjugation) }
\end{aligned}
$$

where $\kappa, \eta \in\{1,-1\}$. Majorana spinors: $\Psi=B \Psi^{*}$. 


\section{A.3 Spinor representation in odd dimensions}

Can be obtained from a spinor representation in $D=2 k$ by choosing $\gamma_{0}, \ldots, \gamma_{2 k-1}$ as in $D=2 k$ and $\gamma_{2 k}= \pm \mathrm{i} \hat{\gamma}$ with the $\hat{\gamma}$ of the representation in $D=2 k$. There are no Weyl spinors in $D=2 k+1$ (in particular one has $\gamma_{0} \gamma_{1} \ldots \gamma_{2 k} \propto \gamma_{2 k}^{2}=-\mathbf{1}$ ).

For further details see, e.g., [2].

\section{A.4 Spinors in 4 dimensions}

Weyl representation of $\gamma$-matrices:

$$
\begin{aligned}
& \gamma^{a}=\left(\begin{array}{cc}
0 & \sigma^{a} \\
\bar{\sigma}^{a} & 0
\end{array}\right), \quad \gamma_{a}=\eta_{a b} \gamma^{b}, \quad a, b \in\{0,1,2,3\} \\
& \sigma^{0}=\left(\begin{array}{ll}
1 & 0 \\
0 & 1
\end{array}\right), \quad \sigma^{1}=\left(\begin{array}{ll}
0 & 1 \\
1 & 0
\end{array}\right), \quad \sigma^{2}=\left(\begin{array}{rr}
0 & -\mathrm{i} \\
\mathrm{i} & 0
\end{array}\right), \quad \sigma^{3}=\left(\begin{array}{rr}
1 & 0 \\
0 & -1
\end{array}\right), \\
& \bar{\sigma}^{0}=\sigma^{0}, \quad \bar{\sigma}^{1}=-\sigma^{1}, \quad \bar{\sigma}^{2}=-\sigma^{2}, \quad \bar{\sigma}^{3}=-\sigma^{3}
\end{aligned}
$$

Properties:

1. $\hat{\gamma}$ is diagonal: $\hat{\gamma}=\left(\begin{array}{rr}\mathbf{1} & 0 \\ 0 & -\mathbf{1}\end{array}\right) \Rightarrow P_{+}=\left(\begin{array}{ll}\mathbf{1} & 0 \\ 0 & 0\end{array}\right), \quad P_{-}=\left(\begin{array}{ll}0 & 0 \\ 0 & \mathbf{1}\end{array}\right)$ $\Rightarrow$ Weyl spinors reduce to 2-component spinors: $\Psi_{+}=\left(\begin{array}{c}\varphi_{+} \\ 0\end{array}\right), \Psi_{-}=\left(\begin{array}{c}0 \\ \chi_{-}\end{array}\right)$

2. $\quad \Sigma_{a b}=\left(\begin{array}{cc}\sigma_{a b} & 0 \\ 0 & \bar{\sigma}_{a b}\end{array}\right), \quad \sigma_{a b}=\frac{1}{4}\left(\sigma_{a} \bar{\sigma}_{b}-\sigma_{b} \bar{\sigma}_{a}\right), \quad \bar{\sigma}_{a b}=\frac{1}{4}\left(\bar{\sigma}_{a} \sigma_{b}-\bar{\sigma}_{b} \sigma_{a}\right)$

3. all $\gamma$-matrices are unitary: $\gamma_{a}^{-1}=\gamma_{a}^{\dagger}$

4. $\quad A=\gamma^{0}, \quad B=\left(\begin{array}{cc}0 & -\epsilon \\ \epsilon & 0\end{array}\right), \quad C=\left(\begin{array}{rr}-\epsilon & 0 \\ 0 & \epsilon\end{array}\right), \quad \epsilon=\left(\begin{array}{rr}0 & 1 \\ -1 & 0\end{array}\right)$

5. Majorana spinors: $\Psi=\left(\begin{array}{c}\varphi_{+} \\ \epsilon \varphi_{+}^{*}\end{array}\right)$

Infinitesimal Lorentz transformations of $\Psi$ :

$$
l_{a b} \Psi=-\Sigma_{a b} \Psi .
$$

Finite Lorentz transformations with real parameters $\xi^{a b}=-\xi^{b a}$ :

$$
\begin{aligned}
\Psi^{\prime} & =\exp \left(-\frac{1}{2} \xi^{a b} \Sigma_{a b}\right) \Psi=\left(\begin{array}{c}
\Lambda_{+} \varphi_{+} \\
\Lambda_{-} \chi_{-}
\end{array}\right), \\
\Lambda_{+} & =\exp \left(-\frac{1}{2} \xi^{a b} \sigma_{a b}\right) \in \operatorname{SL}(2, \mathbb{C}) \quad\left[\operatorname{SL}(2, \mathbb{C}) \text { because of } \sigma_{a b} \in\left\{ \pm \frac{1}{2} \sigma^{i}, \pm \frac{i}{2} \sigma^{i}\right\}\right], \\
\Lambda_{-} & =\exp \left(-\frac{1}{2} \xi^{a b} \bar{\sigma}_{a b}\right) \stackrel{\bar{\sigma}_{a b}=-\sigma_{a b}^{\dagger}}{=} \exp \left(\frac{1}{2} \xi^{a b} \sigma_{a b}\right)^{\dagger}=\left(\Lambda_{+}\right)^{-1 \dagger} \in \operatorname{SL}(2, \mathbb{C}) .
\end{aligned}
$$

In general: if $D(g)$ is a matrix representation of a group $G$, i.e., $D\left(g_{1}\right) D\left(g_{2}\right)=D\left(g_{1} g_{2}\right)$ for all $g_{1}, g_{2} \in G$, then $[D(g)]^{*},[D(g)]^{-1 T}$ and $[D(g)]^{-1 \dagger}$ are also matrix representations 
of $G$ (owing to $M^{*} N^{*}=(M N)^{*}$ and $M^{-1 T} N^{-1 T}=(M N)^{-1 T}$ for all matrices $M, N$ ). Therefore: in addition to $\Lambda_{+}$and $\Lambda_{-}=\left(\Lambda_{+}\right)^{-1 \dagger}$ one automatically has two further representations of the Lorentz group given by $\left(\Lambda_{+}\right)^{-1 T}$ and $\left(\Lambda_{+}\right)^{*}=\left(\Lambda_{-}\right)^{-1 T}$. However, the latter are equivalent to $\Lambda_{+}$and $\Lambda_{-}=\left(\Lambda_{+}\right)^{-1 \dagger}$, respectively:

$$
\forall M \in \mathrm{SL}(2, \mathbb{C}): \quad M^{-1 T}=\epsilon M \epsilon^{-1}=-\epsilon M \epsilon .
$$

Hence, $\epsilon \varphi_{+}$and $\epsilon \chi_{-}$transform under the Lorentz group according to $\left(\Lambda_{+}\right)^{-1 T}$ and $\left(\Lambda_{+}\right)^{*}$, respectively.

Remark: the last equation is equivalent to $\epsilon=M \epsilon M^{T}$, i.e., $\epsilon$ is $\operatorname{SL}(2, \mathbb{C})$-invariant tensor.

Change of notation: undotted and dotted spinor indices: indices $\alpha \in\{1,2\}$, $\dot{\alpha} \in\{\dot{1}, \dot{2}\}$ indicating the transformation properties under the Lorentz group:

\begin{tabular}{|c|c|l|l|}
\hline new notation & old notation & representation & transformation \\
\hline$\varphi_{\alpha}$ & $\varphi_{+}$ & $\Lambda_{+}$ & $l_{a b} \varphi_{\alpha}=-\left(\sigma_{a b} \varphi\right)_{\alpha}=-\sigma_{a b \alpha}{ }^{\beta} \varphi_{\beta}$ \\
$\varphi^{\alpha}$ & $\epsilon \varphi_{+}$ & $\left(\Lambda_{+}\right)^{-1 T}$ & $l_{a b} \varphi^{\alpha}=\left(\varphi \sigma_{a b}\right)^{\alpha}=\varphi^{\beta} \sigma_{a b \beta}{ }^{\alpha}$ \\
$\bar{\chi}^{\dot{\alpha}}$ & $\chi_{-}$ & $\Lambda_{-}=\left(\Lambda_{+}\right)^{-1 \dagger}$ & $l_{a b} \bar{\chi}^{\dot{\alpha}}=-\left(\bar{\sigma}_{a b} \bar{\chi}\right)^{\dot{\alpha}}=-\bar{\sigma}_{a b} \dot{\alpha} \bar{\chi}^{\dot{\beta}}$ \\
$\bar{\chi}_{\dot{\alpha}}$ & $-\epsilon \chi_{-}$ & $\left(\Lambda_{-}\right)^{-1 T}=\left(\Lambda_{+}\right)^{*}$ & $l_{a b} \bar{\chi}_{\dot{\alpha}}=\left(\bar{\chi} \bar{\sigma}_{a b}\right)_{\dot{\alpha}}=\bar{\chi}_{\dot{\beta}} \bar{\sigma}_{a b} \dot{\alpha} \dot{\alpha}$ \\
\hline
\end{tabular}

Indices of $\sigma$-matrices:

$$
\sigma_{a} \equiv \sigma_{a \alpha \dot{\alpha}}, \quad \bar{\sigma}_{a} \equiv \bar{\sigma}_{a}^{\dot{\alpha} \alpha}, \quad \sigma_{a b} \equiv \sigma_{a b \alpha}{ }^{\beta}, \quad \bar{\sigma}_{a b} \equiv \bar{\sigma}_{a b}{ }_{\dot{\beta}}^{\dot{\alpha}}, \quad \sigma^{a}=\eta^{a b} \sigma_{b} \equiv \sigma_{\alpha \dot{\alpha}}^{a} \quad \text { etc. }
$$

Raising and lowering of spinor indices with $\epsilon$ ("spinor metric"):

$$
\begin{gathered}
\varphi^{\alpha}=\epsilon^{\alpha \beta} \varphi_{\beta}, \quad \varphi_{\alpha}=\epsilon_{\alpha \beta} \varphi^{\beta}, \quad \bar{\chi}_{\dot{\alpha}}=\epsilon_{\dot{\alpha} \dot{\beta}} \bar{\chi}^{\dot{\beta}}, \quad \bar{\chi}^{\dot{\alpha}}=\epsilon^{\dot{\alpha} \dot{\beta}} \bar{\chi}_{\dot{\beta}}, \quad \sigma_{a}{ }_{\dot{\alpha}}=\epsilon^{\alpha \beta} \sigma_{a \beta \dot{\alpha}} \quad \text { etc }, \\
\epsilon^{\alpha \beta}=-\epsilon^{\beta \alpha}, \quad \epsilon_{\alpha \beta}=-\epsilon_{\beta \alpha}, \quad \epsilon^{12}=\epsilon_{21}=1, \\
\epsilon^{\dot{\alpha} \dot{\beta}}=-\epsilon^{\dot{\beta} \dot{\alpha}}, \quad \epsilon_{\dot{\alpha} \dot{\beta}}=-\epsilon_{\dot{\beta} \dot{\alpha}}, \quad \epsilon^{\dot{1} \dot{2}}=\epsilon_{\dot{2} \dot{1}}=1, \\
\Rightarrow \quad \epsilon^{\alpha \gamma} \epsilon_{\gamma \beta}=\delta_{\beta}^{\alpha}, \quad \epsilon^{\dot{\alpha} \dot{\gamma}} \epsilon_{\dot{\gamma} \dot{\beta}}=\delta_{\dot{\beta}}^{\dot{\alpha}} .
\end{gathered}
$$

Complex conjugation:

$$
\left(\psi_{\alpha}\right)^{*}=\bar{\psi}_{\dot{\alpha}}, \quad\left(\psi^{\alpha}\right)^{*}=\bar{\psi}^{\dot{\alpha}}, \quad\left(\bar{\psi}_{\dot{\alpha}}\right)^{*}=\psi_{\alpha}, \quad\left(\bar{\psi}^{\dot{\alpha}}\right)^{*}=\psi^{\alpha}, \quad\left(\psi_{\alpha \beta \dot{\gamma}}\right)^{*}=\bar{\psi}_{\dot{\alpha} \dot{\beta} \gamma} \quad \text { etc. }
$$

Dirac and Majorana spinors:

$$
\text { Dirac spinor: }\left(\begin{array}{c}
\varphi_{\alpha} \\
\bar{\chi}^{\dot{\alpha}}
\end{array}\right), \quad \text { Majorana spinor: }\left(\begin{array}{c}
\psi_{\alpha} \\
\bar{\psi}^{\dot{\alpha}}
\end{array}\right) \text {. }
$$

Notation for contraction of undotted and dotted spinor indices:

$$
\psi \chi \equiv \psi^{\alpha} \chi_{\alpha}, \quad \bar{\psi} \bar{\chi} \equiv \bar{\psi}_{\dot{\alpha}} \bar{\chi}^{\dot{\alpha}}, \quad \sigma_{a} \bar{\sigma}_{b} \equiv\left(\sigma_{a} \bar{\sigma}_{b}\right)_{\alpha}{ }^{\beta} \equiv \sigma_{a \alpha \dot{\alpha}} \bar{\sigma}_{b} \dot{\alpha} \beta \quad \text { etc. }
$$

Vector indices $\rightarrow$ spinor indices:

$$
V_{\alpha \dot{\alpha}}=\sigma_{\alpha \dot{\alpha}}^{a} V_{a}, \quad V^{\dot{\alpha} \alpha}=\bar{\sigma}_{a}^{\dot{\alpha} \alpha} V^{a} .
$$


Remark. Since every vector index can be converted to a pair of spinor indices, vector indices are actually superfluous and so are $\gamma$-matrices and $\sigma$-matrices. In particular, every Lagrangian, EOM, transformation etc can be written in terms of objects carrying only spinor indices, without $\gamma$-matrices or $\sigma$-matrices. When this is done, an expression is only Lorentz invariant if all undotted spinor indices are contracted with $\epsilon_{\alpha \beta}, \epsilon^{\alpha \beta}$ or $\delta_{\alpha}^{\beta}$, and all dotted spinor indices are contracted with $\epsilon_{\dot{\alpha} \dot{\beta}}, \epsilon^{\dot{\alpha} \dot{\beta}}$ or $\delta_{\dot{\alpha}}^{\dot{\beta}}$.

Even though vector indices are superfluous, they are nevertheless still useful, and so are the $\sigma$-matrices (for instance, the use of vector indices may reduce the total number of indices of an object, because one vector index can substitute for two spinor indices). For dealing with the $\sigma$-matrices, the following identities are often useful:

$$
\begin{aligned}
& \bar{\sigma}_{a}^{\dot{\alpha} \alpha}=\sigma_{a}^{\alpha \dot{\alpha}}=\epsilon^{\alpha \beta} \epsilon^{\dot{\alpha} \dot{\beta}} \sigma_{a \beta \dot{\beta}}, \quad \sigma_{a \alpha \dot{\alpha}}=\bar{\sigma}_{a \dot{\alpha} \alpha}=\epsilon_{\alpha \beta} \epsilon_{\dot{\alpha} \dot{\beta}} \bar{\sigma}_{a}^{\dot{\beta} \beta}, \\
& \sigma_{a b}{ }^{\alpha \beta}=\sigma_{a b}{ }^{\beta \alpha}, \quad \sigma_{a b \alpha \beta}=\sigma_{a b \beta \alpha}, \quad \bar{\sigma}_{a b}{ }^{\dot{\alpha} \dot{\beta}}=\bar{\sigma}_{a b} \dot{\beta} \dot{\alpha}, \quad \bar{\sigma}_{a b \dot{\alpha} \dot{\beta}}=\bar{\sigma}_{a b \dot{\beta} \dot{\alpha}}, \\
& \left(\sigma^{a} \bar{\sigma}^{b}\right)_{\alpha}^{\beta}=\eta^{a b} \delta_{\alpha}^{\beta}+2 \sigma^{a b}{ }_{\alpha}^{\beta}, \quad\left(\bar{\sigma}^{a} \sigma^{b}\right)_{\dot{\beta}}^{\dot{\alpha}}=\eta^{a b} \delta_{\dot{\beta}}^{\dot{\alpha}}+2 \bar{\sigma}_{\dot{\beta}}^{a b \dot{\alpha}}, \\
& \sigma_{a \alpha \dot{\alpha}} \sigma_{\beta \dot{\beta}}^{a}=2 \epsilon_{\alpha \beta} \epsilon_{\dot{\alpha} \dot{\beta}}, \quad \bar{\sigma}^{a \dot{\alpha} \alpha} \bar{\sigma}_{a}^{\dot{\beta} \beta}=2 \epsilon^{\dot{\alpha} \dot{\beta}} \epsilon^{\alpha \beta}, \quad \sigma^{a}{ }_{\alpha \dot{\alpha}} \bar{\sigma}_{a}{ }^{\beta \dot{\beta}}=2 \delta_{\alpha}^{\beta} \delta_{\dot{\alpha}}^{\dot{\beta}}, \\
& \epsilon^{a b c d} \sigma_{c d}=2 \mathrm{i} \sigma^{a b}, \quad \epsilon^{a b c d} \bar{\sigma}_{c d}=-2 \mathrm{i} \bar{\sigma}^{a b}, \quad \epsilon^{0123}=1, \\
& \sigma^{a b} \sigma^{c}=\frac{1}{2}\left(\eta^{b c} \sigma^{a}-\eta^{a c} \sigma^{b}+\mathrm{i} \epsilon^{a b c d} \sigma_{d}\right), \\
& \sigma^{c} \bar{\sigma}^{a b}=\frac{1}{2}\left(-\eta^{b c} \sigma^{a}+\eta^{a c} \sigma^{b}+\mathrm{i} \epsilon^{a b c d} \sigma_{d}\right), \\
& \bar{\sigma}^{a b} \bar{\sigma}^{c}=\frac{1}{2}\left(\eta^{b c} \bar{\sigma}^{a}-\eta^{a c} \bar{\sigma}^{b}-\mathrm{i} \epsilon^{a b c d} \bar{\sigma}_{d}\right), \\
& \bar{\sigma}^{c} \sigma^{a b}=\frac{1}{2}\left(-\eta^{b c} \bar{\sigma}^{a}+\eta^{a c} \bar{\sigma}^{b}-\mathrm{i} \epsilon^{a b c d} \bar{\sigma}_{d}\right) .
\end{aligned}
$$

\section{A.5 Grassmann parity}

Generalization of wedge product for differential forms:

$$
X Y=(-)^{|X||Y|} Y X, \quad\left|T_{\alpha_{1} \ldots \alpha_{n}}^{\dot{\alpha}_{1} \ldots \dot{\alpha}_{m}}\right|=(m+n+\text { form-degree }) \bmod 2,
$$

where $X, Y, T$ are fields or differential forms. $|X|$ is called the Grassmann parity (or simply the parity) of $X 7$

Complex conjugation of products:

$$
(X Y)^{*}=(-)^{|X||Y|} X^{*} Y^{*}
$$

Simple consequences:

$$
\begin{aligned}
& \psi \chi=\psi^{\alpha} \chi_{\alpha}=\epsilon^{\alpha \beta} \psi_{\beta} \chi_{\alpha}=-\epsilon^{\alpha \beta} \chi_{\alpha} \psi_{\beta}=\epsilon^{\beta \alpha} \chi_{\alpha} \psi_{\beta}=\chi \psi, \\
& (\psi \chi)^{*}=\left(\psi^{\alpha} \chi_{\alpha}\right)^{*}=-\bar{\psi}^{\dot{\alpha}} \bar{\chi}_{\dot{\alpha}}=+\bar{\chi}_{\dot{\alpha}} \bar{\psi}^{\dot{\alpha}}=\bar{\chi} \bar{\psi} .
\end{aligned}
$$

\footnotetext{
${ }^{7}$ In the BRST approach the definition of the Grassmann parity involves the ghost number in addition to the number of spinor indices and the form-degree.
} 


\section{B Explicit verification of local SUSY}

\section{B.1 Local SUSY of (3.3)}

1.5 order formalism. This is a "trick" to simplify the variation of a second order action if it derives from a first order one. The argument is simple and general: suppose a Lagrangian $L(\phi, H)$ involves fields $\phi^{i}$ and $H^{A}$ such that the EOM for the $H^{A}$ have the algebraic solution $H^{A}=H^{A}(\phi) 8$ Let us now consider the second order Lagrangian $L(\phi, H(\phi))$ and vary the fields $\phi^{i}$. We obtain

$$
\delta L(\phi, H(\phi)) \sim\left[\delta \phi^{i} \frac{\hat{\partial} L(\phi, H)}{\hat{\partial} \phi^{i}}+\delta H^{A}(\phi) \frac{\hat{\partial} L(\phi, H)}{\hat{\partial} H^{A}}\right]_{H=H(\phi)}=\left[\delta \phi^{i} \frac{\hat{\partial} L(\phi, H)}{\hat{\partial} \phi^{i}}\right]_{H=H(\phi)} .
$$

Here $L(\phi, H)$ is the first order Lagrangian, $\sim$ denotes equality up to a total divergence, and $\delta H^{A}(\phi)=H^{A}(\phi+\delta \phi)-H^{A}(\phi)$ is the variation of $H^{A}(\phi)$. The terms with $\delta H^{A}(\phi)$ on the right hand side vanish (no matter what the $\delta H^{A}(\phi)$ are) because the $H^{A}(\phi)$ algebraically solve the EOM of the $H$ 's which means

$$
\left.\frac{\hat{\partial} L(\phi, H)}{\hat{\partial} H^{A}}\right|_{H=H(\phi)}=0 \quad \text { (identically). }
$$

We observe that, up to a total derivative, the variation of the second order Lagrangian $L(\phi, H(\phi))$ is obtained from varying only the $\phi^{i}$ (but not the $H^{A}$ ) in the first order Lagrangian $L(\phi, H)$ and substituting $H^{A}(\phi)$ for $H^{A}$ afterwards. Hence, one uses the first order action to compute the variation of the second order one. This motivates the term "1.5 order formalism". Notice that the argument applies to all variations $\delta$. In particular it shows that the EOM of the second order formulation can be obtained from those of the first order formulation according to

$$
\frac{\hat{\partial} L(\phi, H(\phi))}{\hat{\partial} \phi^{i}}=\left.\frac{\hat{\partial} L(\phi, H)}{\hat{\partial} \phi^{i}}\right|_{H=H(\phi)} .
$$

Furthermore it can be used to verify invariance of the second order Lagrangian under symmetry transformations.

Verification of SUSY. Using the 1.5 order formalism, we shall now demonstrate the SUSY of the Lagrangian (3.3) in the second order formulation under the SUSY transformations (3.14) through (3.16). The advantage of the 1.5 order formalism is that we do not need to transform the spin connection $\omega$ but only the vierbein and gravitino, using the first order Lagrangian. In fact, we can further simplify the calculation by using only the part $\delta_{+}$of the SUSY transformations of the vierbein and gravitino which involve the SUSY parameters $\xi^{\alpha}$ but not their complex conjugates $\bar{\xi}^{\dot{\alpha}}$ :

$$
\delta_{+} e_{\mu}^{a}=2 \mathrm{i} \xi \sigma^{a} \bar{\psi}_{\mu}, \quad \delta_{+} \psi_{\mu}^{\alpha}=\nabla_{\mu} \xi^{\alpha}, \quad \delta_{+} \bar{\psi}_{\mu}^{\dot{\alpha}}=0, \quad \delta_{+} \omega_{\mu}^{a b}=0 .
$$

\footnotetext{
${ }^{8}$ To simplify formulae, we use here the notation $L(\phi, H)$ and $H(\phi)$ in place of $L([\phi, H])$ and $H([\phi])$.
} 
The reason is that the other part $\delta_{-}$, involving the $\bar{\xi}^{\dot{\alpha}}$, is the complex conjugate of $\delta_{+}$, and thus, since the Lagrangian is real:

$$
\delta_{-} L=\left(\delta_{+} L\right)^{*} .
$$

Hence $\left[\delta_{+} L(e, \psi, \omega)\right]_{\omega=\omega(e, \psi)}=\partial_{\mu} K_{+}^{\mu}$ implies $\left[\delta_{-} L(e, \psi, \omega)\right]_{\omega=\omega(e, \psi)}=\partial_{\mu} K_{-}^{\mu}$ with $K_{-}^{\mu}=$ $\left(K_{+}^{\mu}\right)^{*}$. Conversely, $\left[\delta_{\text {susy }} L(e, \psi, \omega)\right]_{\omega=\omega(e, \psi)}=\partial_{\mu} K^{\mu}$ requires that $\left[\delta_{+} L(e, \psi, \omega)\right]_{\omega=\omega(e, \psi)}$ be a total divergence [remember that local SUSY requires invariance up to a total divergence for arbitrary complex parameters, i.e., we may consider $\xi$ and $\bar{\xi}$ as independent fields (instead of their real and imaginary parts)]. Hence $\left[\delta_{+} L(e, \psi, \omega)\right]_{\omega=\omega(e, \psi)} \sim 0$ is necessary and sufficient for $\delta_{\text {susy }} L \sim 0$ (again, " $\sim$ " denotes equality up to a total divergence).

Transformation of the "Einstein-part":

$$
\begin{aligned}
\frac{1}{2} \delta_{+}\left[e E_{b}^{\mu} E_{a}^{\nu} R_{\mu \nu}^{a b}(\omega)\right] & =\frac{1}{2} \underbrace{\left(\delta_{+} e\right)}_{e E_{c}^{\rho} \delta_{+} e_{\rho}^{c}} E_{b}^{\mu} E_{a}^{\nu} R_{\mu \nu}{ }^{a b}(\omega)+e \underbrace{\left(\delta_{+} E_{b}^{\mu}\right)}_{-E_{b}^{\rho} E_{c}^{\mu} \delta_{+} e_{\rho}^{c}} E_{a}^{\nu} R_{\mu \nu}{ }^{a b}(\omega) \\
& =e\left(\delta_{+} e_{\rho}^{c}\right)\left(\frac{1}{2} E_{c}^{\rho} R-R_{c}{ }^{\rho}\right)=\underbrace{\mathrm{i} e\left(\xi \sigma^{\mu} \bar{\psi}_{\mu} R-2 \xi \sigma^{a} \bar{\psi}_{\mu} R_{a}{ }^{\mu}\right)}_{1},
\end{aligned}
$$

where $R_{a}{ }^{\mu}=R_{\rho \nu}{ }^{b c} E_{a}^{\rho} E_{b}^{\nu} E_{c}^{\mu}$. Transformation of the "gravitino-part":

$$
\begin{aligned}
2 \epsilon^{\mu \nu \rho \sigma} \delta_{+}\left(\nabla_{\mu} \psi_{\nu} \sigma_{\rho} \bar{\psi}_{\sigma}+\psi_{\sigma} \sigma_{\rho} \nabla_{\mu} \bar{\psi}_{\nu}\right) & =\underbrace{2 \epsilon^{\mu \nu \rho \sigma}\left(\nabla_{\mu} \delta_{+} \psi_{\nu}\right) \sigma_{\rho} \bar{\psi}_{\sigma}}_{2}+\underbrace{2 \epsilon^{\mu \nu \rho \sigma} \nabla_{\mu} \psi_{\nu}\left(\delta_{+} \sigma_{\rho}\right) \bar{\psi}_{\sigma}}_{3} \\
& +\underbrace{2 \epsilon^{\mu \nu \rho \sigma}\left(\delta_{+} \psi_{\sigma}\right) \sigma_{\rho} \nabla_{\mu} \bar{\psi}_{\nu}}_{4}+\underbrace{2 \epsilon^{\mu \nu \rho \sigma} \psi_{\sigma}\left(\delta_{+} \sigma_{\rho}\right) \nabla_{\mu} \bar{\psi}_{\nu}}_{5} .
\end{aligned}
$$

Individual terms: $\nabla_{[\mu} \delta_{+} \psi_{\nu]}=\nabla_{[\mu} \nabla_{\nu]} \xi=\frac{1}{2}\left[\nabla_{\mu}, \nabla_{\nu}\right] \xi=-\frac{1}{4} R_{\mu \nu}^{a b}(\omega) l_{a b} \xi \Rightarrow$

$$
\begin{gathered}
2=-\frac{1}{2} \epsilon^{\mu \nu \rho \sigma} R_{\mu \nu}^{a b}(\omega) \xi \sigma_{a b} \sigma_{\rho} \bar{\psi}_{\sigma} \\
\delta_{+} \sigma_{\rho \alpha \dot{\alpha}}=\sigma_{a \alpha \dot{\alpha}} \delta_{+} e_{\rho}^{a}=2 \mathrm{i} \sigma_{a \alpha \dot{\alpha}} \xi \sigma^{a} \bar{\psi}_{\rho}=4 \mathrm{i} \xi_{\alpha} \bar{\psi}_{\rho \dot{\alpha}} \Rightarrow \\
3=8 \mathrm{i} \epsilon^{\mu \nu \rho \sigma} \xi \nabla_{\mu} \psi_{\nu} \underbrace{\bar{\psi}_{\rho} \bar{\psi}_{\sigma}}_{=\bar{\psi}_{(\sigma} \bar{\psi}_{\rho)}}=0, \quad 5=8 \mathrm{i} \epsilon^{\mu \nu \rho \sigma} \xi \psi_{\sigma} \bar{\psi}_{\rho} \nabla_{\mu} \bar{\psi}_{\nu} .
\end{gathered}
$$

Fourth term: "integration by parts" to remove derivatives from $\xi$ :

$$
\begin{aligned}
4 & =\underbrace{\nabla_{\sigma}\left(2 \epsilon^{\mu \nu \rho \sigma} \xi \sigma_{\rho} \nabla_{\mu} \bar{\psi}_{\nu}\right)}_{\partial_{\sigma}\left(2 \epsilon^{\mu \nu \rho \sigma} \xi \sigma_{\rho} \nabla_{\mu} \bar{\psi}_{\nu}\right)} \underbrace{-2 \epsilon^{\mu \nu \rho \sigma} \xi\left(\nabla_{\sigma} \sigma_{\rho}\right) \nabla_{\mu} \bar{\psi}_{\nu}}_{4 \mathrm{a}} \underbrace{-2 \epsilon^{2}}_{4 \epsilon^{\mu \nu \rho \sigma} \xi \sigma_{\rho} \nabla_{\sigma} \nabla_{\mu} \bar{\psi}_{\nu}}, \\
\left.4 \mathrm{a}\right|_{\omega=\omega(e, \psi)} & =-\left.2 \epsilon^{\mu \nu \rho \sigma}\left(\left.\nabla\right|_{[\sigma} e_{\rho]}^{a}\right) \xi \sigma_{a} \nabla\right|_{\mu} \bar{\psi}_{\nu} \stackrel{3.4}{=}-\left.4 \mathrm{i} \epsilon^{\mu \nu \rho \sigma}\left(\psi_{\sigma} \sigma^{a} \bar{\psi}_{\rho}\right) \xi \sigma_{a} \nabla\right|_{\mu} \bar{\psi}_{\nu} \\
& =-\left.8 \mathrm{i} \epsilon^{\mu \nu \rho \sigma} \xi \psi_{\sigma} \bar{\psi}_{\rho} \nabla\right|_{\mu} \bar{\psi}_{\nu} \quad \text { where }\left.\nabla\right|_{\mu}=\partial_{\mu}-\frac{1}{2} \omega_{\mu}^{a b}(e, \psi) l_{a b}, \\
4 \mathrm{~b} & =-2 \epsilon^{\mu \nu \rho \sigma} \xi \sigma_{\rho} \frac{1}{2}\left[\nabla_{\sigma}, \nabla_{\mu}\right] \bar{\psi}_{\nu}=-\frac{1}{2} \epsilon^{\mu \nu \rho \sigma} \xi \sigma_{\rho} R_{\sigma \mu}{ }^{a b}(\omega) \bar{\sigma}_{a b} \bar{\psi}_{\nu} .
\end{aligned}
$$


Terms 1, 2 and 4b cancel out [computation is similar to a computation before (3.4)]:

$$
\begin{gathered}
2+4 \mathrm{~b}=-\frac{1}{2} \epsilon^{\mu \nu \rho \sigma} R_{\mu \nu}^{a b}(\omega) \xi \underbrace{\left(\sigma_{a b} \sigma_{\rho}+\sigma_{\rho} \bar{\sigma}_{a b}\right)}_{=\mathrm{i} \epsilon_{a b \rho c} \sigma^{c}} \bar{\psi}_{\sigma}=\ldots=-1 \\
\Rightarrow\left[\delta_{+} L(e, \psi, \omega)\right]_{\omega=\omega(e, \psi)} \sim[\underbrace{11+2+4 \mathrm{~b}}_{=0}+\underbrace{[3}_{=0}+\underbrace{\sqrt{4 \mathrm{a}}+\underbrace{5}_{\omega=\omega(e, \psi)}}_{=0}=0, \quad \text { qed. }
\end{gathered}
$$

\section{B.2 Local SUSY of (5.58)}

Let us verify explicitly the invariance of (5.58) up to a total divergence under the local SUSY-transformations given in section 5.3 .3 (using unhatted parameters). Let us start with the terms coming from the transformation of $e$ which is given by

$$
\delta_{\text {susy }} e=e E_{a}^{\mu} \delta_{\text {susy }} e_{\mu}^{a}=e E_{a}^{\mu}\left(2 \mathrm{i} \xi \sigma^{a} \bar{\psi}_{\mu}-2 \mathrm{i} \psi_{\mu} \sigma^{a} \bar{\xi}\right)=2 \mathrm{i} e\left(\xi \sigma^{\mu} \bar{\psi}_{\mu}-\psi_{\mu} \sigma^{\mu} \bar{\xi}\right)
$$

This gives:

$$
\begin{aligned}
& \left(\delta_{\text {susy }} e\right)\left(\overline{\mathcal{D}}^{2}-4 \mathrm{i} \psi_{\mu} \sigma^{\mu} \overline{\mathcal{D}}-3 M+16 \psi_{\mu} \sigma^{\mu \nu} \psi_{\nu}\right) \mathcal{A} \\
& \quad=2 \mathrm{i} e\left(\xi \sigma^{\rho} \bar{\psi}_{\rho}-\psi_{\rho} \sigma^{\rho} \bar{\xi}\right)\left(\overline{\mathcal{D}}^{2}-4 \mathrm{i} \psi_{\mu} \sigma^{\mu} \overline{\mathcal{D}}-3 M+16 \psi_{\mu} \sigma^{\mu \nu} \psi_{\nu}\right) \mathcal{A}
\end{aligned}
$$

To evaluate the other contributions we shall use that $\mathcal{A}$ by construction is antichiral:

$$
\mathcal{D}_{\alpha} \mathcal{A}=0 .
$$

This holds because $P$ is antichiral, as it is a function of antichiral tensor fields,

$$
\mathcal{D}_{\alpha} \bar{W}_{\dot{\alpha} \dot{\beta} \dot{\gamma}}=0, \quad \mathcal{D}_{\alpha} \bar{\lambda}_{\dot{\alpha}}^{i}=0, \quad \mathcal{D}_{\alpha} \bar{\varphi}=0,
$$

and because $\left(\mathcal{D}^{2}-\bar{M}\right) \Omega(T)$ is also antichiral, since $\left(\mathcal{D}^{2}-\bar{M}\right) f(T)$ is antichiral for every $l_{\alpha \beta}$-invariant function $f(T)$ :

$$
l_{\alpha \beta} f(T)=0 \quad \Rightarrow \quad \mathcal{D}_{\alpha}\left(\mathcal{D}^{2}-\bar{M}\right) f(T)=0
$$

(B.5) can be deduced from the calculation of $\mathcal{D}_{\alpha} F$ in section 5.3.2, see (5.43) and the equations subsequent to it: namely, the result of that calculation was $\mathcal{D}_{\alpha} F=-\frac{1}{2} \bar{M} \chi_{\alpha}$ which can also be written as $-\frac{1}{2} \mathcal{D}_{\alpha} \mathcal{D}^{2} \varphi=-\frac{1}{2} \bar{M} \mathcal{D}_{\alpha} \varphi$ or, equivalently, as $\mathcal{D}_{\alpha}\left(\mathcal{D}^{2}-M\right) \varphi=$ 0 . As one can check, the derivation given in section 5.3 .2 made only use of the (anti)commutators $\left[\mathcal{D}_{\alpha}, \mathcal{D}_{\beta}\right]=\bar{M} l_{\alpha \beta}$ and of $l_{\alpha \beta} \varphi=0$. Hence, it actually goes also through with $\varphi$ replaced by any $l_{\alpha \beta^{-}}$invariant function of tensor fields, which yields (B.5).

Let us now consider $e \delta_{\text {susy }} \overline{\mathcal{D}}^{2} \mathcal{A}$. Since $\overline{\mathcal{D}}^{2} \mathcal{A}$ is a (composite) tensor field, we have

$$
e \delta_{\text {susy }} \overline{\mathcal{D}}^{2} \mathcal{A}=e\left(\xi^{\alpha} \mathcal{D}_{\alpha}+\bar{\xi}_{\dot{\alpha}} \overline{\mathcal{D}}^{\dot{\alpha}}\right) \overline{\mathcal{D}}^{2} \mathcal{A}
$$

By the complex conjugate of (B.5) the second term on the right hand side is

$$
e \bar{\xi}_{\dot{\alpha}} \overline{\mathcal{D}}^{\dot{\alpha}} \overline{\mathcal{D}}^{2} \mathcal{A}=e M \bar{\xi}_{\dot{\alpha}} \overline{\mathcal{D}}^{\dot{\alpha}} \mathcal{A}
$$


The evaluation of $e \xi^{\alpha} \mathcal{D}_{\alpha} \overline{\mathcal{D}}^{2} \mathcal{A}$ requires more work. We treat it as follows: we use the graded commutator algebra (5.24) to pass $\mathcal{D}_{\alpha}$ through $\overline{\mathcal{D}}^{2}$ until it hits $\mathcal{A}$ where it produces a 0 because of (B.4). Furthermore we bring the covariant derivatives which arise to the left of the spinor transformations, using again the graded commutator algebra:

$$
\begin{aligned}
e \xi^{\alpha} \mathcal{D}_{\alpha} \overline{\mathcal{D}}^{2} \mathcal{A} & =e \xi^{\alpha}\left(\left[\mathcal{D}_{\alpha}, \overline{\mathcal{D}}_{\dot{\alpha}}\right] \overline{\mathcal{D}}^{\dot{\alpha}}-\overline{\mathcal{D}}_{\dot{\alpha}}\left[\mathcal{D}_{\alpha}, \overline{\mathcal{D}}^{\dot{\alpha}}\right]\right) \mathcal{A} \\
& =e \xi^{\alpha}\left(-2 \mathrm{i} \mathcal{D}_{\alpha \dot{\alpha}} \overline{\mathcal{D}}^{\dot{\alpha}}-2 B_{\alpha \dot{\beta}} \bar{l}_{\dot{\alpha}}^{\dot{\beta}} \overline{\mathcal{D}}^{\dot{\alpha}}-2 \mathrm{i} \overline{\mathcal{D}}^{\dot{\alpha}} \mathcal{D}_{\alpha \dot{\alpha}}\right) \mathcal{A} \\
& =e \xi^{\alpha}\left(-2 \mathrm{i} \mathcal{D}_{\alpha \dot{\alpha}} \overline{\mathcal{D}}^{\dot{\alpha}}+3 B_{\alpha \dot{\alpha}} \overline{\mathcal{D}}^{\dot{\alpha}}-2 \mathrm{i}\left[\overline{\mathcal{D}}^{\dot{\alpha}}, \mathcal{D}_{\alpha \dot{\alpha}}\right]-2 \mathrm{i} \mathcal{D}_{\alpha \dot{\alpha}} \overline{\mathcal{D}}^{\dot{\alpha}}\right) \mathcal{A} \\
& =e \xi^{\alpha}\left(-2 \mathrm{i} \mathcal{D}_{\alpha \dot{\alpha}} \overline{\mathcal{D}}^{\dot{\alpha}}+3 B_{\alpha \dot{\alpha}} \overline{\mathcal{D}}^{\dot{\alpha}}+8 \lambda_{\alpha}^{(R)} \delta_{(R)}-5 B_{\alpha \dot{\alpha}} \overline{\mathcal{D}}^{\dot{\alpha}}-2 \mathrm{i} \mathcal{D}_{\alpha \dot{\alpha}} \overline{\mathcal{D}}^{\dot{\alpha}}\right) \mathcal{A} \\
& =e \xi^{\alpha}\left(-4 \mathrm{i} \mathcal{D}_{\alpha \dot{\alpha}} \overline{\mathcal{D}}^{\dot{\alpha}}-2 B_{\alpha \dot{\alpha}} \overline{\mathcal{D}}^{\dot{\alpha}}-16 \mathrm{i} \lambda_{\alpha}^{(R)}\right) \mathcal{A},
\end{aligned}
$$

where we used (B.4) and (5.59). Finally we evaluate analogously the gravitino dependent terms of the supercovariant derivative in the last line:

$$
\begin{aligned}
\mathcal{D}_{\alpha \dot{\alpha}} \overline{\mathcal{D}}^{\dot{\alpha}} \mathcal{A} & =\sigma_{\alpha \dot{\alpha}}^{\mu}\left(\nabla_{\mu}-\psi_{\mu}^{\beta} \mathcal{D}_{\beta}-\bar{\psi}_{\mu \dot{\beta}} \overline{\mathcal{D}}^{\dot{\beta}}\right) \overline{\mathcal{D}}^{\dot{\alpha}} \mathcal{A} \\
& =\sigma_{\alpha \dot{\alpha}}^{\mu}\left(\nabla_{\mu} \overline{\mathcal{D}}^{\dot{\alpha}}-\psi_{\mu}^{\beta}\left[\mathcal{D}_{\beta}, \overline{\mathcal{D}}^{\dot{\alpha}}\right]+\frac{1}{2} \bar{\psi}_{\mu}^{\dot{\alpha}} \overline{\mathcal{D}}^{2}\right) \mathcal{A} \\
& =\sigma_{\alpha \dot{\alpha}}^{\mu}\left(\nabla_{\mu} \overline{\mathcal{D}}^{\dot{\alpha}}-2 \mathrm{i} \psi_{\mu \beta} \bar{\sigma}^{\nu \dot{\alpha} \beta}\left(\nabla_{\nu}-\bar{\psi}_{\nu} \overline{\mathcal{D}}\right)+\frac{1}{2} \bar{\psi}_{\mu}^{\dot{\alpha}} \overline{\mathcal{D}}^{2}\right) \mathcal{A} \\
& =\left(\sigma_{\alpha \dot{\alpha}}^{\mu} \nabla_{\mu} \overline{\mathcal{D}}^{\dot{\alpha}}-2 \mathrm{i}\left(\sigma^{\mu} \bar{\sigma}^{\nu} \psi_{\mu}\right)_{\alpha}\left(\nabla_{\nu}-\bar{\psi}_{\nu} \overline{\mathcal{D}}\right)+\frac{1}{2}\left(\sigma^{\mu} \bar{\psi}_{\mu}\right)_{\alpha} \overline{\mathcal{D}}^{2}\right) \mathcal{A}
\end{aligned}
$$

where $\nabla_{\mu}$ is covariant with regard to Lorentz and $R$-transformations. Collecting all terms we obtain

$$
\begin{aligned}
e \delta_{\text {susy }} \overline{\mathcal{D}}^{2} \mathcal{A}= & e\left(-4 \mathrm{i} \xi \sigma^{\mu} \nabla_{\mu} \overline{\mathcal{D}}-8 \xi \sigma^{\mu} \bar{\sigma}^{\nu} \psi_{\mu} \nabla_{\nu}+8 \xi \sigma^{\mu} \bar{\sigma}^{\nu} \psi_{\mu} \bar{\psi}_{\nu} \overline{\mathcal{D}}\right. \\
& \left.-2 \mathrm{i} \xi \sigma^{\mu} \bar{\psi}_{\mu} \overline{\mathcal{D}}^{2}-2 B_{a} \xi \sigma^{a} \overline{\mathcal{D}}-16 \mathrm{i} \xi \lambda^{(R)}+M \bar{\xi} \overline{\mathcal{D}}\right) \mathcal{A}
\end{aligned}
$$

Next we compute the SUSY transformation of $-4 \mathrm{i} e \delta_{\text {susy }}\left(\psi_{\mu} \sigma^{\mu} \overline{\mathcal{D}} \mathcal{A}\right)$. We obtain, using $\delta_{\text {susy }} E_{a}^{\mu}=-E_{b}^{\mu} E_{a}^{\nu} \delta_{\text {susy }} e_{\nu}^{b}$ and manipulations as above:

$$
\begin{aligned}
-4 \mathrm{i} e & \delta_{\text {susy }}\left(\psi_{\mu} \sigma^{\mu} \overline{\mathcal{D}} \mathcal{A}\right) \\
= & -4 \mathrm{i} e\left[\left(\delta_{\text {susy }} \psi_{\mu}\right) \sigma^{\mu} \overline{\mathcal{D}}+\left(\delta_{\text {susy }} E_{a}^{\mu}\right) \psi_{\mu} \sigma^{a} \overline{\mathcal{D}}+\psi_{\mu}^{\alpha} \sigma_{\alpha \dot{\alpha}}^{\mu}\left(\xi^{\beta} \mathcal{D}_{\beta}+\bar{\xi}_{\dot{\beta}} \overline{\mathcal{D}}^{\dot{\beta}}\right) \overline{\mathcal{D}}^{\dot{\alpha}}\right] \mathcal{A} \\
= & -4 \mathrm{i} e\left(\nabla_{\mu} \xi-\mathrm{i} B_{\mu} \xi+\mathrm{i} B^{\nu} \xi \sigma_{\nu \mu}+\frac{\mathrm{i}}{8} M \bar{\xi} \bar{\sigma}_{\mu}\right) \sigma^{\mu} \overline{\mathcal{D}} \mathcal{A} \\
& +4 \mathrm{i} e E_{b}^{\mu} E_{a}^{\nu}\left(2 \mathrm{i} \xi \sigma^{b} \bar{\psi}_{\nu}-2 \mathrm{i} \psi_{\nu} \sigma^{b} \bar{\xi}\right) \psi_{\mu} \sigma^{a} \overline{\mathcal{D}} \mathcal{A} \\
& +8 e \psi_{\mu} \sigma^{\mu} \bar{\sigma}^{\nu} \xi\left(\nabla_{\nu}-\bar{\psi}_{\nu} \overline{\mathcal{D}}\right) \mathcal{A}+2 \mathrm{i} e \psi_{\mu} \sigma^{\mu} \bar{\xi} \overline{\mathcal{D}}^{2} \mathcal{A} \\
= & -4 \mathrm{i} e \nabla_{\mu} \xi \sigma^{\mu} \overline{\mathcal{D}} \mathcal{A}+2 e B_{\mu} \xi \sigma^{\mu} \overline{\mathcal{D}} \mathcal{A}+2 e M \bar{\xi} \overline{\mathcal{D}} \mathcal{A} \\
& +8 e\left(\psi_{\nu} \sigma^{\mu} \bar{\xi}-\xi \sigma^{\mu} \bar{\psi}_{\nu}\right) \psi_{\mu} \sigma^{\nu} \overline{\mathcal{D}} \mathcal{A} \\
& +8 e \psi_{\mu} \sigma^{\mu} \bar{\sigma}^{\nu} \xi\left(\nabla_{\nu}-\bar{\psi}_{\nu} \overline{\mathcal{D}}\right) \mathcal{A}+2 \mathrm{i} e \psi_{\mu} \sigma^{\mu} \bar{\xi} \overline{\mathcal{D}}^{2} \mathcal{A} .
\end{aligned}
$$

To compute $-3 e \delta_{\text {susy }}(M \mathcal{A})$ we use that (4.25) gives explicitly:

$$
S_{\alpha}=-\left(\sigma^{a b} T_{a b}\right)_{\alpha}=\left(-2 \sigma^{\mu \nu} \nabla_{\mu} \psi_{\nu}+\frac{3 \mathrm{i}}{2} B^{\mu} \psi_{\mu}-\frac{3 \mathrm{i}}{8} M \sigma^{\mu} \bar{\psi}_{\mu}\right)_{\alpha} .
$$

This yields:

$$
\begin{aligned}
& -3 e \delta_{\text {susy }}(M \mathcal{A})=-16 e\left(\xi S-\mathrm{i} \xi \lambda^{(R)}\right) \mathcal{A}-3 e M \bar{\xi} \overline{\mathcal{D}} \mathcal{A} \\
& \quad=e\left(32 \xi \sigma^{\mu \nu} \nabla_{\mu} \psi_{\nu}-24 \mathrm{i} B^{\mu} \xi \psi_{\mu}+6 \mathrm{i} M \xi \sigma^{\mu} \bar{\psi}_{\mu}+16 \mathrm{i} \xi \lambda^{(R)}-3 M \bar{\xi} \overline{\mathcal{D}}\right) \mathcal{A} .
\end{aligned}
$$


Finally we compute $16 e \delta_{\text {susy }}\left(\psi_{\mu} \sigma^{\mu \nu} \psi_{\nu} \mathcal{A}\right)$ :

$$
\begin{aligned}
& 16 e \delta_{\text {susy }}\left(\psi_{\mu} \sigma^{\mu \nu} \psi_{\nu} \mathcal{A}\right) \\
&=32 e\left[\left(\delta_{\text {susy }} \psi_{\mu}\right) \sigma^{\mu \nu} \psi_{\nu}+\left(\delta_{\text {susy }} E_{a}^{\mu}\right) \psi_{\mu} \sigma^{a \nu} \psi_{\nu}+\frac{1}{2} \psi_{\mu} \sigma^{\mu \nu} \psi_{\nu} \bar{\xi} \overline{\mathcal{D}}\right] \mathcal{A} \\
&= e\left(32 \nabla_{\mu} \xi \sigma^{\mu \nu} \psi_{\nu}+24 \mathrm{i} B^{\mu} \xi \psi_{\mu}-6 \mathrm{i} M \psi_{\mu} \sigma^{\mu} \bar{\xi}\right. \\
&\left.+64 \mathrm{i} \psi_{\mu} \sigma^{\rho \nu} \psi_{\nu}\left(\psi_{\rho} \sigma^{\mu} \bar{\xi}-\xi \sigma^{\mu} \bar{\psi}_{\rho}\right)+16 \psi_{\mu} \sigma^{\mu \nu} \psi_{\nu} \bar{\xi} \overline{\mathcal{D}}\right) \mathcal{A} .
\end{aligned}
$$

Summing up (B.3), (B.6), (B.7), (B.9) and (B.10) one sees that indeed all terms cancel out except for terms containing $\nabla_{\mu}$ and terms at least quadratic in the gravitino. Playing

a bit with spinor indices and using (5.38), one can check that these therms combine to a total divergence:

$$
\delta_{\text {susy }} L_{\text {old }}=\partial_{\mu}\left(32 e \xi \sigma^{\mu \nu} \psi_{\nu} \mathcal{A}-4 \mathrm{i} e \xi \sigma^{\mu} \overline{\mathcal{D}} \mathcal{A}\right)+\text { c.c. }
$$

\section{References}

[1] B. de Wit and J. Louis, "Supersymmetry and dualities in various dimensions," in Strings, Branes and Dualities. L. Baulieu et al (eds), Kluwer, Dordrecht, The Netherlands, 1999, pp. 33-101. hep-th/9801132.

[2] A. Van Proeyen, "Tools for supersymmetry," hep-th/9910030.

[3] P. Van Nieuwenhuizen, "Supergravity," Phys. Rept. 68 (1981) 189-398.

[4] J. Wess and J. Bagger, Supersymmetry and Supergravity. Princeton Univ. Press, Princeton, NJ, 1992.

[5] S. J. Gates, M. T. Grisaru, M. Rocek, and W. Siegel, "Superspace, or one thousand and one lessons in supersymmetry," Front. Phys. 58 (1983) 1-548, hep-th/0108200.

[6] H. P. Nilles, "Supersymmetry, supergravity and particle physics," Phys. Rept. 110 (1984) 1.

[7] P. C. West, Introduction to Supersymmetry and Supergravity. World Scientific, Singapore, Singapore, 1990.

[8] N. Dragon, U. Ellwanger, and M. G. Schmidt, "Supersymmetry and supergravity," Prog. Part. Nucl. Phys. 18 (1987) 1-91.

[9] I. L. Buchbinder and S. M. Kuzenko, Ideas and Methods of Supersymmetry and Supergravity: Or a Walk Through Superspace. IOP, Bristol, UK, 1998.

[10] H. Goldschmidt, "Integrability criteria for systems of non-linear partial differential equations," J. Diff. Geom. 1 (1967) 269-307.

[11] F. J. Pommaret, Systems of Partial Differential Equations and Lie Pseudogroups. Gordan and Breach, New York, 1978. 
[12] P. J. Olver, Applications of Lie Groups to Differential Equations. Graduate Texts in Mathematics Vol. 107, Springer, Berlin, 1998.

[13] D. J. Saunders, The Geometry of Jet Bundles. Cambridge Univ. Press, Cambridge, 1989.

[14] I. M. Anderson, "The variational bicomplex," tech. rep., Utah State Univ., 1989, http://www.math.usu.edu/ fg_mp/pages/publications/publications.html.

[15] G. Barnich, F. Brandt, and M. Henneaux, "Local BRST cohomology in gauge theories," Phys. Rept. 338 (2000) 439-569, hep-th/0002245.

[16] E. Noether, "Invariante Variationsprobleme," Nachrichten Kgl. Ges. d. Wiss. z. Göttingen, Math.-phys. Kl. 2 (1918) 235-257.

[17] M. Henneaux and C. Teitelboim, Quantization of Gauge Systems. Princeton Univ. Press, Princeton, NJ, 1992.

[18] D. Z. Freedman, P. van Nieuwenhuizen, and S. Ferrara, "Progress toward a theory of supergravity," Phys. Rev. D13 (1976) 3214-3218.

[19] S. Deser and B. Zumino, "Consistent supergravity," Phys. Lett. B62 (1976) 335.

[20] K. S. Stelle and P. C. West, "Minimal auxiliary fields for supergravity," Phys. Lett. B74 (1978) 330.

[21] S. Ferrara and P. van Nieuwenhuizen, "The auxiliary fields of supergravity," Phys. Lett. B74 (1978) 333.

[22] F. Brandt, "Local BRST cohomology and covariance," Commun. Math. Phys. 190 (1997) 459-489, hep-th/9604025.

[23] F. Brandt, "Jet coordinates for local BRST cohomology," Lett. Math. Phys. 55 (2001) 149-159, math-ph/0103006.

[24] N. Dragon, "Torsion and curvature in extended supergravity," Z. Phys. C2 (1979) $29-32$.

[25] F. Brandt, "Lagrangean densities and anomalies in four-dimensional supersymmetric theories." Ph.D. thesis (in German), Hannover, 1991, http://home.nexgo.de/fbrandt/pub.html.

[26] R. Grimm, J. Wess, and B. Zumino, "A complete solution of the Bianchi identities in superspace," Nucl. Phys. B152 (1979) 255-265.

[27] F. Brandt, "Local BRST cohomology in minimal D=4, N=1 supergravity," Annals Phys. 259 (1997) 253-312, hep-th/9609192.

[28] D. Z. Freedman, "Supergravity with axial gauge invariance," Phys. Rev. D15 (1977) 1173-1174. 
[29] M. F. Sohnius and P. C. West, "An alternative minimal off-shell version of $\mathrm{N}=1$ supergravity," Phys. Lett. B105 (1981) 353-357. 